\author{
Department of Civil Engineering
}

Computational Continuum Mechanics Group

\title{
Phase Field Modeling of Dynamic Brittle Fracture at Finite Strains
}

A THESis SUBMitTED IN PARTIAL FULFILMENT OF THE REQUIREMENTS FOR A MASTER OF SCIENCES DEGREE IN THE FIELD OF CIVIL ENGINEERING

Prepared for: University of CAPE TOWN

Author:

Mr Emmanuel Omatuku NGONGO
Supervisor:

A/Prof Sebastian Skatulla 
The copyright of this thesis vests in the author. No quotation from it or information derived from it is to be published without full acknowledgement of the source. The thesis is to be used for private study or noncommercial research purposes only.

Published by the University of Cape Town (UCT) in terms of the non-exclusive license granted to UCT by the author. 


\section{Plagiarism declaration}

I, Emmanuel Omatuku Ngongo, declare that this thesis titled, "Phase Field Modeling of Dynamic Brittle Fracture at Finite Strains." and the work presented in this document are my own. I confirm that:

- This work was done in candidature for a Master of Sciences degree in the field of Civil Engineering at the University of Cape Town.

- I know the meaning of plagiarism and declare that all the work in the document, save for that which is properly acknowledged, is my own.

- This thesis has been submitted to the Turnitin module (or equivalent similarity and originality checking software) and I confirm that my supervisor has seen my report and any concerns revealed by such have been resolved with my supervisor.

- I have not allowed and will not allow anyone to copy this work with the intention of passing it as their own.

Signature: Signed by candidate Date: January 16, 2019 


\section{Abstract}

Fracture is the total or partial separation of an initially intact body through the propagation of one or several cracks. Computational methods for fracture mechanics are becoming increasingly important in dealing with the nucleation and propagation of these cracks. One method is the phase field approach, which approximates sharp crack discontinuities with a continuous scalar field, the so-called phase field. The latter represents the smooth transition between the intact and broken material phases. The evolution of the phase field due to external loads describes the fracture process. An original length scale is used to govern the diffusive approximation of sharp cracks. This method further employs a degradation function to account for the loss of the material stiffness during fracture by linking the phase field to the body's bulk energy. To prevent the development of unrealistic crack patterns and interpenetration of crack faces under compression, this study uses the anisotropic split of the bulk energy, as proposed by Amor et al. [5], to model the different fracture behavior in tension, shear and compression.

This research is part of a larger project aimed at the modeling of Antarctic sea ice dynamics. One aspect of this project is the modeling of the gradual break-up of the consolidated ice during spring. As a first step, this study reviews a phase field model used for dynamic brittle fracture at finite strains. Subsequently, this model is implemented into the in-house finite element software SESKA to solve the benchmark tension and shear tests on a single-edge notched block. The implementation adopts the so-called monolithic scheme, which computes the displacement and phase field solutions simultaneously, with a Newmark time integration scheme. The results of the solved problems demonstrate the capabilities of the implemented dynamic phase field model to capture the nucleation and propagation of cracks. They further confirm that the choice of length-scale and mesh size influences the solutions. In this regard, a small value of the length-scale converges to the sharp crack topology and yields a larger stress value. On the other hand, a large length-scale parameter combined with a too coarse mesh size can yield unrealistic results. 


\section{Acknowledgements}

Firstly, I would like to express my sincere gratitude to my research supervisor, A/Prof Sebastian Skatulla, for providing me with such an exciting topic. I am also grateful for his continuous support, guidance and knowledge.

Secondly, I would like to thank Prof Michael Kaliske (Technische Universität Dresden) and his students Christian SteINKE and Bo YIN for discussions and their insights on the phase field modeling of fracture.

Thirdly, I would like to thank my parents, my siblings and my partner Kim Deborah Thöni and for their love and endless support in my pursuit of this Masters degree. Next, I would like to thank my friends who gave me the right diversion from this work and different perspectives.

This research has been supported by the Centre for High Performance Computing South Africa and the National Research Foundation of South Africa (Grant Numbers 104839 and 105858). Opinions expressed and conclusions arrived at, are those of the author and are not necessarily to be attributed to the NRF. 


\section{Table of contents}

Plagiarism declaration $\quad$ i

Abstract $\quad$ ii

Acknowledgements $\quad$ iii

List of figures $\quad$ vi

List of tables $\quad$ vii

Notations $\quad$ viii

1 Introduction 1

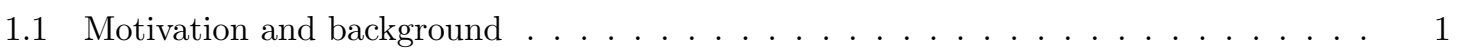

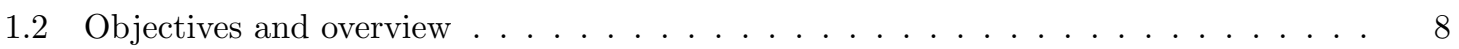

2 Classical continuum mechanics $\quad 10$

2.1 Kinematics . . . . . . . . . . . . . . . . . . . . . . . . . . . 10

2.2 Strain measures . . . . . . . . . . . . . . . . . . . . . . . . . . 13

2.3 Stress measures . . . . . . . . . . . . . . . . . . . . . . . . . . . . . . . 14

2.4 Balance laws . . . . . . . . . . . . . . . . . . . . . . . . . . . . 15

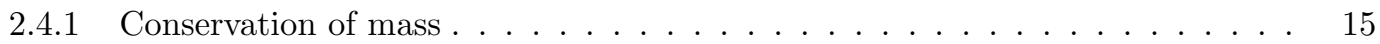

$2.4 .2 \quad$ Linear momentum principle . . . . . . . . . . . . . . . . . 16

2.4 .3 Angular momentum principle . . . . . . . . . . . . . . . . 18

2.5 Constitutive formulation of a neo-Hookean material . . . . . . . . . . . . . . 20

3 Fundamentals of fracture mechanics $\quad 22$

3.1 Basic definitions . . . . . . . . . . . . . . . . . . . . . . . . 22

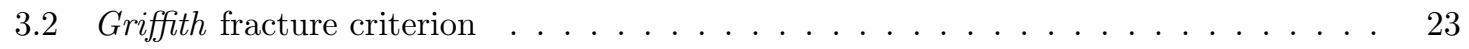

4 Phase field model of dynamic brittle fracture $\quad 26$

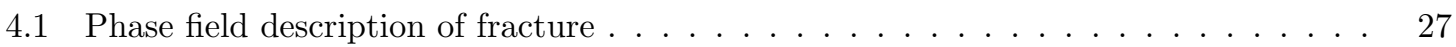

4.1.1 Motivation of the phase field approximation in one dimension . . . . . . . 27 
4.1.2 Motivation of the phase field approximation in two and three dimensions . . 29

4.2 Degradation of the body's bulk energy . . . . . . . . . . . . . . . . . . . . . 30

4.2 .1 Isotropic split . . . . . . . . . . . . . . . . . . . 30

4.2 .2 Anisotropic split . . . . . . . . . . . . . . . . . . . 32

4.3 Irreversibility of the fracture process . . . . . . . . . . . . . . . . . . . . . 36

4.4 Variational formulation for dynamic fracture . . . . . . . . . . . . . . . . 38

5 Finite element implementation $\quad 40$

5.1 Weak forms and spatial discretization . . . . . . . . . . . . . . . . . . . . 41

5.2 Monolithic temporal discretization and iterative solution . . . . . . . . . . . . . . 43

$\begin{array}{lll}6 & \text { Numerical examples } & 47\end{array}$

6.1 Single-edge notched block under tension . . . . . . . . . . . . . . . . . . . . . . . 48

6.2 Single-edge notched block under shear . . . . . . . . . . . . . . . . . 55

7 Conclusions and future work $\quad 60$

7.1 Conclusions . . . . . . . . . . . . . . . . . . . . . . . . 60

7.2 Future work .............................. 62

7.2.1 Extension of the implemented phase field model for dynamic fracture . . . . 62

7.2.2 Application of the phase field model to the fracture of Antarctic ice . . . . . 64

$\begin{array}{ll}\text { Appendix } & 65\end{array}$

$\begin{array}{lr}\text { A SESKA } & 66\end{array}$

$\begin{array}{lr}\text { B Ethics approval } & 67\end{array}$

$\begin{array}{lr}\text { Bibliography } & 69\end{array}$ 


\section{List of Figures}

2.1 Configuration and motion of a continuum body. . . . . . . . . . . . . . . 11

3.1 Cracked body. . . . . . . . . . . . . . . . . . . . . . . 22

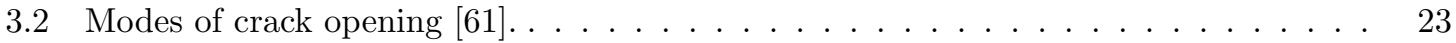

4.1 Sharp and diffusive crack modeling. (a) Sharp crack at $x=0$ and (b) diffusive crack at $x=0$ modeled with the length-scale $l \ldots \ldots \ldots \ldots \ldots 27$

4.2 (a) Body with internal discontinuities (sharp cracks) $\Gamma$ and (b) Approximation of internal discontinuities using the phase field $d(\boldsymbol{X}, t) \ldots \ldots \ldots \ldots . \ldots . . \ldots 29$

6.1 Problem definition of a single-edge notched block under tension. . . . . . . . . . . 49

6.2 Generated mesh of single-edge notched block under tension. . . . . . . . . . . . . 50

6.3 (a) Boundary conditions for tension case used in Hesch et al. [45]. (b) Phase field contour plot for tension case obtained by Hesch et al. [45] . . . . . . . . . . . . . . 51

6.4 Phase field contour plot for tension case 1 at $l=0.05 \mathrm{~m} \ldots \ldots \ldots \ldots \ldots$. . . . . 51

6.5 Phase field contour plot for tension case 2 at $l=0.03 \mathrm{~m} \ldots \ldots \ldots \ldots \ldots 2$

6.6 Phase field contour plot for tension case 3 at $l=0.02 \mathrm{~m} \ldots \ldots \ldots \ldots \ldots$

6.7 Stress $\boldsymbol{\sigma}_{y y}$ vs. Almansi strain $\boldsymbol{e}_{y y}$ at notch point for cases of block under tension. . . 53

6.8 Stress $\boldsymbol{\sigma}_{y y}$ vs. Length-scale $l$ at notch point for cases of block under tension. . . . . 54

6.9 Geometry and boundary conditions of single-edge notched block under shear. . . . . 55

6.10 Generated mesh of single-edge notched block under shear. . . . . . . . . . . . . . 56

6.11 (a) Boundary conditions for shear case used in Hesch et al. [45]. (b) Phase field contour plot for shear case obtained by Hesch et al. [45] . . . . . . . . . . . . . . 57

6.12 Phase field contour plot for shear case 1 at $l=0.10 \mathrm{~m} \ldots \ldots \ldots \ldots \ldots$

6.13 Phase field contour plot for shear case 2 at $l=0.05 \mathrm{~m} \ldots \ldots \ldots \ldots$. . . . . . 58

6.14 Phase field contour plot for shear case 3 at $l=0.02 \mathrm{~m} \ldots \ldots \ldots \ldots \ldots$ 


\section{List of Tables}

6.1 Model parameters for the single-edge notched block under tension. . . . . . . . . . 49

6.2 Study parameters for single-edge notched block under tension. . . . . . . . . . . 50

6.3 Study parameters for single-edge notched block under shear. . . . . . . . . . . . . 56 


\section{Notations}

This thesis uses several variables. For convenience, the variables that are frequently used herein are provided below.

$t \quad$ Specific point in time, see equation (2.1) on page 11

$\boldsymbol{F} \quad$ Deformation gradient, see equation (2.3) on page 11

$\boldsymbol{X} \quad$ Referential position vector, see equation (2.1) on page 11

$\boldsymbol{x} \quad$ Current position vector, see equation (2.1) on page 11

$\mathcal{B} \quad$ Material body, see equation (2.1) on page 11

$\mathcal{B}_{t} \quad$ Material body at a given time $t$, see equation (2.1) on page 11

$\boldsymbol{u} \quad$ Displacement vector field, see equation (2.2) on page 11

$J \quad$ Jacobian, see equation (2.5) on page 12

$\boldsymbol{n} \quad$ Normal vector in the current configuration, see equation (2.7) on page 12

$\boldsymbol{N} \quad$ Normal vector in the reference configuration, see equation (2.7) on page 12

$\mathrm{d} a \quad$ Small area in the current configuration, see equation (2.7) on page 12

$\mathrm{d} A \quad$ Small area in the reference configuration, see equation (2.7) on page 12

$\mathrm{d} \boldsymbol{x} \quad$ Line elements in the current configuration, see equation (2.4) on page 12

$\mathrm{d} \boldsymbol{X} \quad$ Line elements in the reference configuration, see equation (2.4) on page 12

C Right Cauchy-Green strain tensor, see equation (2.11) on page 13

$\boldsymbol{E} \quad$ Green strain tensor, see equation (2.13) on page 13

$\boldsymbol{b} \quad$ Body forces, see equation (2.17) on page 14

$\boldsymbol{t}^{(\boldsymbol{\nu})} \quad$ Surface forces, see equation (2.17) on page 14

$\boldsymbol{\sigma} \quad$ Cauchy stress tensor, see equation (2.18) on page 14

$\boldsymbol{P} \quad$ First Piola-Kirchhoff stress tensor, see equation (2.19) on page 14

$\boldsymbol{S} \quad$ Second Piola-Kirchhoff stress tensor, see equations (2.20, 2.58 and 4.24) on pages 14, 21 and 34

$m \quad$ Mass of a material continuum body, see equation (2.23) on page 15

$\rho \quad$ Mass density, see equation (2.23) on page 15

$\dot{\boldsymbol{x}} \quad$ Velocity field, see equation (2.32) on page 16

$\ddot{\boldsymbol{x}} \quad$ Acceleration field, see equation (2.33) on page 17

$W \quad$ Free-energy function, see equation (2.57) on page 20

$U(J) \quad$ Volumetric part of energy function, see equation (2.54) on page 20

$\tilde{W}(\boldsymbol{C})$ Deviatoric part of energy function, see equation (2.55) on page 20

$\tilde{\boldsymbol{C}} \quad$ Deviatoric part of right Cauchy-Green strain tensor, see equation (2.56) on page 20 


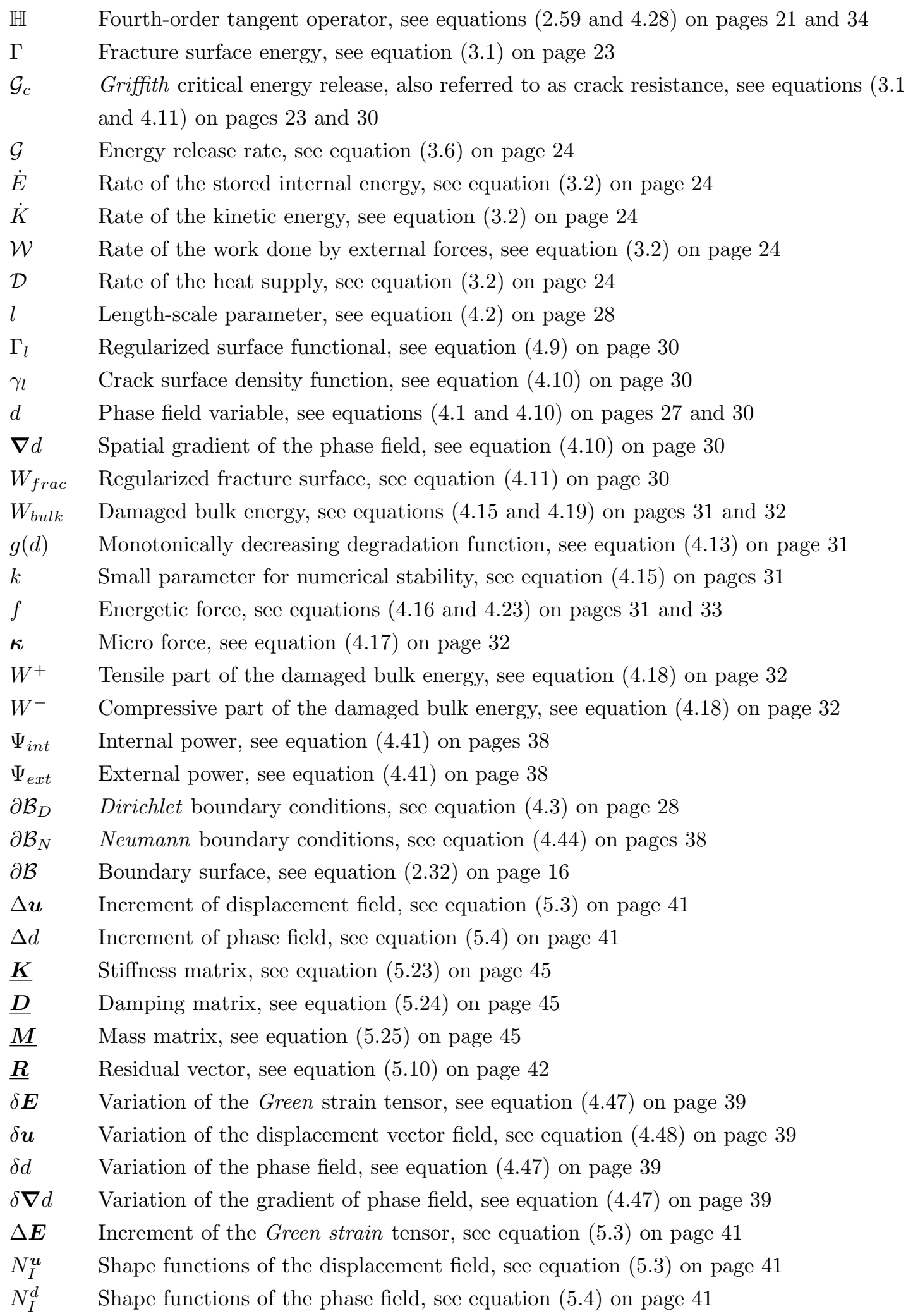




\section{Chapter 1}

\section{Introduction}

\subsection{Motivation and background}

Fracture is a process which takes place in engineering and natural structures. This process consists of the total or partial separation of an initially intact structure through the propagation of one or several cracks. In engineering design, this process is a significant concern, because it weakens the integrity of structural components and causes structural failure [40]. In natural structures such as the Antarctic sea ice cover or the continental ice shelf, fracture is of vital importance to develop accurate predictive models of the climate system and met-ocean thermodynamics [95] [27]. Fracture of Antarctic sea ice is particularly associated with this work. The latter forms part of a multidisciplinary project which aims at the modeling of Antarctic sea ice dynamics. An aspect of this project is the modeling of Antarctic sea ice fracture. This work constitutes the first step in addressing this aspect.

Antarctic sea ice, especially at its largest extent in winter, behaves as an almost impermeable blanket that shields the underlying cold Southern ocean from the atmospheric influence. Thus, sea ice prevents the transfer of wind and wave energy, as well as constituents such as atmospheric gas, dust and aerosols [114]. Furthermore, the interaction between these constituents affects the physical and mechanical properties of sea ice and thus governs the response of the ice to anthropogenic warming. Moreover, the anticipated response of sea ice to this type of warming has recently become a major concern since the extent of Antarctic sea ice reached in the spring of 2017 its lowest minima in existing records. The gradual fracture of sea ice in spring renders this blanket increasingly 
permeable through the associated crack openings and leads, and influences sea ice dynamics in the region. Hence, the understanding and analysis of Antarctic sea ice fracture are essential to describe physical phenomena of the metocean environment in the Southern Ocean.

The need to understand and analyze fracture has led to the use of experimental tests and the development of numerical simulations. However, experimental tests present some limitations because they are time-consuming and rather expensive. Furthermore, they may not be practical during engineering design stages and on natural structures. Therefore, numerical simulations are commonly employed to circumvent these limitations. As a result, substantial research has been conducted to develop reliable fracture models and numerical implementations. The primary aim of these models is to track the evolution of the fracture process due to external loads by predicting the geometry of the crack path. The latter includes the kinking of cracks and their bifurcations into several cracks. To this end, these models rely on the fracture criteria used in the field of fracture mechanics to predict the evolution of pre-existing cracks, the initiation and growth of new cracks as well as the velocity of the crack propagation [61].

The pioneering theories of modern fracture mechanics date back to the concept presented by Griffith [39] and later extended by Irwin [51]. Griffith regarded cracks as the macroscopic indication of debonding processes at the crystalline level. He further proposed that the energy density at each point of the crack surface can accurately describe the debonding process, and that crack propagation stems from the competition between the body's bulk energy and fracture energy. Irwin further extended Griffith's work by redefining the fracture energy density and describing cracks with respect to the singular stresses found at crack tips. He also demonstrated that his concept is equivalent to Griffith's energetic approach. These pioneering works made it possible to evaluate cracks using classical continuum mechanics and allow to explore various research avenues in theoretical fracture mechanics [61]. However, Griffith's theory is limited in the sense that it does not address the process of crack nucleation and does not predict the crack path.

In addressing the shortcomings of Griffith's fracture criterion, several approaches have been proposed. One approach is the use of a deflection criterion which predicts the crack path under mixed loading. Several authors investigated this criterion with a broad variety of techniques. For example, Erdogan and Sih [30] introduced the maximum hoop stress criterion which proposes that crack propagates in the direction of maximum circumferential stresses. Wu [119] applied the maximum energy release rate criterion, and Gol'dstein and Salganik [38] adopted the principle of local symmetry as a propagation criterion. Chambolle et al. [22] revisited in a two-dimensional setting the notion of 
energy release rate. Another approach is the criterion that Li and Zhang [70] proposed to establish a failure criterion for arbitrary stress concentrations. They developed this criterion based on the self-consistent fracture criterion proposed by Leguillon [69] and combined the strength criterion with the incremental Griffith criterion. In this approach, a critical stress value and the crack resistance are the parameters that govern the growth and nucleation of cracks. Therefore, the singularity at a crack tip ensures that the strength criterion holds at that tip. This limiting case agrees with Griffith's differential formulation. However, for the case of weaker singularities, which arise at notches, the characteristic length of the nucleated crack results from the combination of the critical stress and crack resistance with the sample geometry and loading.

In contrast to the approaches outlined above, Francfort and Marigo [33] did not attempt to remedy the weakness of the Griffith criterion with additional criteria. Instead, they proposed a variational reformulation of the classical Griffith criterion. The formulation addresses the issues of crack initiation, crack path and smoothness of the crack evolution. Its driving principle is the global energy minimization. This energy includes the body's bulk energy, Griffith's surface energy and the potential energy due to external forces. A benefit of this variational formulation is to mainly be consistent with Griffith's theory, departing as little as possible to handle the nucleation and branching of cracks as well as the interaction between multiple cracks. However, this formulation leads to an increase in computational cost due to the complexity of a direct implementation of sharp discontinuities, particularly in the modeling of complex crack topologies. Consequently, this drawback led to the use and development of several computational methods for the implementation of this formulation. Some of these methods are the finite element method (FEM) proposed in e.g. [50] [74] [123], remeshing techniques presented in e.g. [23] [41] [60], the extended finite element method (X-FEM) introduced in e.g. [8] [82] [83] [84] and the phase field method developed in e.g. [77] [78, 79] [101] [110]. FEM, which is the most commonly used method, discretizes a continuous body into an equivalent system of finite elements with a specific number of nodal points. Rather than requiring the analytical solution of partial differential equations for unknown field variables, the formulation of FEM results in a system of simultaneous algebraic equations for the solution. Thus, FEM yields approximate values of the unknown field variables at discrete numbers of points in the continuum by using continuous shape functions between the nodes [74]. This characteristic limits the use of conventional FEM in dealing with field discontinuities expected at cracks in fracture problems.

The common implementation techniques to overcome the limitation of FEM and subsequently model discontinuities are interface element formulations and strategies of elemental and nodal enrichment. 
The basic idea of these techniques is to update the mesh at every incremental macro-crack step such that the crack path remains independent of the initial mesh [60]. Methods using adaptive interface elements are outlined in Xu and Needleman [120], Camacho and Ortiz [20], Pandolfi and Ortiz [90], Gürses and Miehe [41] and Miehe and Gürses [76]. Another technique is the X-FEM method, that Moës et al. [83] proposed. This method enriches the standard approximation of the displacement near a crack with the addition of discontinuous and near-tip asymptotic fields by adopting the partition of unity method presented by Melenk and Babuška [75]. This technique allows for the simulation of crack growth without remeshing. However, its application in a three-dimensional setting is rather challenging, especially when dealing with intricate crack patterns [61]. For further insight in the X-FEM method, the reader is referred to e.g. Belytschko et al. [9], Fagerström and Larsson [31], Song et al. [107], Song et al. [108] and Song and Belytschko [106]. An alternative approach is the finite elements with embedded discontinuities which models the branching of cracks using a branching criterion based on velocity. This approach is proposed by Armero and Linder [7] and Linder and Armero [71].

In contrast to the methods outlined above, the phase field method uses a conceptually different approach and addresses the difficulty that the X-FEM method faces in three-dimensional fracture problems. This method rests on the construction of a Cahn-Hilliard [19], Ginzburg-Landau [37] or entropy functional. Initial phase field models addressed the dendritic solidification processes of pure and binary materials. The models of Fix [32] and Langer [66] were first to describe first-order phase transitions, while Collins and Levine [24] independently proposed a similar diffusive interface model. For further information on early phase field models, see Gaginalp et al. [34], Penrose and Fife [91, 92], Caginalp and Socolovsky [18], Kobayashi [56, 57, 58] and Caginalp and Jones [17]. The basic concept of the phase field method is to add a continuous scalar field, also referred to as the phase field or order parameter, which characterizes the degree of crystallinity or atomic order or disorder in a phase. The popularity of this method is based on its ability to simulate complex free boundary problems elegantly. Some of these problems include dendritic growth (e.g. Karma and Rappel [53]), solidification of binary alloys (e.g. Echebarria et al. [29]), polycrystals formation in a three-dimensional setting (e.g. Kobayashi and Warren [59]) and ferroelectric phase transitions (e.g. Schrade et al. [103] and Müller et al. [85]).

To model fracture, the phase field method assigns a value of zero to the phase field to indicate an undamaged material and a value of one for a broken material. This field circumvents the discontinuous jumps at crack interfaces by interpolating between the undamaged and broken material phases 
across a diffusive interface. An original length scale parameter governs the width of this interface. Thus, the underlying sharp crack interface is recovered when this parameter is infinitesimally small. The evolution of the phase field is represented by partial differential equations, which describe the fracture process due to external loads. Furthermore, a degradation function models the change in the material stiffness between the undamaged and broken states through the coupling of the phase field and displacement field. Phase field models for fracture present several advantages over sharp crack models. Firstly, they do not consider jump discontinuities. Consequently, their implementation within a finite element formulation is possible with standard continuous shape functions. Secondly, the simulation of crack growth does not require the use of remeshing techniques. Hence, phase field models can accurately describe complex topological changes, including the multiple branching or merging of cracks [79]. Finally, the coupled equations automatically satisfy the boundary conditions at phase interfaces. Thus, these models can circumvent the explicit treatment of these conditions $[2]$.

The development of phase field methods for fracture problems occurred independently within the physics' and mechanics' communities. As a result, these communities adopted substantially different concepts and techniques to derive the constitutive and equation of the phase field evolution. The physics' community proposed models for fracture problems by adopting the Landau-Ginzburg phase transition [65]. Some of these models are presented in e.g. Aranson et al. [6], Karma et al. [54], Eastgate et al. [28], Henry and Levine [43], Karma and Lobkovsky [52], Hakim and Karma [42], Corson et al. [25] and Spatschek et al. [109]. However, these models are not related to Griffith's theory as they do not incorporate the Griffith-type critical energy release rate in their formulations. Furthermore, the direct formulation of these models in a dynamic setting further makes it difficult to compare their results with engineering benchmark problems [78].

On the other hand, the mechanics' community developed models based on the variational formulation of quasi-static brittle fracture proposed by Francfort and Marigo [33]. Bourdin et al. [14] introduced a regularized version of this formulation to handle the minimization requirement for quasi-static problems. This regularization results in an energy functional, which is very similar to the socalled Mumford-Shah potential commonly employed in image segmentation [86]. Ambrosio and Tortorelli [4] were first to introduce a phase field approximation of this potential. Bourdin et al. [14] then adopted this approximation to facilitate the numerical solution of the variational formulation. The virtue of this approach is that the fracture problem is reformulated into a system of coupled differential equations to determine the nucleation, propagation or bifurcation of cracks with no ad- 
hoc conditions. Giacomini [36] also successfully applied the approximation introduced by Ambrosio and Tortorelli [4] to the quasi-static evolution of brittle fracture in linear elastic bodies. Several authors, e.g. Larsen [67], Larsen et al. [68], Bourdin et al. [16], Borden et al. [12] and Schlüter et al. [101], have extended the early quasi-static regularized models to dynamic fracture. However, some of these models do not consider applications to structural engineering [78].

As an alternative to the phase field models mentioned above, Miehe et al. [78, 79] introduced a different quasi-static formulation of the phase field approximation for applications to engineering problems. The approach of Miehe et al. [78, 79] relies on continuum mechanics and thermodynamic principles. They motivated that the rate-independent diffusive formulation of Bourdin et al. [14] can only use a time-discrete model to capture the irreversibility of the fracture process. In achieving this goal, the diffusive formulation imposes hard Dirichlet boundary conditions on the phase field. They further argued that the phase field models above are not physically realistic for most problems as these models assume that the energy release drives the fracture process in both tension and compression. This drawback confines the application of these models to boundary value problems under tension. Furthermore, most of these models are not thermodynamically consistent, and their use is only limited to the monotonous loading regime of arbitrary sub-domains of a solid during fracture.

Most of the phase field models for fracture, including those mentioned above, have been developed to deal with quasi-static problems at small strains. Beyond this type of problems, phase field fracture models can also address dynamic problems, finite strain problems, cohesive fracture, conchoidal fracture, fracture in biological tissues and ductile fracture. On dynamic brittle fracture, several authors have investigated this problem at small strains. To name a few, Hofacker and Miehe $[47,48]$ extended the quasi-static formulation of Miehe et al. [78, 79] to a dynamic case. Similarly, Schlüter et al. [101] extended the quasi-static model of Kuhn and Müller [62] to account for dynamic effects. Carlsson and Isaksson [21] modeled the dynamic crack propagation in wood fiber composites analyzed by high-speed photography. For further insight into dynamic brittle fracture models, see Borden et al. [12] and Liu et al. [73].

With regards to the modeling of fracture at finite strains, Hesch and Weinberg [44] proposed a model for a general nonlinear material and finite deformations. They used a multiplicative split of the principal stretches to account for the different behavior of fracture in tension, shear and compression. Hesch et al. [45] further extended this model with a polyconvex formulation that guarantees numerical stability for the full range of deformations and arbitrary hyperelastic materials. 
Miehe and Schänzel [77] modeled fracture in a rubbery polymer which may exhibit a very complicated inelastic behavior at finite strains. Reinoso et al. [98] presented a model of brittle fracture for the large deformation analysis of thin-walled structures using an enhanced strain-based formulation. The fracture model of Teichtmeister et al. [113] accounted for anisotropic material behavior at small and finite strains. On the other hand, Weinberg and Hesch [117] combined a second-order and fourth-order variational formulations of the phase field with a finite deformation ansatz for a general nonlinear material.

In addressing cohesive fracture, Verhoosel and de Borst [116] developed a model using a cohesive zone approach in an energetic framework suitable for the incorporation of a phase field approach. To model conchoidal fracture problems, Thomas [115] presented a model for anisotropic fracture at finite strains. This model rests on a modification of the Ambrosio-Tortorelli model. The latter is formulated for polyconvex energy densities in terms of the modified invariants of the right CauchyGreen strain tensor and is also augmented by a viscous dissipation for the phase field variable. For the modeling of failure phenomena in soft biological tissues at finite strains, Raina and Miehe [94] presented a phase field model equipped with an anisotropic crack driving force. Several publications have addressed ductile fracture at finite strains. Ambati et al. [3] extended a ductile fracture previously proposed at small strains to a three-dimensional finite strain setting. Miehe et al. [80, 81] provided a rigorous variational-based framework and numerical implementation for the modeling of ductile fracture in elastic-plastic solids undergoing large strains.

Despite the advantages that the phase field method provides, some drawbacks still need to be addressed. Firstly, the phase field formulation is computationally demanding because this method primarily requires extremely fine meshes, at least defined locally in the area of expected crack propagation, and needs to resolve a small regularization length-scale. At present, phase field models are typically computed on fine non-adaptive meshes, mostly due to the lack of an efficient fully adaptive mesh refinement strategy. Secondly, accounting for the different fracture behavior in tension, shear and compression requires a specific split of the bulk energy. Two split methods are commonly used: The spectral split of Miehe et al. [78, 79] and the volumetric-deviatoric split of Amor et al. [5]. However, the split results in a strongly non-linear constitutive relation to be solved iteratively, which makes the corresponding formulation computationally expensive [2].

Furthermore, recent publications have shown the shortcomings of the energy split methods. Strobl and Seelig [111] reported that both splits might lead to the violation of basic crack boundary conditions as they do not account for the crack orientation. Thus, they presented the inclusion of 
information about the crack orientation into the phase field model. This remedy addresses the shortcoming of the common split methods in initially cracked specimens with a simple profile. However, it leads to unrealistic crack orientation at the crack tip and within fully degraded elements. Furthermore, the authors did not provide a procedure to obtain the crack evolution. Similarly to Strobl and Seelig [111], Steinke and Kaliske [110] developed a method, which rests on the information of the crack orientation. They introduced a directional split method which decomposes the strains and stresses with respect to a local crack coordinate system into normal and shear components. The latter are classified into crack driving and persistent components. Unlike Strobl and Seelig [111], the authors presented a procedure to obtain the crack evolution.

\subsection{Objectives and overview}

This research is part of a project ultimately aimed at the modeling of Antarctic sea ice fracture dynamics. One aspect of the project is the modeling of the gradual break-up of the consolidated ice during spring. As a first step in addressing this aspect, this thesis focuses on the phase field modeling of dynamic brittle fracture at finite strains. In this regard, this study adopts the phase field models proposed by Miehe and Schänzel [77] and Hofacker and Miehe [47, 48]. The primary objective of this study is to implement this model using a neo-Hookean material constitutive formulation and to investigate the effect of the length-scale parameter and the mesh dependency on the solutions. To this end, the phase field model is implemented within the in-house finite element framework SESKA. The outline of this thesis along with the goals of its chapters can be listed as follows:

Chapter 2 reviews the fundamentals of classical continuum mechanics, which are relevant to this study. In this regard, this chapter discusses the kinematic measures that describe the deformation and the stress and strain measures that relate forces to deformation. The fundamental physical laws that can describe the behavior of a body are also outlined. Furthermore, the constitutive law of a neo-Hookean material is introduced to describe the relationship between stresses within this material to specified strain measures.

Chapter 3 outlines the essential concepts of fracture mechanics by providing a macroscopic definition of a crack and the modes of crack opening. This chapter further discusses the Griffith fracture criterion, which defines a threshold for the initiation and propagation of the fracture process.

Chapter 4 discusses the phase field model for dynamic brittle fracture, following the works of Miehe 
and Schänzel [77] and Hofacker and Miehe [47, 48]. On this subject, a motivation of the phase field approximation of sharp cracks is provided. This chapter further reviews the degradation of the body's bulk energy under fracture. In this regard, the so-called isotropic and anisotropic split methods are reviewed. The latter method accounts for the different fracture behavior in tension and compression, while the former does not. In this chapter, the anisotropic split proposed by Amor et al. [5] is outlined. The irreversibility of the fracture process is further discussed and enforced with the history-field functional presented by Miehe et al. [78, 79]. Alternative enforcement methods are also briefly outlined. Finally, the variational formulation of this dynamic model is provided with the help of the neo-Hookean constitutive formulation provided in Chapter 2.

Chapter 5 presents the procedures for the implementation of the dynamic phase field model in the inhouse software SESKA. In this chapter, the weak forms of the equation of motion and the evolution equation of the phase field are provided. Then, the global discrete coupled system of equations is defined. Furthermore, this chapter explicitly provides the nodal residuals, tangent matrix, damping matrix, and mass matrix.

Chapter 6 investigates the capabilities of the implemented dynamic model by solving three-dimensional representative problems while using the anisotropic split. The problems include a single-edged notched block under tension and shear. This chapter further investigates the effect of the lengthscale and mesh size on the solutions.

Chapter 7 concludes the thesis and gives the scope for future studies. 


\section{Chapter 2}

\section{Classical continuum mechanics}

Continuum mechanics is a useful tool that can successfully describe various physical phenomena based on the macroscopic studies of a system without knowing the complexity of their internal structures. In this regard, this chapter briefly introduces the subject of continuum mechanics by covering fundamental equations that are relevant to this study. The main concepts presented herein are kinematics, stress and strain measures, balance laws and the constitutive formulation of a neoHookean material. For further details, the reader is referred to the comprehensive books of Holzapfel [49], Bonet and Wood [10], Tadmor et al. [112], Reddy [96] and Zienkiewicz et al. [123].

\section{$2.1 \quad$ Kinematics}

In continuum mechanics, a material body $\mathcal{B}$ is considered to have a continuous (or at least a piecewise continuous) distribution of matter in space and time. The body occupies geometrical regions denoted by $\mathcal{B}_{0}, \ldots, \mathcal{B}_{t}$ at a given time $t$, also known as configurations of $\mathcal{B}$ at time $t$. The regions are determined uniquely at any instant of time with the spatial vector $\boldsymbol{x}$, which identifies the material points $P \in \mathcal{B}_{t}$. The region is referred to as the reference (or undeformed) configuration of the body $\mathcal{B}_{0}$ at initial time $t_{0}=0$ and is associated with the referential position vector $\boldsymbol{X}$ with its Cartesian coordinates $X_{i}$. Herein, and in what follows, Latin indices take the values $i=1,2,3$. 


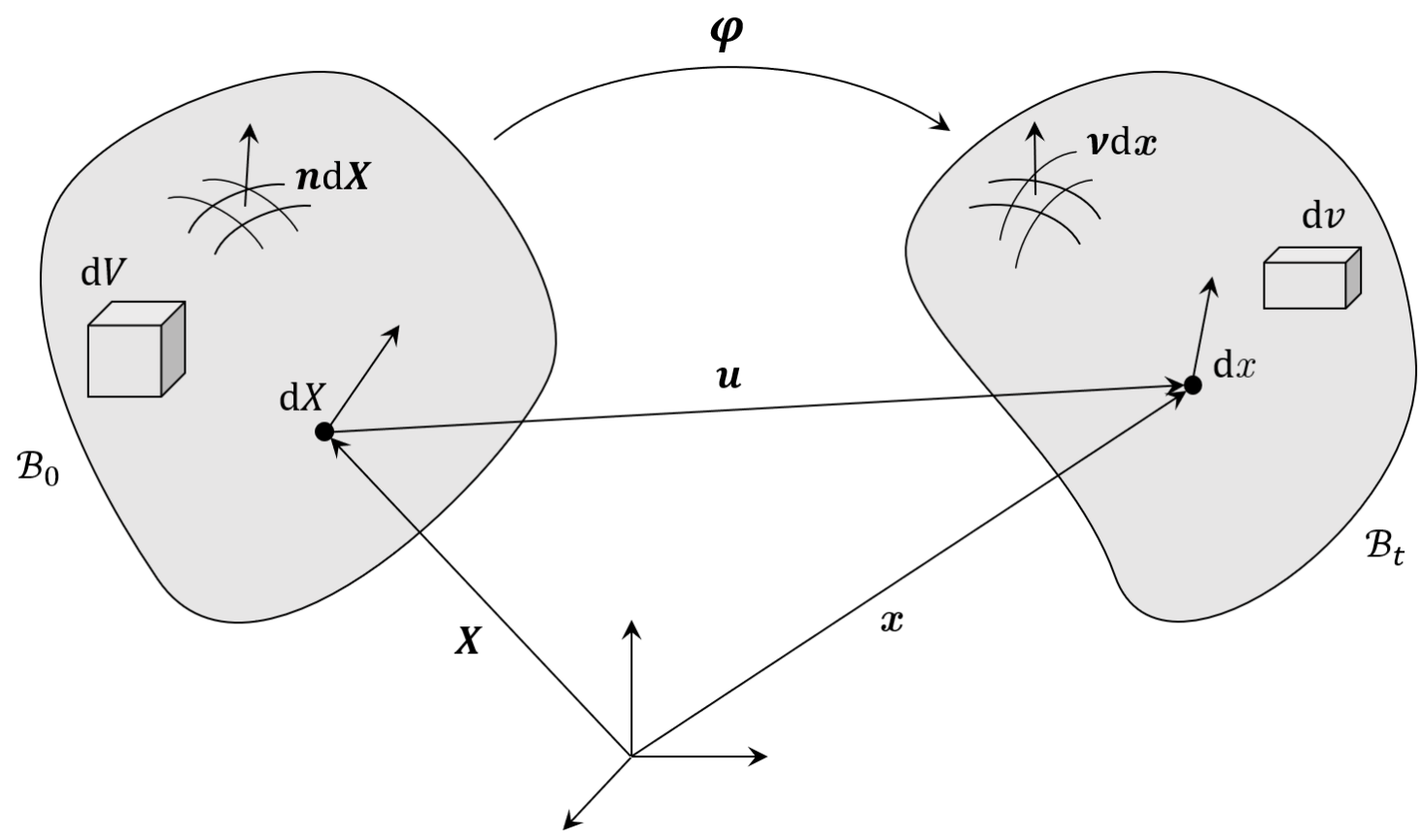

Figure 2.1: Configuration and motion of a continuum body.

The position vector $\boldsymbol{x}$ at the current time $t$ is related to the position vector $\boldsymbol{X}$ in the reference configuration $\mathcal{B}_{0}$ through the deformation mapping $\varphi$,

$$
\boldsymbol{x}=\varphi(X, t) .
$$

The displacement vector field $\boldsymbol{u}$, as shown in Figure 2.1, is defined as

$$
\boldsymbol{u}(\boldsymbol{X}, t)=\boldsymbol{x}-\boldsymbol{X}
$$

Thus, the current position $\boldsymbol{x}$ of a particle at time $t$ results from its referential position $\boldsymbol{X}$ plus its displacements $\boldsymbol{u}(\boldsymbol{X}, t)$ from that position. An essential measure of the deformation, hence the strain, caused by the mapping $\varphi$ is the deformation gradient defined as

$$
\boldsymbol{F}=\operatorname{Grad} \boldsymbol{X}
$$

where $\operatorname{Grad} \boldsymbol{X}:=\frac{\partial}{\partial \boldsymbol{X}}$ and its equivalent operation in the current configuration is given by $\operatorname{grad} \boldsymbol{x}:=$ $\frac{\partial}{\partial \boldsymbol{x}}$. This measure enables to characterize the behavior of motion in the vicinity of a point.

The linear transformation, which generates the line elements $\mathrm{d} \boldsymbol{x}$ in the current configuration through 
the action of the deformation gradient $\boldsymbol{F}$ on line elements $\mathrm{d} \boldsymbol{X}$, is given by

$$
\mathrm{d} \boldsymbol{x}=\boldsymbol{F} \mathrm{d} \boldsymbol{X} .
$$

The determinant of the deformation gradient $\boldsymbol{F}$, also known as the Jacobian, is denoted by

$$
J=\operatorname{det} \boldsymbol{F} .
$$

The change in volume between the reference and current configurations at time $t$ is defined by

$$
\mathrm{d} v=J \mathrm{~d} V
$$

where $\mathrm{d} v$ and $\mathrm{d} V$ denote the infinitesimal volume elements in the current and reference configurations, respectively. The assumption that $\boldsymbol{F}$ is invertible results in $J \neq 0$. The impermeability of matter, which means that volume elements cannot have negative volumes, confirms that a negative Jacobian is mathematically impossible. Hence the condition that the Jacobian must be strictly positive must hold for all $\boldsymbol{X} \in \mathcal{B}_{0}$ and all times $t$. The relation between the vector elements of the infinitesimally small areas $\mathrm{d} a$ and $\mathrm{d} A$, respectively defined in the current and reference configurations, is given by the Nanson formula

$$
\boldsymbol{\nu} \mathrm{d} a=\operatorname{det}(\boldsymbol{F}) \boldsymbol{F}^{-T} \boldsymbol{n} \mathrm{d} A=J \boldsymbol{F}^{-T} \boldsymbol{n} \mathrm{d} A .
$$

Herein $\boldsymbol{\nu}$ and $\boldsymbol{n}$ are respectively the normal vectors to the surface elements $\mathrm{d} a$ and $\mathrm{d} A$.

The local rate of change, which is defined as any change in time, is expressed as

$$
\frac{\partial(\bullet)}{\partial t}
$$

and the overall time rate of change of a group of particles is expressed by

$$
\frac{D}{D t}(\bullet)=\frac{\partial}{\partial t}(\bullet)+\frac{\partial}{\partial x_{i}}(\bullet) \frac{d x_{i}}{d t} .
$$

The material derivative of the Jacobian $J$ is given by

$$
\dot{J}=J \operatorname{div} \dot{\boldsymbol{x}},
$$


where "div" denotes the divergence operation in the current configuration.

\section{$2.2 \quad$ Strain measures}

Using the deformation gradient, the symmetric right Cauchy-Green strain tensor $\boldsymbol{C}$ is defined as

$$
\boldsymbol{C}=\boldsymbol{F}^{T} \boldsymbol{F},
$$

and the left Cauchy-Green strain tensor $\boldsymbol{b}$ is given by

$$
\boldsymbol{b}=\boldsymbol{F} \boldsymbol{F}^{T} \text {. }
$$

In turn, the symmetric Green strain tensor $\boldsymbol{E}$ can be expressed as a function of $\boldsymbol{C}$ using

$$
\boldsymbol{E}=\frac{1}{2}(\boldsymbol{C}-\mathbf{1}),
$$

while the Euler-Almansi strain tensor $\boldsymbol{e}$ is defined as function of $\boldsymbol{b}$ using

$$
e=\frac{1}{2}\left(\mathbf{1}-b^{-1}\right) .
$$

The stretch tensor and the right Cauchy-Green strain tensor are closely related through the following definition

$$
\boldsymbol{C}=\boldsymbol{U}^{2} .
$$

The rates of the right Cauchy-Green and Green strain tensors are expressed as

$$
\dot{\boldsymbol{C}}=2 \boldsymbol{F}^{T} \boldsymbol{d} \boldsymbol{F} \quad \text { and } \quad \dot{\boldsymbol{E}}=\boldsymbol{F}^{T} \boldsymbol{d} \boldsymbol{F},
$$

respectively. 


\subsection{Stress measures}

In continuum mechanics, stress measures are used to express the distribution of the force intensity for a bounded body in the deformed configuration $\mathcal{B}_{t}$. The stress in a body corresponds to externally applied body forces $\boldsymbol{b}$ and surface forces $\boldsymbol{t}^{(\boldsymbol{\nu})}$. The latter denotes the so-called traction vector acting on a surface with its unit normal vector $\boldsymbol{\nu}$. The Cauchy stress principle states that the resulting force $\Delta f$ on a surface element $\Delta a$ which is part of a cutting plane throughout the body $\mathcal{B}_{t}$ defines the traction vector $\boldsymbol{t}^{(\boldsymbol{\nu})}$ as follows

$$
\lim _{\Delta a \rightarrow 0} \frac{\Delta \boldsymbol{f}}{\Delta a}=\frac{\mathrm{d} \boldsymbol{f}}{\mathrm{d} a}=\boldsymbol{t}^{(\boldsymbol{\nu})}
$$

Hence the Cauchy stress lemma introduces the state of stress at each point $\boldsymbol{x} \in \mathcal{B}_{t}$ as

$$
\boldsymbol{t}^{(\boldsymbol{\nu})}(\boldsymbol{x}, t)=\boldsymbol{\sigma}^{T}(\boldsymbol{x}, t) \boldsymbol{\nu}(\boldsymbol{x}, t)
$$

Herein the Cauchy stress tensor $\boldsymbol{\sigma}$ denotes a function of the spatial coordinates of $\boldsymbol{x}$.

If the Cauchy stress principle is referred to a differential surface element in the reference configuration d $A$ with its unit normal vector $\boldsymbol{n}$, the first Piola-Kirchhoff stress tensor $\boldsymbol{P}$ may correspondingly be defined as a function of the material coordinates $\boldsymbol{X}$ by

$$
\boldsymbol{t}^{(\boldsymbol{n})}(\boldsymbol{X}, t)=\boldsymbol{P}(\boldsymbol{X}, t) \boldsymbol{n}(\boldsymbol{X}, t) .
$$

Additionally, the so-called second Piola-Kirchhoff stress tensor $\boldsymbol{S}$ is related to $\boldsymbol{P}$ through

$$
\boldsymbol{P}=\boldsymbol{F S}
$$

Since the resulting differential force $\mathrm{d} \boldsymbol{f}$ acting on a differential surface element $\mathrm{d} a$ in the current configuration and on a differential surface element $\mathrm{d} A$ in the reference configuration are equal, the Cauchy stress $\boldsymbol{\sigma}$ can be directly linked to $\boldsymbol{P}$ by the following relation

$$
\mathrm{d} \boldsymbol{f}=\boldsymbol{P} \boldsymbol{n} \mathrm{d} A=\boldsymbol{\sigma}^{T} \boldsymbol{\nu} \mathrm{d} a=\boldsymbol{\sigma}^{T} \operatorname{det}(\boldsymbol{F}) \boldsymbol{F}^{-T} \boldsymbol{n} \mathrm{d} A
$$


using the definition in Eq. (2.7). Subsequently, $\boldsymbol{P}$ is expressed as

$$
\boldsymbol{P}=\operatorname{det}(\boldsymbol{F}) \boldsymbol{\sigma}^{T} \boldsymbol{F}^{-T}
$$

\subsection{Balance laws}

This section introduces three fundamental balance laws to complete the framework of continuum mechanics needed within this study. These are the balance laws of mass conservation, linear momentum conservation and angular momentum conservation.

\subsubsection{Conservation of mass}

The mass $m$ of a material continuum body $\mathcal{B}_{t}$ at time $t$ in the current configuration is expressed as

$$
m=\int_{\mathcal{B}_{t}} \rho_{t}(\boldsymbol{x}, t) \mathrm{d} v
$$

with $\rho_{t}$ denoting the density at time $t$. In accordance to the law of mass conservation, the total mass of a body $\mathcal{B}$ or of any portion of the body is conserved with motion. Thus, the material derivative of Eq. (2.23) is zero and can be obtained using the relations in Eqs. (2.6) and (2.10) by

$$
\dot{m}=\frac{\mathrm{d}}{\mathrm{d} t} \int_{\mathcal{B}_{t}} \rho_{t}(\boldsymbol{x}, t) \mathrm{d} v=\int_{\mathcal{B}_{t}}\left\{\dot{\rho}_{t}(\boldsymbol{x}, t)+\rho_{t}(\boldsymbol{x}, t) J \operatorname{div} \dot{\boldsymbol{x}}\right\} \mathrm{d} v=0 .
$$

With $\mathcal{B}_{t}$ being any arbitrary part of the continuum, the integrand in Eq. (2.24) must vanish. As a result, the so-called continuity equation in the Eulerian form is expressed as

$$
\dot{\rho}_{t}(\boldsymbol{x}, t)+\rho_{t}(\boldsymbol{x}, t) J \operatorname{div} \dot{\boldsymbol{x}}=0 .
$$

Furthermore, the mass is constant in the current and reference configurations, i.e.

$$
m=\int_{\mathcal{B}_{t}} \rho_{t}(\boldsymbol{x}, t) \mathrm{d} v=\int_{\mathcal{B}} \rho_{0}(\boldsymbol{X}) \mathrm{d} V,
$$


following the conservation of mass and with $\rho_{0}$ denoting the reference mass density. Using the definition of Eq. (2.6) with $\boldsymbol{x}=\boldsymbol{x}(\boldsymbol{X}, t)$, the Lagrangian form of the mass (Eq. 2.23) is given by

$$
m=\int_{\mathcal{B}} \rho_{t}(\boldsymbol{X}, t) J \mathrm{~d} V
$$

Combining Eqs. (2.26) and (2.27) gives

$$
\int_{\mathcal{B}}\left\{\rho_{t}(\boldsymbol{X}, t) J-\rho_{0}(\boldsymbol{X})\right\} \mathrm{d} V=0
$$

This must again be valid for any arbitrary part of the continuum. The continuity equation in the Lagrangian or material form is thus given by

$$
\rho_{t}(\boldsymbol{X}, t) J=\rho_{0}(\boldsymbol{X})
$$

Furthermore, $\dot{\rho}_{0}=0$ is defined by

$$
\frac{\mathrm{d}}{\mathrm{d} t}\left(\rho_{t}(\boldsymbol{X}, t), J\right)=0
$$

This equation enables to evaluate the material derivative of an integral over some product $\rho_{t} A$ through

$$
\frac{\mathrm{d}}{\mathrm{d} t} \int_{\mathcal{B}_{t}} \rho_{t}(\boldsymbol{x}, t) A(\boldsymbol{x}, t) \mathrm{d} v=\int_{\mathcal{B}_{t}} \rho_{t}(\boldsymbol{x}, t) \dot{A}(\boldsymbol{x}, t) \mathrm{d} v
$$

Herein $A(\boldsymbol{x}, t)$ denotes a field of some property per unit mass.

\subsubsection{Linear momentum principle}

Consider a material continuum body $\mathcal{B}$ that is subjected to a body force $\boldsymbol{b}_{t}$ and with its boundary surface $\partial \mathcal{B}$ under the action of a surface traction $\boldsymbol{t}^{(\boldsymbol{\nu})}$. Following the principle of linear momentum, the time rate of change of the linear momentum equals the resultant force acting on the body, i.e.

$$
\frac{\mathrm{d}}{\mathrm{d} t} \int_{\mathcal{B}_{t}} \rho_{t}(\boldsymbol{x}, t) \dot{\boldsymbol{x}} \mathrm{d} v=\int_{\partial \mathcal{B}_{t}} \boldsymbol{t}^{(\boldsymbol{\nu})}(\boldsymbol{x}, t) \mathrm{d} a+\int_{\mathcal{B}_{t}} \boldsymbol{b}_{t}(\boldsymbol{x}, t) \mathrm{d} v
$$


This equation can be modified so that the global equation of motion takes this spatial form

$$
\int_{\mathcal{B}_{t}}\left\{\rho_{t}(\boldsymbol{x}, t) \ddot{\boldsymbol{x}}-\operatorname{div} \boldsymbol{\sigma}^{T}-\boldsymbol{b}_{t}(\boldsymbol{x}, t)\right\} \mathrm{d} v=\mathbf{0}
$$

with the help of the Cauchy stress lemma (Eq. 2.18) and Gauss's divergence theorem. Herein Eq. (2.31) has been used to evaluate the material time derivative. Considering that $\mathcal{B}_{t}$ is arbitrary and therefore the integrand in Eq. (2.32) must vanish, the corresponding local equation of motion then takes the form

$$
\operatorname{div} \boldsymbol{\sigma}^{T}+\boldsymbol{b}_{t}(\boldsymbol{x}, t)=\rho_{t}(\boldsymbol{x}, t) \ddot{\boldsymbol{x}}
$$

If the velocity field $\dot{\boldsymbol{x}}(\boldsymbol{x}, t)$ is constant or zero, the equation of motion reduces to the so-called equilibrium equation as

$$
\operatorname{div} \boldsymbol{\sigma}^{T}+\boldsymbol{b}_{t}(\boldsymbol{x}, t)=\mathbf{0}
$$

Referring all quantities in Eq. (2.33) to the reference configuration yields the global equation of motion in its material form as

$$
\frac{\mathrm{d}}{\mathrm{d} t} \int_{\mathcal{B}} \rho_{0} \dot{\boldsymbol{x}}(\boldsymbol{X}, t) \mathrm{d} V=\int_{\partial \mathcal{B}} \boldsymbol{t}^{(\boldsymbol{n})}(\boldsymbol{X}, t) \mathrm{d} A+\int_{\mathcal{B}} \boldsymbol{b}(\boldsymbol{X}, t) \mathrm{d} V
$$

Replacing the traction vector with the definition in Eq. (2.19), applying the Gauss's divergence theorem and taking the material derivative of the left-hand side give

$$
\int_{\mathcal{B}}\left\{\rho_{0} \ddot{\boldsymbol{x}}(\boldsymbol{X}, t)-\operatorname{Div} \boldsymbol{P}-\boldsymbol{b}(\boldsymbol{X}, t)\right\} \mathrm{d} V=\mathbf{0},
$$

where Div stands for the divergence operator with respect to the reference configuration. The Lagrangian equation of motion can thus be expressed as

$$
\operatorname{Div} \boldsymbol{P}+\boldsymbol{b}(\boldsymbol{X}, t)=\rho_{0} \ddot{\boldsymbol{x}}(\boldsymbol{X}, t) .
$$

If the acceleration field is zero, the Lagrangian equilibrium equation is given by

$$
\operatorname{Div} \boldsymbol{P}(\boldsymbol{X}, t)+\boldsymbol{b}(\boldsymbol{X}, t)=\mathbf{0}
$$




\subsubsection{Angular momentum principle}

The angular momentum is defined as the moment of the linear momentum about a particular reference point, which is usually taken as the origin of a coordinate system. In accordance to the principle of angular momentum, the time rate of change of the moment of linear momentum is equal to the resultant moment of the surface and body forces acting on the body $\mathcal{B}$, i.e.

$$
\frac{\mathrm{d}}{\mathrm{d} t} \int_{\mathcal{B}_{t}} \boldsymbol{x} \times \rho_{t}(\boldsymbol{x}, t) \dot{\boldsymbol{x}} \mathrm{d} v=\int_{\partial \mathcal{B}_{t}} \boldsymbol{x} \times \boldsymbol{t}^{(\boldsymbol{\nu})}(\boldsymbol{x}, t) \mathrm{d} a+\int_{\mathcal{B}_{t}} \boldsymbol{x} \times \boldsymbol{b}_{t}(\boldsymbol{x}, t) \mathrm{d} v
$$

Taking the material derivative of the left-hand side with the help of Eq. (2.37), using the Cauchy stress tensor in Eq. (2.18) and subsequently applying Gauss's divergence theorem gives

$$
\int_{\mathcal{B}_{t}}\left\{\boldsymbol{x} \times \rho_{t}(\boldsymbol{x}, t) \ddot{\boldsymbol{x}}-\operatorname{div}\left(\boldsymbol{x} \times \boldsymbol{\sigma}^{T}\right)-\boldsymbol{x} \times \boldsymbol{b}_{t}(\boldsymbol{x}, t)\right\} \mathrm{d} v=\mathbf{0} .
$$

Herein the divergence term in the current configuration is defined by

$$
\operatorname{div}\left(\boldsymbol{x} \times \boldsymbol{\sigma}^{T}\right)=\operatorname{grad} \boldsymbol{x} \times \boldsymbol{\sigma}^{T}+\boldsymbol{x} \times \operatorname{div} \boldsymbol{\sigma}^{T} .
$$

Simplifying the above equation, the global principle of angular momentum in its spatial form can be expressed by

$$
\int_{\mathcal{B}_{t}}\left\{\boldsymbol{x} \times \rho_{t}(\boldsymbol{x}, t) \ddot{\boldsymbol{x}}+\boldsymbol{\epsilon}: \boldsymbol{\sigma}^{T}-\boldsymbol{x} \times \operatorname{div} \boldsymbol{\sigma}^{T}-\boldsymbol{x} \times \boldsymbol{b}_{t}(\boldsymbol{x}, t)\right\} \mathrm{d} v=\mathbf{0}
$$

Herein $\boldsymbol{\epsilon}$ denotes the basic skew-symmetric third order Levi-Civita tensor (permutation tensor).

The corresponding field equation is thus given by

$$
\boldsymbol{x} \times \rho_{t}(\boldsymbol{x}, t) \ddot{\boldsymbol{x}}-\boldsymbol{x} \times \operatorname{div} \boldsymbol{\sigma}^{T}-\boldsymbol{x} \times \boldsymbol{b}_{t}(\boldsymbol{x}, t)+\boldsymbol{\epsilon}: \boldsymbol{\sigma}^{T}=\mathbf{0},
$$

provided that $\mathcal{B}_{t}$ is arbitrary and the integrand in Eq. (2.43) must thus vanish. Using Eq. (2.34) gives

$$
\boldsymbol{\epsilon}: \boldsymbol{\sigma}^{T}=\mathbf{0},
$$

which demonstrates the symmetric properties of the Cauchy stress tensor. 
The angular momentum principle (Eq. 2.43) in the Lagrangian form can be expressed as

$$
\begin{aligned}
& \frac{\mathrm{d}}{\mathrm{d} t} \int_{\mathcal{B}} \boldsymbol{x}(\boldsymbol{X}, t) \times \rho_{0} \dot{\boldsymbol{x}}(\boldsymbol{X}, t) \mathrm{d} V= \\
& \int_{\partial \mathcal{B}} \boldsymbol{x}(\boldsymbol{X}, t) \times \boldsymbol{t}^{(\boldsymbol{n})}(\boldsymbol{X}, t) \mathrm{d} A+\int_{\mathcal{B}} \boldsymbol{x}(\boldsymbol{X}, t) \times \boldsymbol{b}(\boldsymbol{X}, t) \mathrm{d} V .
\end{aligned}
$$

Taking the material derivative of the left-hand side, replacing the traction vector in the sense of Eq. (2.19) and applying the Gauss's divergence theorem yield

$$
\int_{\mathcal{B}}\left\{\boldsymbol{x}(\boldsymbol{X}, t) \times \rho_{0} \ddot{\boldsymbol{x}}(\boldsymbol{X}, t)-\operatorname{Div}(\boldsymbol{x}(\boldsymbol{X}, t) \times \boldsymbol{P})-\boldsymbol{x}(\boldsymbol{X}, t) \times \boldsymbol{b}(\boldsymbol{X}, t)\right\} \mathrm{d} V=\mathbf{0} .
$$

The evaluation of the divergence term in Eq. (2.47) results in

$$
\int_{\mathcal{B}}\left\{\left(\boldsymbol{x}(\boldsymbol{X}, t) \times\left(\rho_{0} \ddot{\boldsymbol{x}}(\boldsymbol{X}, t)-\operatorname{Div} \boldsymbol{P}-\boldsymbol{b}(\boldsymbol{X}, t)\right)-\operatorname{Grad} \boldsymbol{x}(\boldsymbol{X}, t) \times \boldsymbol{P}\right\} \mathrm{d} V=\mathbf{0} .\right.
$$

Using Eq. (2.38) gives

$$
\int_{\mathcal{B}} \boldsymbol{F} \times \boldsymbol{P} \mathrm{d} V=\mathbf{0}
$$

As the integrand must vanish, i.e.

$$
\boldsymbol{F} \times \boldsymbol{P}=\boldsymbol{\epsilon}:\left(\boldsymbol{F P} \boldsymbol{P}^{T}\right)=\mathbf{0},
$$

the following symmetry condition is obtained

$$
\boldsymbol{F} \boldsymbol{P}^{T}=\boldsymbol{P} \boldsymbol{F}^{T}
$$

The substition of Eq. (2.20) for $\boldsymbol{P}$ demonstrates the symmetry of the second Piola-Kirchhoff tensor, i.e.

$$
\boldsymbol{S}^{T}=\boldsymbol{S}
$$




\subsection{Constitutive formulation of a neo-Hookean material}

This study focuses on the phase field modeling of dynamic britte fracture at finite strains and uses a standard isotropic material model. The formulation under consideration is governed by a compressible neo-Hookean material law associated with a free-energy function. The latter is expressed in terms of the invariants of the right Cauchy-Green tensor $\boldsymbol{C}$, which are defined by

$$
I_{C}=\operatorname{tr} \boldsymbol{C}=\boldsymbol{C}: \mathbf{1} \quad I I_{C}=\frac{1}{2}\left[(\operatorname{tr} \boldsymbol{C})^{2}-\operatorname{tr} \boldsymbol{C}^{2}\right] \quad I I I_{C}=\operatorname{det} \boldsymbol{C}=J^{2} .
$$

Herein "tr" denotes the trace of a tensor, "det" the determinant of a tensor and $J$ the Jacobian (Eq. $2.5)$.

The energy function can be split into volumetric $U(J)$ and deviatoric $\tilde{W}(\boldsymbol{C})$ contributions. This study uses the following definitions for the volumetric function

$$
U(J)=\frac{1}{2} \kappa(J-1)^{2}
$$

and the deviatoric function

$$
\tilde{W}(\boldsymbol{C})=\frac{1}{2} \mu(\operatorname{tr}[\tilde{\boldsymbol{C}}]-3)
$$

Herein the deviatoric component of the right Cauchy-Green tensor is defined by

$$
\tilde{C}=J^{-2 / 3} \boldsymbol{C}
$$

The energy function $W$ can thus be expressed as

$$
\begin{aligned}
W & =\tilde{W}(\boldsymbol{C})+U(J) \\
& =\frac{1}{2} \mu(\operatorname{tr}[\tilde{\boldsymbol{C}}]-3)+\frac{1}{2} \kappa(J-1)^{2} .
\end{aligned}
$$

Herein the shear modulus $\mu>0$ and bulk modulus $\kappa>0$ are related to the Young's modulus $E$ and Poisson's ratio $\nu$ through $\mu=\frac{E}{2(1+\nu)}$ and $\kappa=\frac{E}{3(1-2 \nu)}$.

Using the definition of the energy function (Eq. 2.57), the corresponding second Piola-Kirchoff 
stress tensor $\boldsymbol{S}$ (Eq. 2.52) is derived as

$$
\begin{aligned}
\boldsymbol{S}=2 \frac{\partial W}{\partial \boldsymbol{C}} & =\tilde{\boldsymbol{S}}+\boldsymbol{S}_{\mathrm{vol}} \\
& =\mu J^{-2 / 3}\left(\mathbf{1}-\frac{1}{3} \operatorname{tr}[\boldsymbol{C}] \boldsymbol{C}^{-T}\right)+\kappa(J-1) J \boldsymbol{C}^{-1},
\end{aligned}
$$

with 1 denoting the second-order identity tensor. $\tilde{\boldsymbol{S}}$ and $\boldsymbol{S}_{\mathrm{vol}}$ are respectively the deviatoric and volumetric components of $\boldsymbol{S}$.

Similarly, the fourth-order tangent operator $\mathbb{H}$ can be obtained by

$$
\begin{aligned}
\mathbb{H}=2 \frac{\partial^{2} W}{\partial \boldsymbol{C} \partial \boldsymbol{C}}= & \tilde{\mathbb{H}}+\mathbb{H}_{\mathrm{vol}} \\
= & -\mu J^{-2 / 3}\left(\frac{1}{3}\right)\left[\left(-\left(\frac{1}{3}\right) \operatorname{tr}[\boldsymbol{C}] \boldsymbol{C}^{-1}+\mathbf{1}\right) \otimes \boldsymbol{C}^{-1}+\boldsymbol{C}^{-1} \otimes \mathbf{1}+\operatorname{tr}[\boldsymbol{C}] \boldsymbol{C}^{-1} \otimes \boldsymbol{C}^{-1}\right] \\
& +\kappa\left[\left(2 J^{2} \boldsymbol{C}^{-1}-J \boldsymbol{C}^{-1}\right) \otimes \boldsymbol{C}^{-1}-2(J-1) J \boldsymbol{C}^{-1} \otimes \boldsymbol{C}^{-1}\right] .
\end{aligned}
$$

Herein $\tilde{\mathbb{H}}$ and $\mathbb{H}_{\text {vol }}$ denote respectively the deviatoric and volumetric components of $\mathbb{H}$. 


\section{Chapter 3}

\section{Fundamentals of fracture}

\section{mechanics}

This chapter outlines the basic definitions of fracture mechanics and discusses the Griffith energetic fracture criterion, as comprehensively outlined in Gross and Seelig [40].

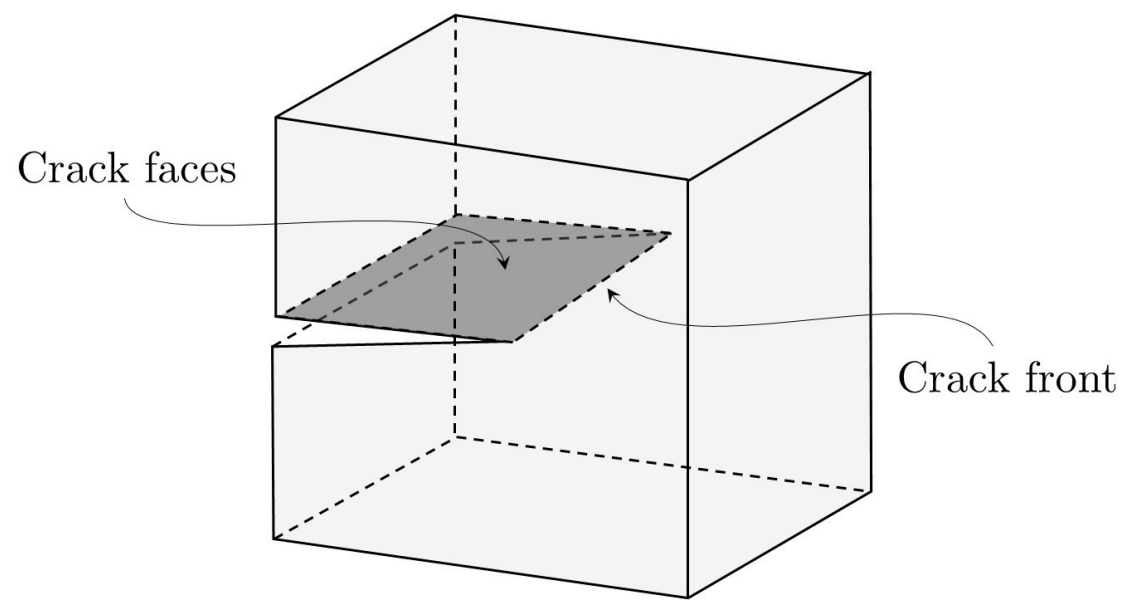

Figure 3.1: Cracked body.

\subsection{Basic definitions}

From a macroscopic perspective, a crack is regarded as a cut in a body. In two dimensions, the crack yields two opposite crack flanks that intersect at the so-called crack tip. In a three-dimensional body, the crack yields two opposite surface boundaries, the so-called crack faces, and ends at the so-called 
crack front, as illustrated in Figure 3.1.

Regarding the deformation of a crack, the opening of a crack can be described with three modes of opening, as depicted in Figure 3.2. The symmetry of the crack opening distinguishes these modes and is defined in the proximity of the crack tip except in cases where the symmetry may hold for the entire body. Mode $I$ is symmetric to the $x-z$ plane. Mode II is antisymmetric with respect to the $x$-direction and is thus normal to the crack front. On the other hand, Mode III is antisymmetric with respect to the $z$-direction. Hence, it is tangential to the crack front.
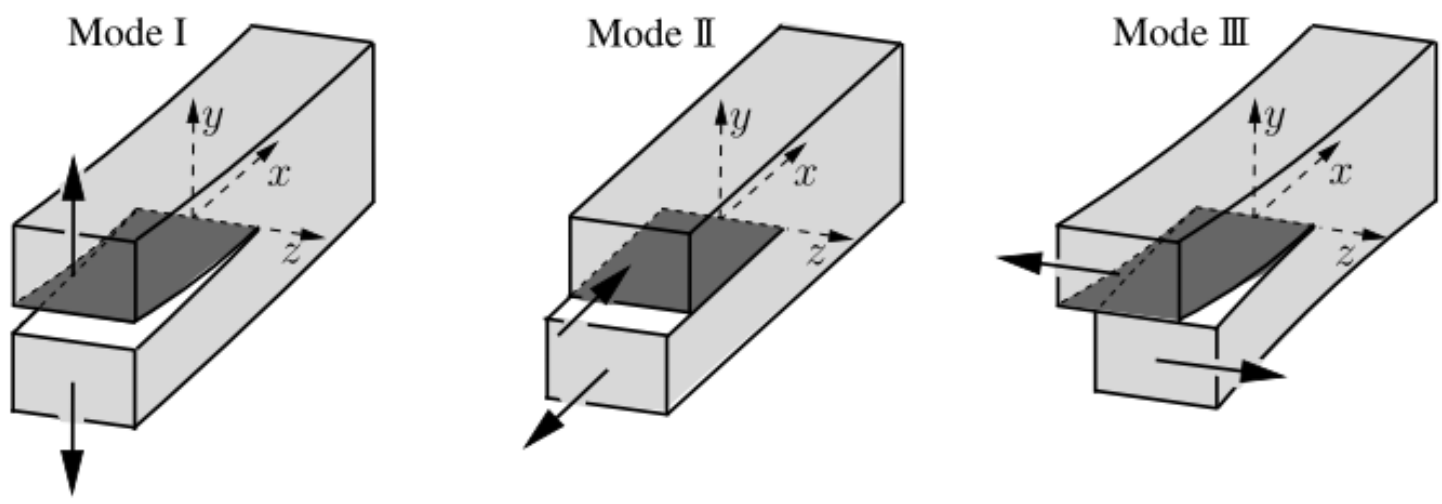

Figure 3.2: Modes of crack opening [61].

Several fracture criteria have been developed to describe the initiation and propagation of cracks. The criteria of Griffith [39] and Irwin [51] are the pioneering works of modern fracture mechanics. Griffith [39] formulated an energetically motivated fracture criterion. In contrast, Irwin [51] proposed the so-called $K$-concept to describe the elastic stress fields near the crack tip using stress intensity factors. Because the phase field model in this study employs the energetic fracture criterion of Griffith, only this criterion is briefly outlined in the following section.

\subsection{Griffith fracture criterion}

Fracture in a body is related to irreversible processes of bond-breaking. All the energies that solely contribute to these processes can be summed up to one term, the so-called fracture surface energy $\Gamma$. It includes the surface energy, and possibly the chemical and electromagnetic energies. The expression of the fracture surface energy takes the following form

$$
\Gamma=\mathcal{G}_{c} A
$$


Herein $\mathcal{G}_{c}$ is a material constant, which is also referred to as the crack resistance or crack resistance force, and $A$ the size of the crack area. Accounting for the fracture surface energy, the energy balance is then expressed as

$$
\dot{E}+\dot{K}+\dot{\Gamma}=\mathcal{W}+\mathcal{D}
$$

where $\dot{E}$ denotes the rate of the store internal energy, $\dot{K}$ the rate of the kinetic energy, $\mathcal{W}$ the rate of work done by external forces and $\mathcal{D}$ the rate of the heat supply.

This balance must hold at the fracture initiation and during the fracture process. Furthermore, the irreversibility of this process must be satisfied for $\dot{\Gamma} \geq 0$. In the case of quasi-static fracture, the fracture process occurs relatively slowly, and the kinetic energy and non-mechanical energy transport (i.e. heat supply) do not play a significant role $(K=0$ and $\mathcal{D}=0)$. Thus, the energy balance in this case is simplified to

$$
\dot{E}+\dot{\Gamma}=\mathcal{W}
$$

For the pure elastic case, the internal energy $E$ can be substituted by the strain energy $\Pi_{\text {int }}$. The external forces can be assumed to possess a potential $\Pi_{\text {ext }}$ and to be conservative. Thus, they have the form $\mathcal{W}=-\frac{\mathrm{d} \Pi_{\text {ext }}}{\mathrm{d} t}$. With the total potential $\Pi=\Pi_{\text {int }}+\Pi_{\text {ext }}$, the energy balance of the quasi-static case (Eq. 3.3) becomes

$$
\frac{\mathrm{d} \Pi}{\mathrm{d} t}+\frac{\mathrm{d} \Gamma}{\mathrm{d} t}=0
$$

For an infinitesimally small crack advance d $A$, this energy balance (Eq. 3.4) can be formally expressed by its equivalent

$$
\left(\frac{\mathrm{d} \Pi}{\mathrm{d} A}+\frac{\mathrm{d} \Gamma}{\mathrm{d} A}\right) \frac{\mathrm{d} A}{\mathrm{~d} t}=0
$$

The release of potential energy upon $\mathrm{d} A$ is the so-called energy release rate $\mathcal{G}$. The latter is expressed by

$$
\mathcal{G}=-\frac{\mathrm{d} \Pi}{\mathrm{d} A}
$$


The crack resistance $\mathcal{G}_{c}$ can be derived from Eq. (3.1) as

$$
\mathcal{G}_{c}=\frac{\mathrm{d} \Gamma}{\mathrm{d} A}
$$

The energy balance (Eq. 3.5) can then be reduced to

$$
\left(\mathcal{G}_{c}-\mathcal{G}\right) \dot{A}=0 .
$$

From Eq. (3.8), the relation between the energy release rate $\mathcal{G}$ and the crack resistance $\mathcal{G}_{c}$ is expressed as

$$
\mathcal{G}=\mathcal{G}_{c}
$$

The equivalent energy relations in Eqs. (3.4) and (3.5) are known as the Griffith fracture criterion. In this condition, the released energy must be equal to the energy required for the initiation of the fracture process and during the subsequent progression of quasi-static fracture advance.

However, Griffith's fracture criterion presents some shortcomings. Griffith assumed that fracture is a reversible process; hence crack healing takes place. Furthermore, he applied this criterion only to initiate the growth of cracks. Thus, it does not predict the path and nucleation of cracks, or the formation of complex topologies due to the kinking and branching of cracks. Additionally, the criterion only considers the case of stable crack propagation, where $\mathcal{G}=\mathcal{G}_{c}$, and the case of continuous crack growth with respect to time. In the case of unstable crack propagation, where $\mathcal{G}$ exceeds $\mathcal{G}_{c}$, the criterion cannot provide any information about the crack evolution.

Given the shortcomings of the Griffith criterion, Francfort and Marigo [33] proposed a variational formulation of quasi-static brittle fracture. A benefit of this formulation is to mainly be consistent with Griffith's theory, departing as little as possible to handle crack nucleation, branching, and interactions between multiple cracks. This improvement nevertheless leads to an increase of computational cost due to the complexity of a direct implementation of sharp discontinuities, particularly in the modeling of complex crack topologies. In addressing the drawback of this formulation, Miehe et al. $[78,79]$ proposed a phase field model for quasi-static fracture at small strains, which was later extended to dynamic fracture by Hofacker and Miehe $[47,48]$ and to finite strains by Miehe and Schänzel [77]. In chapter 4 , the phase field model for dynamic brittle fracture at finite strains is discussed. 


\section{Chapter 4}

\section{Phase field model of dynamic}

\section{brittle fracture}

This chapter introduces a phase field model for dynamic brittle fracture at finite strains. A review on the phase field approximation of a sharp crack topology is first provided following the works of Miehe and Schänzel [77] and Hofacker and Miehe [47, 48]. This approximation is motivated in a one-dimensional setting using a bar with a crack and then generalized to two and three-dimensional settings. A discussion on the degradation of the material bulk energy during the fracture process is outlined. In this regard, the so-called isotropic and anisotropic splits are introduced ${ }^{1}$. The former split does not distinguish the fracture behavior due to tension and compression, while the latter does. Several anisotropic split methods are proposed in the literature, this study however only focuses on the volumetric-deviatoric split presented by Amor et al. [5]. This chapter further discusses the irreversibility of the crack evolution with the introduction of a local history field and briefly outlines alternative models of fracture irreversibility. Lastly, this chapter introduces the variational formulation of the phase field model using the anisotropic split.

\footnotetext{
${ }^{1}$ The terms "isotropic" and "anisotropic" are used in the context of phase field modeling of fracture. They are not related to the conventional notions of material isotropy and anisotropy in continuum mechanics.
} 


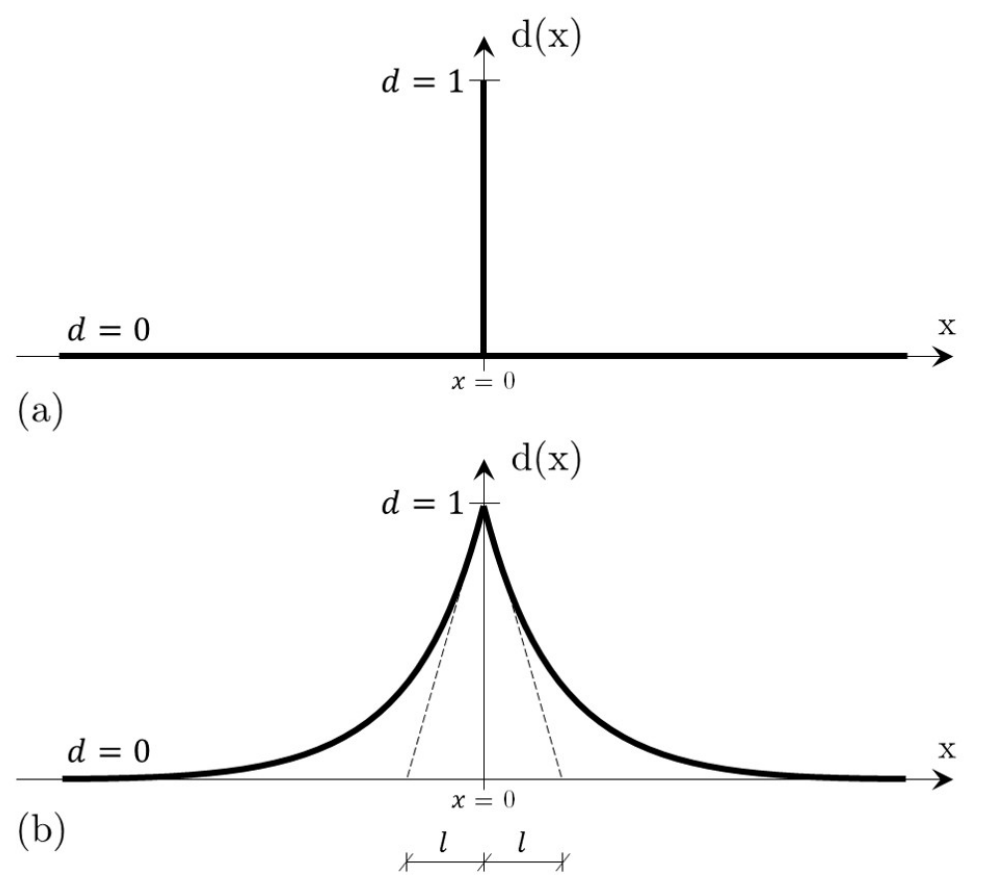

Figure 4.1: Sharp and diffusive crack modeling. (a) Sharp crack at $x=0$ and (b) diffusive crack at $x=0$ modeled with the length-scale $l$.

\subsection{Phase field description of fracture}

\subsubsection{Motivation of the phase field approximation in one dimension}

The phase field method can be motivated using a one-dimensional bar, which has a length $L=$ $[-\infty,+\infty]$ and cross-section $\Gamma$, and occupies the domain $\mathcal{B}=\Gamma \times L$ with the position $x \in L$ defined on the axis. Assuming that the bar contains a crack at the axial position $x=0$ with the crack surface $\Gamma$, this method introduces a phase field variable $d(x) \in[0,1]$ to describe the topology of the sharp crack with

$$
d(x)=\left\{\begin{array}{ll}
1 & \text { for } x=0 \\
0 & \text { otherwise }
\end{array} .\right.
$$

In this description, the phase field varies from $d=0$ in the unbroken state to $d=1$ in the fully broken state, as illustrated in Figure 4.1.

The phase field method resembles that of the damage continuum theory, which also incorporates a scalar field $d$ to capture the growth of micro-cracks and micro-voids in a homogenized macroscopic 
sense. Accordingly, the non-smooth approximation of the phase field yields the exponential function

$$
d(x)=e^{-\frac{|x|}{l}}
$$

with Dirichlet-type boundary conditions

$$
d(0)=1 \quad \text { and } \quad d( \pm \in \infty)=0 .
$$

In essence, the function smears out the crack over the axial domain $L$ of the bar, which results in a regularized or diffusive representation of the crack topology. A length-scale parameter $l$ governs the regularization, controls the width of the smooth approximation of the crack and yields a sharp crack topology $\Gamma$ in the limiting case $l \rightarrow 0$. Furthermore, this parameter controls the critical value of the stress that is required for the nucleation and propagation of cracks.

The exponential function is obtained as the solution of the homogeneous differential equation

$$
d(x)-l^{2} d^{\prime \prime}(x)=0 \quad \text { in } \mathcal{B}
$$

with the Dirichlet-type boundary conditions in Eq. (4.3). This equation constitutes the Euler equation of the variational principle

$$
d=\operatorname{Arg}\left\{\inf _{d \in W} I(d)\right\}
$$

subjected to Dirichlet boundary conditions $W=\{d \mid d(0)=1, d( \pm \in \infty)=0\}$ and as a function of

$$
I(d)=\frac{1}{2} \int_{\mathcal{B}}\left\{d^{2}+l^{2} d^{\prime 2}\right\} \mathrm{d} V .
$$

The functional $I(d)$ can be obtained with the integration of a Galerkin-type weak form of the differential equation (Eq. 4.4). Inserting the exponential function (Eq. 4.2) into Eq. (4.6) and evaluating $\mathrm{d} V=\Gamma \mathrm{d} x$ yields the following relationship between the crack surface $\Gamma$ and this functional

$$
I\left(d=e^{-|x| / l}\right)=l \Gamma .
$$


Dividing the functional (Eq. 4.6) by the length-scale parameter 1 gives

$$
\Gamma_{l}(d)=\frac{1}{l} I(d)=\frac{1}{2 l} \int_{\mathcal{B}}\left\{d^{2}+l^{2} d^{\prime 2}\right\} \mathrm{d} V .
$$

The minimization of the scaled functional $\Gamma_{l}(d)$ (Eq. 4.8) yields the regularized crack topology (Eq. 4.4) depicted in Figure 4.1(b). This scaled functional may be regarded as the crack surface itself. Correspondingly, the evaluation of $\Gamma_{l}(d)$ at the solution point $x=0$ gives the crack surface $\Gamma$ for any arbitrary length-scale $l$. Hence, the functional $\Gamma_{l}$ is an important property in the constitutive modeling of crack propagation.

\subsubsection{Motivation of the phase field approximation in two and three di- mensions}

The regularization of the sharp crack topology in one dimension, discussed above, can be generalized to two- and three- dimensional settings. Assume $\mathcal{B} \subset \mathcal{R}^{\delta}$ is a material body in the reference configuration with surface $\partial \mathcal{B} \subset \mathcal{R}^{\delta-1}$ and dimension $\delta \in[2,3]$ in space. The phase field method introduces a time-dependent scalar phase field variable $d(\boldsymbol{X}, t) \in[0,1]$ to describe the evolution of cracks inside the body, where $\boldsymbol{X}$ denotes the particle in the Lagrangian configuration and $t$ the time. See Figure 4.2 for the two-dimensional illustration of the body with internal discontinuities using sharp and diffusive representations of the crack.

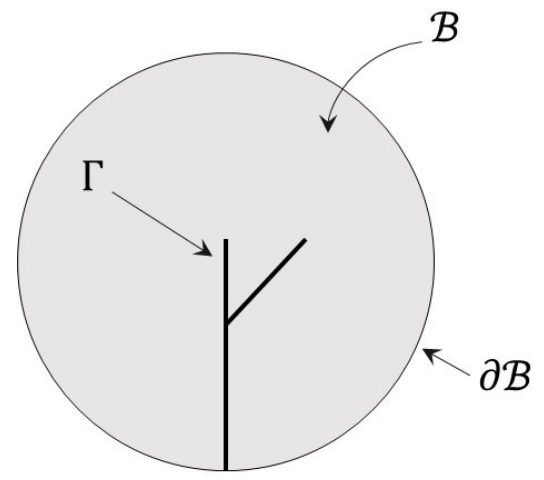

(a) Sharp crack

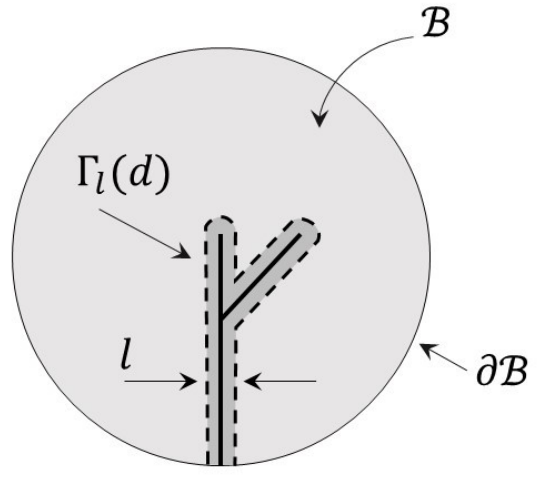

(b) Diffusive crack

Figure 4.2: (a) Body with internal discontinuities (sharp cracks) $\Gamma$ and (b) Approximation of internal discontinuities using the phase field $d(\boldsymbol{X}, t)$. 
The regularized functional (Eq. 4.8) can be expressed in the two- and three- dimensional settings as

$$
\Gamma_{l}(d)=\int_{\mathcal{B}} \gamma_{l}(d, \nabla d) \mathrm{d} V
$$

Herein $\gamma_{l}$ denotes the $n$ th-order crack surface density function per unit volume of the solid and is governed by the length-scale parameter $l$. Its commonly used expression is given by the second-order Allen-Cahn energy density function

$$
\gamma_{l}(d, \nabla d)=\frac{1}{2 l} d^{2}+\frac{l}{2}|\nabla d|^{2}
$$

in terms of the crack phase field $d$ and its spatial gradient $\nabla d$. This function is essential to model the propagation of cracks. The crack topology can also be regularized with a higher-order function for better accuracy and convergence rate of numerical solutions. For further insight into higher-order regularization functions, the reader is referred to the works of Borden [11] and Weinberg and Hesch [117].

Wth Eq. (4.9), the regularized fracture surface energy $W_{\text {frac }}$, which is a volume integral approximating the surface integrals defined on sharp crack surfaces $\Gamma \subset \mathcal{B}$, is expressed as

$$
W_{\text {frac }}(d, \nabla d)=\int_{\Gamma} \mathcal{G}_{c} \mathrm{~d} \Gamma \approx \int_{\Gamma} \mathcal{G}_{c} \gamma_{l}(d, \nabla d) \mathrm{d} V \approx \int_{\Gamma} \mathcal{G}_{c}\left[\frac{1}{2 l} d^{2}+\frac{l}{2}|\nabla d|^{2}\right] \mathrm{d} V
$$

Herein $\mathcal{G}_{c}$ denotes the Griffith critical energy release.

\subsection{Degradation of the body's bulk energy}

\subsubsection{Isotropic split}

The simplest definition of the damaged bulk energy $W_{\text {bulk }}$ consists of multiplying the undamaged bulk energy $W$ (Eq. 2.57) by a degradation function $g(d)$ as

$$
W_{\text {bulk }}(\boldsymbol{C}, d)=g(d) W(\boldsymbol{C})
$$

This approach, commonly known as the isotropic split, allows cracks to develop in cases of tension, shear and compression, and does not prevent the interpenetration of crack faces under compression [2]. $g(d)$ is a monotonically decreasing degradation function, which models the impact of the phase 
field on the bulk energy. The properties of this function are

$$
g(0)=1, \quad g(1)=0 \quad \text { and } \quad g^{\prime}(1)=0
$$

The first two properties specify the limits for the unbroken and fully broken states. The last property guarantees the convergence of the elastic fracture force to a final value as the material is being damaged up to the fully broken state $d=1$. The degradation function commonly takes the form of a quadratic function, given by

$$
g(d)=(1-d)^{2}
$$

However, cubic and quartic functions are also available in literature, as outlined in Kuhn et al. [64] and Borden [11]. An enhanced type of the degradation function is also introduced by Steinke and Kaliske [110]. The authors found that this novel type leads to a more brittle behavior of the phase field model. These alternative degration functions are not considered in this thesis, but can be considered in future studies.

The damaged bulk energy in Eq. (4.12) does not ensure that the algebraic conditioning number of the numerical discretization method is well-posed for partly or fully broken systems. Thus, numerical singularities can occur in these systems. To address this shortcoming, a small positive parameter $k \approx 0$ is introduced. This parameter is chosen as small as possible and induces a artificial elastic rest energy density $k W$ when the energy is fully degraded at a fully broken state $d=1$.

With the introduction of $k$, Eq. (4.12) becomes

$$
W_{b u l k}(\boldsymbol{C}, d)=[g(d)+k] W(\boldsymbol{C})
$$

An important feature of the phase field model is the energetic force $f$ which is the driving term of the crack evolution. It is expressed as

$$
f=-\frac{\partial W}{\partial d}=2(1-d) W(\boldsymbol{C})
$$

and bounded by a finite value for the limiting case of $W \rightarrow \infty$. This feature guarantees that the maximum value of the phase field $d$ is restricted to 1.

Another feature is the micro force $\kappa$, which is the thermodynamical conjugate to the rate of the 
phase field $\dot{d}[61]$, given by

$$
\kappa=\frac{\partial W_{f r a c}}{\partial \nabla d}=\mathcal{G}_{c} l \nabla d
$$

\subsubsection{Anisotropic split}

In contrast to the isotropic split (Eq. 4.15), the anisotropic split captures a more realistic fracture pattern by enforcing that the fracture propagation only occurs due to tension and shear and by prohibiting the propagation under compression. This split is particularly important for dynamic simulations. In the latter case, compressive stress waves that reflect from domain boundaries have a tendancy to create fracture patterns which are physically unrealistic when the compressive energy is applied to the phase field [13]. Consequently, the anisotropic split additively decomposes the undamaged bulk energy $W$ to distinguish between the tensile part $W^{+}$and the compressive part $W^{-}$as follows

$$
W=W^{+}+W^{-} .
$$

With this decomposition, the damaged bulk energy (Eq. 4.15) reads

$$
W=[g(d)+k] W^{+}+W^{-}
$$

As a result, the degradation function only affects the tensile part of the energy density. In case the strains enter a fully compressive range, the anisotropic split accounts for crack closure and prevents the interpretation of the crack faces.

With regards to the definition of the tensile and compressive parts of the bulk energy, several approaches have been proposed in literature. This study adopts the volumetric-deviatoric split of Amor et al. [5]. This split allows the evolution of crack to occur only due to volumetric expansion with the Jacobian $J>1$ (Eq. 2.5) and deviatoric deformation but not volumetric compression where $J<1$. Hence, this approach involves the decomposition of the bulk energy into volumetric and 
deviatoric contributions. In this regard, the tensile and compressive parts are expressed as

$$
\begin{aligned}
& W^{+}= \begin{cases}\tilde{W}(\boldsymbol{C})+U(J) & J \geqslant 1 \\
\tilde{W}(\boldsymbol{C}) & J<1\end{cases} \\
& W^{-}= \begin{cases}0 & J \geqslant 1 \\
U(J) & J<1\end{cases}
\end{aligned}
$$

in terms of the invariants of the right Cauchy-Green tensor $\boldsymbol{C}$. Herein $U(J)($ Eq. 2.54) and $\tilde{W}(\boldsymbol{C})$ (Eq. 2.55) denote the deviatoric and volumetric parts of the bulk energy, respectively.

Thus, Eq. (4.19) becomes

$$
W_{\text {bulk }}(\boldsymbol{C}, d)=[g(d)+k] W^{+}(\boldsymbol{C}, J)+W^{-}(J)
$$

For further insight into alternative splits, the reader is referred to the spectral decomposition of the strain tensor at small strains presented by Miehe et al. [78, 79]. This split assumes that cracks evolve due to volumetric expansion and without reference to the case of a pure deviatoric strain state. As a result, the formulation involves a spectral decomposition of bulk energy into the principal strains and their corresponding principal directions. Hesch et al. [45] adopted a similar split to Miehe et al. $[78,79]$ but used the decomposition in terms of the principal invariants. In contrast, Steinke and Kaliske [110] introduced a directional split which is based on the decomposition of the stress tensor with respect to the crack orientation into crack driving and persistent components. In this split, they also specified a local crack coordinate system. Accordingly, they presented a modified stress-strain relation for the modeling of fundamental crack characteristics.

Following the anisotropic split, the energetic force $f$ driving the crack is thus defined by

$$
f=-\frac{\partial W}{\partial d}=2(1-d) W^{+}(\boldsymbol{C}, J)
$$

which is consequently always positive. Furthermore, it is bounded by a finite value for the limiting case of $W^{+} \rightarrow \infty$. Like in the isotropic split, this feature guarantees that the maximum value of the phase field $d$ is restricted to 1 . Contrary to the isotropic split, only the positive part $W^{+}$of the reference energy is the driving term of the crack evolution. This part is related to the local intensity of the tensile and shear parts of the deformation and thus ensures that only tension and shear forces 
can cause the propagation of fracture.

As a result of the degradation of the energy (Eq. 4.22) and taking into account the phase field $d$, the second Piola-Kirchoff stress tensor (Eq. 2.58) is derived from

$$
\boldsymbol{S}=[g(d)+k] \boldsymbol{S}^{+}+\boldsymbol{S}^{-}
$$

Herein $\boldsymbol{S}^{+}$and $\boldsymbol{S}^{-}$denote respectively the positive and negative stress tensors. The latter are defined, with the help of the neo-Hookean energy function expressed in Eq. (2.57) and the expressions of the tensile and compressive parts of the bulk energy respectively in Eqs. (4.20) and (4.21), as

$$
\begin{aligned}
& \boldsymbol{S}^{+}=2 \frac{\partial W^{+}}{\partial \boldsymbol{C}}= \begin{cases}\mu J^{-2 / 3}\left(\mathbf{1}-\frac{1}{3} \operatorname{tr}[\boldsymbol{C}] \boldsymbol{C}^{-T}\right)+\kappa(J-1) J \boldsymbol{C}^{-1} & J \geqslant 1 \\
\mu J^{-2 / 3}\left(\mathbf{1}-\frac{1}{3} \operatorname{tr}[\boldsymbol{C}] \boldsymbol{C}^{-T}\right) & J<1\end{cases} \\
& \boldsymbol{S}^{-}=2 \frac{\partial W^{-}}{\partial \boldsymbol{C}}=\left\{\begin{array}{ll}
0 & J \geqslant 1 \\
\kappa(J-1) J \boldsymbol{C}^{-1} & J<1
\end{array} .\right.
\end{aligned}
$$

With the definitions of the quadratic degradation function in Eq. (4.14), the positive and negative tensors in Eqs. (4.25) and (4.26), the second Piola-Kirchoff stress tensor (4.24) now reads

$$
\boldsymbol{S}=\left\{\begin{array}{l}
{\left[(1-d)^{2}+k\right]\left[\mu J^{-2 / 3}\left(\mathbf{1}-\frac{1}{3} \operatorname{tr}[\boldsymbol{C}] \boldsymbol{C}^{-T}\right)+\kappa(J-1) J \boldsymbol{C}^{-1}\right] \quad J \geqslant 1} \\
{\left[(1-d)^{2}+k\right]\left[\mu J^{-2 / 3}\left(\mathbf{1}-\frac{1}{3} \operatorname{tr}[\boldsymbol{C}] \boldsymbol{C}^{-T}\right)\right]+\kappa(J-1) J \boldsymbol{C}^{-1} \quad J<1}
\end{array} .\right.
$$

Correspondingly, the fourth-order tangent operator $\mathbb{H}$ (Eq. 2.59) is obtained from

$$
\mathbb{H}=[g(d)+k] \mathbb{H}^{+}+\mathbb{H}^{-}
$$


Herein the positive and negative fourth-order tangent operators are expressed as

$$
\begin{aligned}
\mathbb{H}^{+} & =2 \frac{\partial^{2} W^{+}}{\partial \boldsymbol{C} \partial \boldsymbol{C}} \\
& = \begin{cases}-\mu J^{-2 / 3}\left(\frac{1}{3}\right) \\
{\left[\left(-\left(\frac{1}{3}\right) \operatorname{tr}[\boldsymbol{C}] \boldsymbol{C}^{-1}+\mathbf{1}\right) \otimes \boldsymbol{C}^{-1}+\boldsymbol{C}^{-1} \otimes \mathbf{1}+\operatorname{tr}[\boldsymbol{C}] \boldsymbol{C}^{-1} \otimes \boldsymbol{C}^{-1}\right]} \\
+\kappa\left[\left(2 J^{2} \boldsymbol{C}^{-1}-J \boldsymbol{C}^{-1}\right) \otimes \boldsymbol{C}^{-1}-2(J-1) J \boldsymbol{C}^{-1} \otimes \boldsymbol{C}^{-1}\right] & J \geqslant 1 \\
-\mu J^{-2 / 3}\left(\frac{1}{3}\right)\left[\left(-\left(\frac{1}{3}\right) \operatorname{tr}[\boldsymbol{C}] \boldsymbol{C}^{-1}+\mathbf{1}\right) \otimes \boldsymbol{C}^{-1}+\boldsymbol{C}^{-1} \otimes \mathbf{1}+\operatorname{tr}[\boldsymbol{C}] \boldsymbol{C}^{-1} \otimes \boldsymbol{C}^{-1}\right] & J<1\end{cases}
\end{aligned}
$$

and

$$
\mathbb{H}^{-}=2 \frac{\partial^{2} W^{-}}{\partial \boldsymbol{C} \partial \boldsymbol{C}}=\left\{\begin{array}{ll}
0 & J \geqslant 1 \\
\kappa\left[\left(2 J^{2} \boldsymbol{C}^{-1}-J \boldsymbol{C}^{-1}\right) \otimes \boldsymbol{C}^{-1}-2(J-1) J \boldsymbol{C}^{-1} \otimes \boldsymbol{C}^{-1}\right] & J<1
\end{array},\right.
$$

respectively.

The additional tangent operators are $\mathbb{H}_{d \boldsymbol{u}}$ and $\mathbb{H}_{\boldsymbol{u} d}$. The former is defined using the definitions of the tensile body's bulk energy $W^{+}$(Eq. 4.20), energetic force $f$ (Eq. 4.23) and positive stress tensor $\boldsymbol{S}^{+}$(Eq. 4.25) as

$$
\begin{aligned}
\mathbb{H}_{d \boldsymbol{u}} & =2 \frac{\partial f}{\partial \boldsymbol{C}}=2 \frac{\partial\left[(1-d) W^{+}\right]}{\partial \boldsymbol{C}}=2(1-d) \boldsymbol{S}^{+}, \\
& =2(1-d) \begin{cases}\mu J^{-2 / 3}\left(\mathbf{1}-\frac{1}{3} \operatorname{tr}[\boldsymbol{C}] \boldsymbol{C}^{-T}\right)+\kappa(J-1) J \boldsymbol{C}^{-1} & J \geqslant 1 \\
\mu J^{-2 / 3}\left(\mathbf{1}-\frac{1}{3} \operatorname{tr}[\boldsymbol{C}] \boldsymbol{C}^{-T}\right) & J<1\end{cases}
\end{aligned}
$$

The latter is expressed with the help of the degradation function $g(d)$ (Eq. 4.14), the second PiolaKirchoff tensor $\boldsymbol{S}$ (Eq. 4.24) and the positive stress tensor $\boldsymbol{S}^{+}$(Eq. 4.25) as

$$
\begin{aligned}
\mathbb{H}_{\boldsymbol{u} d} & =\frac{\partial \boldsymbol{S}}{\partial d}=\frac{\partial\left\{[g(d)+k] \boldsymbol{S}^{+}+\boldsymbol{S}^{-}\right\}}{\partial d}=\frac{\partial\left\{\left[(1-d)^{2}+k\right] \boldsymbol{S}^{+}+\boldsymbol{S}^{-}\right\}}{\partial d}=-2(1-d) \boldsymbol{S}^{+} \\
& =-2(1-d)\left\{\begin{array}{lc}
\mu J^{-2 / 3}\left(\mathbf{1}-\frac{1}{3} \operatorname{tr}[\boldsymbol{C}] \boldsymbol{C}^{-T}\right)+\kappa(J-1) J \boldsymbol{C}^{-1} & J \geqslant 1 \\
\mu J^{-2 / 3}\left(\mathbf{1}-\frac{1}{3} \operatorname{tr}[\boldsymbol{C}] \boldsymbol{C}^{-T}\right) & J<1
\end{array} .\right.
\end{aligned}
$$




\subsection{Irreversibility of the fracture process}

The irreversibility of the fracture process means that all cracks must only extend over time, i.e. $\Gamma(t) \subseteq \Gamma(t+\Delta t)$ for all $\Delta t>0$. Accordingly, the phase field model must enforce this irreversibility to be physically consistent. Several irreversibility approaches have been developed for this enforcement. This study employs the approach of Miehe et al. [78, 79] which introduces a rate-independent local history field of the maximum positive reference energy through the use of a local damage model in one dimension. This approach is motivated by the observation that a "load" term governs the amount of the phase field variable $d$ in the loading case. This term is $W_{\text {bulk }}(4.16)$ in the isotropic split and $W^{+}(4.23)$ in the anisotropic split. The maximum energy is determined in a typical and possibly cyclical loading process.

With the phase field $d$ and local history field $\mathcal{H}$, the following physically based restrictions can be considered

$$
d \in[0,1], \quad \dot{d} \geq 0 \quad \text { and } \quad \mathcal{H} \geq 0
$$

which ensures that the phase field grows up to the fully broken state. Eq. $(4.33)_{(1)}$ is a local condition which provides the boundary between the initial condition $d=0$ with no cracks and the final condition $d=1$ when cracks accumulate. The one-dimensional relationship between $d$ and $\mathcal{H}$ is given by

$$
d=\frac{\mathcal{H}}{1+\mathcal{H}}
$$

Applying the restrictions in Eq. (4.33) to this relationship gives

$$
d=\frac{\mathcal{H}}{1+\mathcal{H}} \in[0,1] \quad \text { and } \quad \dot{d}=\frac{\dot{\mathcal{H}}}{(1+\mathcal{H})^{2}} \geq 0
$$

where $\dot{\mathcal{H}}$ denotes the evolution of the local history field .

From Eq. $\left(4.35_{(1)}\right)$, the relations between the boundary states and the local history field are defined by

$$
d=0 \Leftrightarrow \mathcal{H}=0 \quad \text { and } \quad d \rightarrow 1 \Leftrightarrow \mathcal{H} \rightarrow \infty .
$$


With these relations and the irreversibility restriction of the phase field (Eq. $\left.4.33_{(2)}\right)$, the history field $\mathcal{H}$ must satisfy the constraint

$$
\frac{d}{d t} \mathcal{H} \geq 0
$$

As a result, the field $\mathcal{H}$ is a positive and monotonously growing function and is expressed as

$$
\mathcal{H}(\boldsymbol{X}, t)=\max _{s \in[0, t]} W_{b u l k}(\boldsymbol{C}(\boldsymbol{X}, s)) \quad \text { and } \quad \mathcal{H}(\boldsymbol{X}, t)=\max _{s \in[0, t]} W^{+}(\boldsymbol{C}(\boldsymbol{X}, s))
$$

in the isotropic and anisotropic splits, respectively. This field models the irreversibility condition of the fracture process by ensuring that the maximum bulk energy in the material during the full process history determines the present stiffness, and thus ensures that no crack healing takes place. The bulk energy $W_{\text {bulk }}$ is positive, independent of the phase field $d$ and drives its accumulation.

In the case of anisotropic split, replacing $W^{+}$in Eq. (4.23) by the local history field gives

$$
f=-\frac{\partial W}{\partial d}=2(1-d) \mathcal{H}
$$

Thus, the tangent operator $\mathbb{H}_{d \boldsymbol{u}}$ (Eq. 4.31) can be expressed as

$$
\mathbb{H}_{d \boldsymbol{u}}=2 \frac{\partial[2(1-d) \mathcal{H}]}{\partial \boldsymbol{C}}
$$

This irreversibility approach is computationally efficient because the only computational cost is the requirement to store one history variable per integration point. Additionally, initial cracks can be modeled using the initial history field. However, the thermodynamic justification may not hold for a non-local damage model. Despite this possible shortcoming, this approach may still be a good choice for many practical cases [11].

Several alternative approaches can also be used to model the irreversibility of the fracture process. One approach consists of enforcing the monotonicity of the phase field $d$ by satisfying the condition $\dot{d} \geq 0$ for all time $t$. This approach constrains the minimization problem with the associated additional computational cost. Another approach proposed by Schlüter et al. [101] consists of defining Dirichlet boundary conditions for the phase field such that when the phase field reaches the broken state at a point in time, it remains broken for the subsequent time. Furthermore, the phase field can recover to the unbroken state if it has not reached the broken state. As a result, the full stiffness of 
the material can be retained even if the material is loaded close to failure in a previous loading cycle. This characteristic is typical of brittle fracture. Bourdin et al. [15] proposed a different approach which constrains a subset of the discrete phase field control variables that meet a minimum value threshold. These alternative approaches are not considered in this study but may be considered in future works.

\subsection{Variational formulation for dynamic fracture}

To address dynamics problems, a kinetic energy, which is not dependent on the phase field $d$, is introduced. Let the system under consideration be Hamiltonian. The Hamiltonian as the total energy is then defined in terms of the kinetic energy $\mathcal{K}$, the internal power $\Psi_{\text {int }}$ and the external power $\Psi_{e x t}$ as

$$
H=\mathcal{K}+\Psi_{i n t}+\Psi_{\text {ext }}
$$

in the reference configuration [100]. Herein $\mathcal{K}$ is expressed as

$$
\mathcal{K}=\frac{1}{2} \int_{\mathcal{B}} \rho_{0} \dot{\boldsymbol{x}} \cdot \dot{\boldsymbol{x}} \mathrm{d} V
$$

In the presence of damage and using the anisotropic split, $\Psi_{i n t}$ is given as a function of the damaged bulk energy (Eq. 4.19) and crack surface energy (Eq. 4.11). It takes the form

$$
\left.\Psi_{\text {int }}(\boldsymbol{C}, d, \boldsymbol{\nabla} d)=\int_{\mathcal{B}}[g(d)+k] W^{+}(\boldsymbol{C})+W^{-}\right] \mathrm{d} V+\int_{\mathcal{B}} W_{f r a c}(d, \boldsymbol{\nabla} d) \mathrm{d} V .
$$

$\Psi_{\text {ext }}$ is expressed as

$$
\Psi_{e x t}=-\int_{\mathcal{B}} \boldsymbol{b} \cdot \boldsymbol{u} \mathrm{d} V-\int_{\partial \mathcal{B}_{N}} \boldsymbol{t}^{(\boldsymbol{\nu})} \cdot \boldsymbol{u} \mathrm{d} A
$$

where $\boldsymbol{b}$ denotes the body force and $\boldsymbol{t}^{(\boldsymbol{\nu})}$ the external traction vector prescribed on Neumann boundary conditions $\partial \mathcal{B}_{N}$. The Hamilton's principle states that the Hamiltonian and the corresponding Lagrangian,

$$
\mathcal{L}=\mathcal{K}-\Psi
$$


are constant between two points in time $t_{0}$ and $t_{1}$ in the absence of dissipation, with $\Psi=\Psi_{i n t}+\Psi_{\text {ext }}$. Consequently, the variation of the Lagrangian vanishes,

$$
\delta \int_{t_{0}}^{t_{1}}\left\{\frac{1}{2} \int_{\mathcal{B}} \rho_{0} \dot{\boldsymbol{x}} \cdot \dot{\boldsymbol{x}} \mathrm{d} V-\int_{\mathcal{B}} \Psi_{i n t} \mathrm{~d} V+\int_{\mathcal{B}} \boldsymbol{b} \cdot \boldsymbol{u} \mathrm{d} V+\int_{\partial \mathcal{B}_{N}} \boldsymbol{t}^{(\boldsymbol{\nu})} \cdot \boldsymbol{u} \mathrm{d} A\right\} \mathrm{d} t=0 .
$$

Using the relation in Eq. (2.13) between the right Cauchy-Green strain tensor $\boldsymbol{C}$ and the Green strain tensor $\boldsymbol{E}$, the variation of the internal power (Eq. 4.43), can be expressed as

$$
\begin{aligned}
\delta \Psi_{i n t} & =\int_{\mathcal{B}}\left\{\frac{\partial \Psi}{\partial \boldsymbol{E}}: \delta \boldsymbol{E}+\frac{\partial \Psi}{\partial d} \delta d+\frac{\partial \Psi}{\partial \boldsymbol{\nabla} d} \cdot \delta \boldsymbol{\nabla} d\right\} \mathrm{d} V \\
& =\int_{\mathcal{B}}\left\{\boldsymbol{S}: \delta \boldsymbol{E}-f \delta d+\frac{\partial W_{\text {frac }}}{\partial d} \delta d+\boldsymbol{\kappa} \cdot \delta \boldsymbol{\nabla} d\right\} \mathrm{d} V,
\end{aligned}
$$

where $\boldsymbol{S}$ denotes the second Piola-Kirchhoff stress tensor (Eq. 4.27), $f$ the energetic force (Eq. $4.23)$ and $\boldsymbol{\kappa}$ the micro force (Eq. 4.17), $\delta \boldsymbol{E}$ the variation of the Green strain tensor, $\delta d$ and $\delta \boldsymbol{\nabla} d$ the respective variations of the phase field and its gradient.

Applying the Gauss's divergence theorem with respect to time and considering the fact that the variations $\delta \boldsymbol{u}$ and $\delta d$ at the time boundaries $t_{0}$ and $t_{1}$ vanish, the following is obtained

$$
\begin{aligned}
& \int_{\mathcal{B}} \rho_{0} \ddot{\boldsymbol{x}} \cdot \delta \boldsymbol{u} \mathrm{d} V-\int_{\mathcal{B}}\left\{\boldsymbol{S}: \delta \boldsymbol{E}-f \delta d+\frac{\partial W_{\text {frac }}}{\partial d} \delta d+\boldsymbol{\kappa} \cdot \delta \boldsymbol{\nabla} d\right\} \mathrm{d} V \\
& -\int_{\mathcal{B}} \boldsymbol{b} \cdot \delta \boldsymbol{u} \mathrm{d} V-\int_{\partial \mathcal{B}_{N}} \boldsymbol{t}^{(\boldsymbol{\nu})} \cdot \delta \boldsymbol{u} \mathrm{d} A=0 .
\end{aligned}
$$

The governing equations of the above functional are the equation of motion in Eq. (2.38) and the evolution equation of the phase field, which is given by

$$
2(1-d) \mathcal{H}+\frac{\mathcal{G}_{c}}{l} d+\mathcal{G}_{c} l \Delta d=0
$$

Herein $\Delta$ denotes the Laplace operator. This functional is supplemented by Dirichlet boundary conditions and Neumann boundary conditions for the displacement. Furthermore, the boundary conditions for the phase field are defined by $\boldsymbol{\nabla} d \cdot \boldsymbol{n}=0$ on $\partial \mathcal{B}$. 


\section{Chapter 5}

\section{Finite element implementation}

This chapter outlines the implementation of the dynamic phase field model introduced in Chapter 4. The in-house software SESKA ${ }^{1}$, which can support a standard finite element approximation, is used for the implementation. This chapter first provides the weak forms of the motion equation and the evolution equation of the phase field. Then, the global coupled system of equations is defined. Due to the nonlinearity of this system, this study takes advantage of the already implemented nonlinear solver using the Newton-Raphson algorithm with an automatic time-stepping scheme within the SESKA software. Algorithmically, the implementation of the phase field model can be carried out using a monolithic or staggered scheme. In this regard, this chapter briefly discusses both schemes. However, the implementation only considers the monolithic scheme. This chapter further discusses the finite element interpolation procedures. At the end of the chapter, the approximations of the residual vector, tangent matrix, damping matrix, and mass matrix are also explicitly provided. For further insight into the finite element method, several comprehensive books are available in literature; see e.g., Zienkiewicz [122], Hughes [50] and Wriggers [118].

\footnotetext{
${ }^{1}$ More details on the in-house software SESKA are provided in Appendix A.
} 


\subsection{Weak forms and spatial discretization}

From the variation in Eq. (4.48) and assuming no volume forces (i.e. $\boldsymbol{b}=\mathbf{0}$ ), the weak forms of motion equation and the evolution equation of the phase field can be respectively formulated as

$$
\begin{aligned}
& \int_{\mathcal{B}}\left\{\boldsymbol{S}: \delta \boldsymbol{E}+\rho_{0} \ddot{\boldsymbol{x}} \cdot \delta \boldsymbol{u}\right\} \mathrm{d} V-\int_{\partial \mathcal{B}_{N}} \boldsymbol{t}^{(\boldsymbol{\nu})} \cdot \delta \boldsymbol{u} \mathrm{d} A=0 \\
& \int_{\mathcal{B}}\left\{2(1-d) \mathcal{H} \delta d+\frac{\mathcal{G}_{c}}{l} d \delta d+\mathcal{G}_{c} l \nabla d \cdot \delta \boldsymbol{\nabla} d\right\} \mathrm{d} V=0 .
\end{aligned}
$$

The discretization of the initial configuration $\mathcal{B}$ into $n_{e}$ non-overlapping elements, such that $\mathcal{B} \approx$ $\bigcup_{e=1}^{n_{e}} \mathcal{B}^{(e)}$, is achieved through standard isoparametric finite elements. As a result, the displacement field $\boldsymbol{u}$ and phase field $d$, their variations $\delta \boldsymbol{u}$ and $\delta d$, as well as their increments $\Delta \boldsymbol{u}$ and $\Delta d$, are interpolated within an element domain using quadratic shape functions $N_{I}^{\boldsymbol{u}}$ and $N_{I}^{d}$ in this work.

The numerical approximations of the displacement field $\boldsymbol{u}$, its variation $\delta \boldsymbol{u}$, its increment $\Delta \boldsymbol{u}$ as well as their spatial derivatives can be then defined by

$$
\boldsymbol{u}_{h}=\sum_{I=1}^{N_{e}} N_{I}^{\boldsymbol{u}} \boldsymbol{u}_{I}, \quad \delta \boldsymbol{u}_{h}=\sum_{I=1}^{N_{e}} N_{I}^{\boldsymbol{u}} \delta \boldsymbol{u}_{I}, \quad \Delta \boldsymbol{u}_{h}=\sum_{I=1}^{N_{e}} N_{I}^{\boldsymbol{u}} \Delta \boldsymbol{u}_{I},
$$

with $N_{e}$ denoting the total number of nodes per element. Similarly, the numerical approximations of the phase field $d$, its variation $\delta d$ and increment $\Delta d$ as well as their spatial derivatives are expressed as

$$
\begin{gathered}
d_{h}=\sum_{I=1}^{N_{e}} N_{I}^{d} d_{I}, \quad \delta d_{h}=\sum_{I=1}^{N_{e}} N_{I}^{d} \delta d_{I}, \quad \Delta d_{h}=\sum_{I=1}^{N_{e}} N_{I}^{d} \Delta d_{I} \\
\nabla d_{h}=\sum_{I=1}^{N_{e}} \frac{\partial N_{I}^{d}}{\partial \boldsymbol{X}} d_{I}, \quad \nabla \delta d_{h}=\sum_{I=1}^{N_{e}} \frac{\partial N_{I}^{d}}{\partial \boldsymbol{X}} \delta d_{I}, \quad \nabla \Delta d_{h}=\sum_{I=1}^{N_{e}} \frac{\partial N_{I}^{d}}{\partial \boldsymbol{X}} \Delta d_{I} .
\end{gathered}
$$

Making use of the numerical approximations above and the assembly operator $\bigcup$, one can reformulate the weak forms (Eqs. 5.1 and 5.2) in the discretized form

$$
\begin{gathered}
\bigcup_{e=1}^{n_{e}} \sum_{I=1}^{N_{e}} \underline{\boldsymbol{R}}_{I, e}^{u} \cdot \delta \underline{\boldsymbol{u}}_{I}=0 \\
\bigcup_{e=1}^{n_{e}} \sum_{I=1}^{N_{e}} \delta d_{I} R_{I, e}^{d}=0
\end{gathered}
$$

with $n_{e}$ denoting the total number of elements. 
$\underline{\boldsymbol{R}}_{I, e}^{u}$ and $R_{I, e}^{d}$ are the nodal residuals which are expressed in index notation as follows

$$
\begin{aligned}
R_{I, e}^{u} & =\underbrace{\int_{\mathcal{B}_{e}} F_{r i} S_{i j} \frac{\partial N_{I}^{u}}{\partial X_{j}} \mathrm{~d} V+\int_{\partial \mathcal{B}_{e}} \rho \ddot{x}_{i} N_{I}^{u} \mathrm{~d} V}_{-P_{I, e}^{u}}-\underbrace{\int_{\partial \mathcal{B}_{N, e}} t_{i} N_{I}^{u} \mathrm{~d} A}_{-F_{I, e}^{u}} \\
& =\underbrace{R_{I, e}^{d}}_{P_{I, e}^{d}}=\underbrace{\int_{l}-2}_{\mathcal{B}_{e}-2(1-d) \mathcal{H} N_{I}^{d} \mathrm{~d} V+\int_{\mathcal{B}_{e}} \mathcal{G}_{c}\left(l \frac{\partial d}{\partial X_{i}} \cdot \frac{\partial N_{I}^{d}}{\partial X_{i}}+\frac{1}{l} d N_{I}^{d}\right) \mathrm{d} V}
\end{aligned}
$$

These residuals are computed on element level because the support of a finite element shape function $N_{I}$ usually comprises the elements adjacent to the respective node $I$. In Eqs. (5.8 and 5.9), $\underline{\boldsymbol{P}}_{I, e}^{u}$ and $P_{I, e}^{d}$ denote the contributions of internal forces, whereas $\underline{\boldsymbol{F}}_{I, e}^{\boldsymbol{u}}$ is the contribution of external mechanical loads.

The elemental residual $\underline{\boldsymbol{R}}_{I, e}$ can be obtained by assembling the mechanical residual $\underline{\boldsymbol{R}}_{I, e}^{u}$ and the phase field residual $R_{I, e}^{d}$. The subsequent assembly $\bigcup$ of all $n_{e}$ elements of the discretization yields the global residual $\underline{\boldsymbol{R}}$

$$
\underline{\boldsymbol{R}}=\bigcup_{e=1}^{n_{e}} \underline{\boldsymbol{R}}_{e} .
$$

The sum of all element contributions $\underline{\boldsymbol{R}}_{I, e}$ is denoted by the nodal residuals

$$
\underline{\boldsymbol{R}}_{I}=\sum_{e \in \mathcal{E}_{I}} \underline{\boldsymbol{R}}_{I, e}
$$

with the element $e$ being in the set $\mathcal{E}_{I}$ of elements adjacent to node $I$. A similar assembly of nodal virtual quantities $\delta \boldsymbol{u}$ and $\delta d$ is applied and gives $\delta \underline{\boldsymbol{d}}$. Thus, the global system of equations (Eqs. 5.6 and 5.7) can be expressed in the compact notation as

$$
\underline{\boldsymbol{R}} \cdot \delta \underline{\boldsymbol{d}}=0,
$$

such that Eq. (5.12) must hold for any $\delta \underline{\boldsymbol{d}}$. This results in the global system of equations

$$
\underline{\boldsymbol{R}}=\underline{\mathbf{0}} \quad \Leftrightarrow \quad \underline{\boldsymbol{R}}_{I}=\underline{\mathbf{0}} \quad \text { for } \quad I=1, \ldots, N .
$$




\subsection{Monolithic temporal discretization and iterative solution}

Algorithmically, the monolithic and staggered schemes are the approaches commonly used to solve the displacement and phase field equations (Eqs. 5.1 and 5.2). The monolithic scheme consists of solving the fully-coupled non-linear system of equations in each loading step/time-step simultaneously, whereas the staggered scheme splits the displacement field $\boldsymbol{u}$ and the phase field $d$, and solve them successively in each time step. As reported by Ambati et al. [2], the staggered approach is more robust than the monolithic one. However, it tends to underestimate the speed of the crack evolution, because the spatial system is only solved approximately and uses only small loading steps. Additionally, the monolithic scheme is more efficient as it only needs one loop of iterations. For this reason, this study considers the monolithic scheme. For a comprehensive review of these schemes, the reader is referred to the works of Borden [11] and Liu et al. [73]. This study further adopts the implicit Newmark method for the time integration scheme, as outlined in Schlüter et al. [101].

By means of the global internal and external forces $\underline{\boldsymbol{P}}$ and $\underline{\boldsymbol{F}}$, the global system of equations (Eq. 5.12) may be expressed as

$$
\underline{R}=\underline{F}-\underline{P}(\underline{d}, \underline{\dot{d}}, \underline{\ddot{d}})=\underline{0} .
$$

Herein $\underline{\boldsymbol{d}}$ represents the global vector of degrees of freedom.

In a time discretized form, where $t_{n+1}=t_{n}+\Delta t$, Eq. (5.14) takes the form

$$
\underline{\boldsymbol{R}}_{n+1}=\underline{\boldsymbol{F}}_{n+1}-\underline{\boldsymbol{P}}\left(\underline{\boldsymbol{d}}_{n+1}, \underline{\dot{\boldsymbol{d}}}_{n+1}, \ddot{\boldsymbol{d}}_{n+1}\right)=\underline{\mathbf{0}},
$$

with the subscript $n+1$ denoting the terms that are evaluated at time $t_{n+1}$.

In order to approximate the velocities $\underline{\dot{d}}_{n+1}$ and accelerations $\ddot{\ddot{d}}_{n+1}$, the implicit time integration 
scheme of Newmark [87] is utilized,

$$
\begin{array}{r}
\dot{\underline{\boldsymbol{d}}}_{n+1}=\underline{\dot{\boldsymbol{d}}}_{n}+\Delta t \underline{\ddot{\boldsymbol{d}}}_{\gamma}, \quad \text { where } \\
\ddot{\ddot{\boldsymbol{d}}}_{\gamma}=(1-\gamma) \underline{\ddot{\boldsymbol{d}}}_{n}+\gamma \ddot{\ddot{\boldsymbol{d}}}_{n+1} \\
\underline{\boldsymbol{d}}_{n+1}=\underline{\boldsymbol{d}}_{n}+\Delta t \underline{\dot{\boldsymbol{d}}}_{n}+\frac{1}{2} \Delta t^{2} \ddot{\ddot{\boldsymbol{d}}}_{\beta}, \quad \text { where } \\
\ddot{\boldsymbol{d}}_{\beta}=(1-2 \beta) \ddot{\ddot{\boldsymbol{d}}}_{n}+2 \beta \ddot{\ddot{\boldsymbol{d}}}_{n+1} .
\end{array}
$$

The parameters $\gamma$ and $\beta$ are user-defined. In this study, the particular choice $\gamma=\frac{1}{2}$ and $\beta=\frac{1}{4}$ is adopted to obtain an implicit and unconditionally stable scheme [26]. The time step size $\Delta t$ is also user-defined, but it is adapted by an automatic control in accordance to the number of iterations needed for convergence in the previous time step. The substitution of Eqs (5.16) and (5.17) into Eq. (5.15) leads to

$$
\underline{\boldsymbol{R}}_{n+1}=\underline{\hat{\boldsymbol{R}}}\left(\underline{\boldsymbol{F}}_{n+1}, \underline{\boldsymbol{d}}_{n}, \underline{\boldsymbol{d}}_{n}, \underline{\boldsymbol{d}}_{n}, \underline{\boldsymbol{d}}_{n+1}\right)=\underline{\boldsymbol{F}}_{n+1}-\underline{\hat{\boldsymbol{P}}}\left(\underline{\boldsymbol{d}}_{n}, \underline{\boldsymbol{d}}_{n}, \underline{\boldsymbol{d}}_{n}, \underline{\boldsymbol{d}}_{n+1}\right)=\mathbf{0} .
$$

As can be seen, the time discretized residual is a function of the prescribed external forces $\underline{\boldsymbol{F}}_{n+1}$, the known degrees of freedom $\underline{\boldsymbol{d}}_{n}, \underline{\boldsymbol{d}}_{n}$ and $\underline{\boldsymbol{d}}_{n}$ at time $t_{n}$, and the unknown degrees of freedom $\underline{\boldsymbol{d}}_{n+1}$ at time $t_{n+1}$.

For the purpose of finding the solution $\underline{\boldsymbol{d}}_{n+1}$ of this system of equations and due to the non-linearity of this system, the Newton-Raphson method is applied. With the initialization of $\underline{\boldsymbol{d}}_{n+1}^{(0)}=\underline{\boldsymbol{d}}_{n}$, an iterative computation of the solution is executed according to the update formula

$$
\underline{\boldsymbol{d}}_{n+1}^{(k+1)}=\underline{\boldsymbol{d}}_{n+1}^{(k)}+\Delta \underline{\boldsymbol{d}}_{n+1}^{(k)}
$$

The increment $\Delta \underline{\boldsymbol{d}}_{n+1}^{(k)}$ is obtained from the linearization of the system of equations,

$$
\underline{\boldsymbol{R}}_{n+1}^{(k+1)} \approx \underline{\boldsymbol{R}}_{n+1}^{(k)}-\underline{\boldsymbol{S}}_{n+1}^{(k)} \Delta \underline{\boldsymbol{d}}_{n+1}^{(k)}=\underline{\mathbf{0}}
$$

The superscript $k$ denotes the current Newton iteration step, with the iteration stopping whenever the residual vanishes to a prescribed level of precision. To this end, this work uses $10^{-8}$ as the residual tolerance.

$\underline{\boldsymbol{S}}_{n+1}^{(k)}$ is the overall tangent matrix of the problem, which is obtained by the derivative of the internal force vector $\underline{\hat{\boldsymbol{P}}}$ with respect to $\underline{\boldsymbol{d}}_{n+1}$. It has no contribution from the external load vector $\underline{\boldsymbol{F}}_{n+1}$ 
since the latter is not a function of the unknown nodal degrees of freedom $\underline{\boldsymbol{d}}_{n+1}$. Thus, this matrix is obtained through

$$
\underline{\boldsymbol{S}}_{n+1}^{(k)}=\frac{\partial \underline{\hat{\boldsymbol{P}}}}{\partial \underline{\boldsymbol{d}}}\left(\underline{\boldsymbol{d}}_{n}, \underline{\boldsymbol{d}}_{n}, \underline{\boldsymbol{d}}_{n}\right)
$$

Hence, the system matrix $\underline{S}_{n+1}^{(k)}$ can be expressed as

$$
\begin{gathered}
\underline{\boldsymbol{S}}_{n+1}^{(k)}=\underline{\boldsymbol{K}}^{(k)}+\underline{\boldsymbol{D}}^{(k)} \frac{\partial \underline{\dot{\boldsymbol{d}}}_{n+1}^{(k)}}{\partial \underline{\boldsymbol{d}}_{n+1}^{(k)}}+\underline{\boldsymbol{M}}^{(k)} \frac{\partial \underline{\ddot{\boldsymbol{d}}}_{n+1}^{(k)}}{\partial \underline{\boldsymbol{d}}_{n+1}^{(k)}} \\
\underline{\boldsymbol{S}}_{n+1}^{(k)}=\underline{\boldsymbol{K}}^{(k)}+\frac{\gamma}{\beta \Delta t} \underline{\boldsymbol{D}}^{(k)}+\frac{1}{\beta \Delta t^{2}} \underline{\boldsymbol{M}}^{(k)},
\end{gathered}
$$

with the global stiffness matrix

$$
\underline{\boldsymbol{K}}^{(k)}=\frac{\partial \underline{\boldsymbol{P}}}{\partial \underline{\boldsymbol{d}}_{n+1}^{(k)}}
$$

the global damping matrix

$$
\underline{\boldsymbol{D}}^{(k)}=\frac{\partial \underline{\boldsymbol{P}}}{\partial \underline{\dot{\boldsymbol{d}}}_{n+1}^{(k)}}=\underline{\mathbf{0}},
$$

and the global mass matrix

$$
\underline{\boldsymbol{M}}^{(k)}=\frac{\partial \underline{\boldsymbol{P}}}{\partial \underline{\boldsymbol{d}}_{n+1}^{(k)}} .
$$

As can be seen in Eq. (5.24), the damping matrix $\underline{\boldsymbol{D}}^{(k)}$ is not considered in this study as it is assumed that all the energy dissipates from the system only due to fracture. The matrices are computed for each finite element separately and then assembled to the global matrices.

The local stiffness matrix $\underline{\boldsymbol{K}}_{I J, e}^{(k)}$,

$$
\underline{\boldsymbol{K}}_{I J, e}^{(k)}=\left[\begin{array}{ll}
\underline{\boldsymbol{K}}_{I J, e}^{\boldsymbol{u u}} & \underline{\boldsymbol{K}}_{I J, e}^{\boldsymbol{u d}} \\
\underline{\boldsymbol{K}}_{I J, e}^{d \boldsymbol{u}} & \underline{\boldsymbol{K}}_{I J, e}^{d d}
\end{array}\right]
$$

is obtained by the derivation of the internal force $\underline{\boldsymbol{P}}_{I, e}^{\boldsymbol{u}}$ and $P_{I, e}^{d}$ with respect to $\underline{\boldsymbol{u}}_{J}$ and $d_{J}$. The 
different elements of this matrix in index notation are

$$
\begin{aligned}
K_{I J, e}^{u u} & =\int_{\Omega}\left(S_{i j} \frac{\partial N_{J}^{u}}{\partial X_{i}} \frac{\partial N_{I}^{u}}{\partial X_{j}}+2 H_{i j k l} F_{s k} \frac{\partial N_{J}^{u}}{\partial X_{l}} F_{r i} \frac{\partial N_{I}^{u}}{\partial X_{j}}\right) \mathrm{d} V \\
K_{I J, e}^{u d} & =\int_{\Omega} \frac{\partial S_{i j}}{\partial d} N_{J}^{d} F_{r i} \frac{\partial N_{I}^{u}}{\partial X_{j}} \mathrm{~d} V \\
K_{I J, e}^{d u} & =\int_{\Omega}\left(-2(1-d) \frac{\partial \mathcal{H}}{\partial E_{i j}} F_{r i} \frac{\partial N_{J}^{u}}{\partial X_{j}} N_{I}^{d}\right) \mathrm{d} V \\
K_{I J, e}^{d d} & =\int_{\Omega}\left(\left(2 \mathcal{H}+\frac{\mathcal{G}_{c}}{l}\right) N_{J}^{d} N_{I}^{d}+\mathcal{G}_{c} l \frac{\partial N_{J}^{d}}{\partial X_{i}} \frac{\partial N_{I}^{d}}{\partial X_{j}}\right) \mathrm{d} V .
\end{aligned}
$$

As $\underline{\boldsymbol{P}}_{I, e}^{u}$ and $P_{I, e}^{d}$ are not a function of $\underline{\ddot{\boldsymbol{u}}}_{J}$ and $\ddot{d}_{J}$, the local mass matrix $\underline{\boldsymbol{M}}_{I J, e}^{(k)}$ takes the simplified form

$$
\underline{M}_{I J, e}^{(k)}=\left[\begin{array}{cc}
\underline{M}_{I J, e}^{u u} & \underline{\mathbf{0}} \\
\underline{\mathbf{0}} & \underline{\mathbf{0}}
\end{array}\right],
$$

with

$$
\underline{\boldsymbol{M}}_{I J, e}^{u u}=\frac{\partial \underline{\boldsymbol{P}}_{I, e}^{u}}{\partial \underline{\ddot{\boldsymbol{u}}}_{J}}=\int_{\mathcal{B}_{e}} N_{I} \rho_{0} \frac{\partial \underline{\ddot{\boldsymbol{u}}}_{h}}{\partial \underline{\ddot{\boldsymbol{u}}}_{J}} \mathrm{~d} V \mathbf{1}=\int_{\mathcal{B}_{e}} N_{I} \rho_{0} N_{J} \mathrm{~d} V \mathbf{1}
$$

In accordance with Eqs (5.22) and (5.24), the element system (tangent) matrix can be simplified to

$$
\underline{\boldsymbol{S}}_{I J, e}^{(k)}=\underline{\boldsymbol{K}}_{I J, e}^{(k)}+\frac{1}{\beta \Delta t^{2}} \underline{\boldsymbol{M}}_{I J, e}^{(k)} .
$$

The assembly of all element system (tangent) matrices yields the global system (tangent) matrix

$$
\underline{\boldsymbol{S}}_{n+1}^{(k)}=\bigcup_{e=1}^{n_{e}} \underline{\boldsymbol{S}}_{e}^{(k)}
$$




\section{Chapter 6}

\section{Numerical examples}

This chapter evaluates the performance of the dynamic phase field model implemented in chapter 5 . In this regard, two representative three-dimensional problems are solved using the neo-Hookean material law introduced in section 2.5 and the anisotropic split discussed in subsection 4.2.2. These problems include the benchmark tests of tension and shear applied to a single-edge notched block. These examples aim to demonstrate that the coupled motion and phase field equations (Eqs 5.1 and $5.2)$ entirely determine the propagation of cracks. Furthermore, this chapter investigates the effect of the length-scale parameter $l$ and mesh refinement level on the results of simulations. However, this investigation does not include simulations with very small values of the length scale, which are associated with fine mesh sizes, due to the time limitations of this study. Thus, the results of this investigation aim to only ascertain the trends due to the length-scale parameter and mesh level.

For all the problems discussed in this chapter, a pre-existing crack is used to initialize the crack propagation. Several approaches can be used to model initial cracks. In this study, a discrete crack is introduced in the geometry. An alternative approach is to explicitly induce a crack in the domain through the use of the phase field. The value of the latter is prescribed to the fully broken state, as presented by Schlüter et al. [101]. Another approach, which was proposed by Borden [11], uses an initial strain-history field. This approach allows locating initial cracks anywhere in the domain without reference to the mesh. These alternative approaches can be considered in future studies.

With regards to the spatial discretization, this work applies a mesh refinement locally to better capture cracks and to alleviate the high computational costs associated with simulations of phase field models. With the a priori knowledge of the crack path, elements are refined within the areas 
of expected crack propagation. The pre- and post-processing commercial $\mathrm{GiD}^{1}$ is used for the problem definition, mesh generation, and solution visualization. However, strategies of adaptive mesh refinement are not considered in this work.

The size of the length-scale parameter $l$ can be chosen based on the effective mesh size $h$ or the smallest dimension $L_{s}$ of a model. In the former, Miehe et al. [78], Schlüter et al. [101] and Ambati et al. [2] reported that the length-scale parameter from Eq. (4.9) for the width of the diffusive crack area should be at least twice larger than the effective mesh size to resolve the smooth crack with sufficient accuracy. Thus the ratio $l \geq 2 h$ is defined as the accuracy condition in this work. In the latter choice, Amor et al. [5] established an empirical relation using the smallest dimension of the model to cap the maximum value of the length-scale parameter for a stable crack analysis. This

relation is given by $l \leq \frac{L_{s}}{100}$. Hence, combining the stability relation of Amor et al. [5] with the accuracy ratio for the length-scale would require extremely small mesh sizes, which demands significant computational time and memory. Considering that this study aims to investigate the qualitative effects of the length-scale parameter on the results of simulations and due to time constraints, the choice of the length-scale parameter does not meet this stability criterion.

In addition to the use of the implicit Newmark time integration scheme discussed in section 5.2 , fracture modeling requires very small time steps because the phase field changes rapidly over the diffusive area. In this work, an initial time increment of $t=10^{-7} \mathrm{~s}$ is considered appropriate with an automatic step size control.

\subsection{Single-edge notched block under tension}

The geometry of this problem consists of a single-edge notched block of dimensions of $1 \times 1 \times 0.1 \mathrm{~m}$ and notch length $0.5 \mathrm{~m}$, which is depicted in Figure 6.1. This depiction also shows the boundary conditions applied to this problem. The upper boundary has an incrementally increasing displacement condition, which is applied at the initial time step, pointing in the positive y-direction such that the crack growth occurs, generally with increasing speed [108]. The lower boundary is completely constrained with $u_{x}=u_{y}=u_{z}=0$. All the other boundaries are free of Dirichlet boundary conditions to prevent the rotation of the block and maintain its symmetry. This problem corresponds to the classical fracture mode I introduced in section 3.1.

\footnotetext{
${ }^{1}$ More details on the commercial software GiD are provided in https://www.gidhome.com.
} 


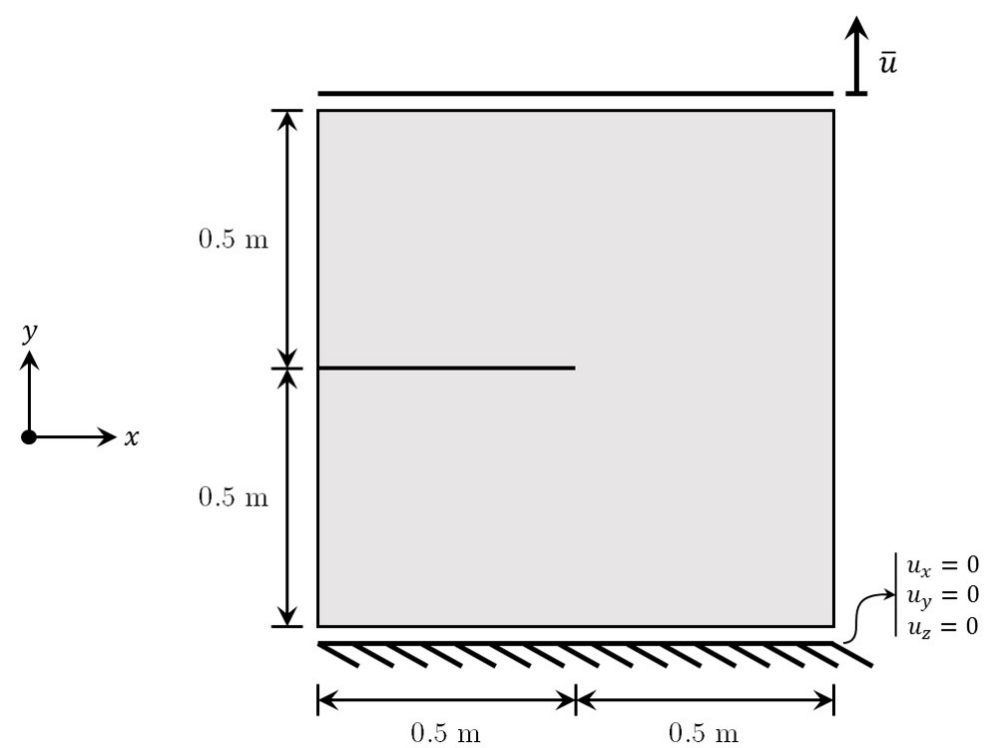

Figure 6.1: Problem definition of a single-edge notched block under tension.

The model parameters of this problem are given in Table 6.1.

Table 6.1: Model parameters for the single-edge notched block under tension.

\begin{tabular}{|c|c|c|c|c|c|}
\hline Parameters & Values & Units & Dimensions & Values & Units \\
\hline Shear modulus $\mu$ & $80.769 \times 10^{9}$ & $\mathrm{~N} / \mathrm{m}^{2}$ & Length $L$ & 1 & $\mathrm{~m}$ \\
\hline Bulk modulus $\kappa$ & $175 \times 10^{9}$ & $\mathrm{~N} / \mathrm{m}^{2}$ & Height $H$ & 1 & $\mathrm{~m}$ \\
\hline Numerical parameter $k$ & 0.001 & - & Thickness $T$ & 0.1 & $\mathrm{~m}$ \\
\hline Crack resistance $\mathcal{G}_{c}$ & 2700 & $\mathrm{~J} / \mathrm{m}^{2}$ & Notch length $l$ & 0.5 & $\mathrm{~m}$ \\
\hline Density $\rho$ & 1000 & $\mathrm{~kg} / \mathrm{m}^{3}$ & Notch thickness $t_{\mathrm{n}}$ & $2.0 \times 10^{-3}$ & $\mathrm{~m}$ \\
\hline
\end{tabular}

With the a priori knowledge of the crack path, a local refinement mesh is applied within the area of expected crack growth. In this tension test, three study cases are considered to investigate the effects which arise due to the choice of the length-scale parameter. All these cases use the same mesh but have different ratios of the length-scale to the effective mesh size. In this regard, the accuracy condition is met for all the ratios with $l=5 h, l=3 h$, and $l=2 h$ for case 1,2 and 3 , respectively. Table 6.2 summarizes the study parameters used for each case. 
Table 6.2: Study parameters for single-edge notched block under tension.

\begin{tabular}{|l|c|c|c|c|c|c|c|}
\cline { 2 - 8 } \multicolumn{1}{c|}{} & $\begin{array}{c}\text { Length- } \\
\text { scale } \\
l(\mathrm{~m})\end{array}$ & $\begin{array}{c}\text { Mesh size of } \\
\text { line elements } \\
\text { away from } \\
\text { the crack } \\
h_{a}\end{array}$ & $\begin{array}{c}\text { Mesh size of } \\
\text { line elements } \\
\text { within the } \\
\text { expected } \\
\text { crack area } \\
h\end{array}$ & $\begin{array}{c}\text { Linear } \\
\text { elements }\end{array}$ & $\begin{array}{c}\text { Triangular } \\
\text { elements }\end{array}$ & $\begin{array}{c}\text { Tetrahedral } \\
\text { elements }\end{array}$ & $\begin{array}{c}\text { Number } \\
\text { of } \\
\text { nodes }\end{array}$ \\
\hline Case 1 & 0.05 & 0.1 & 0.01 & 317 & 2284 & 5471 & 9606 \\
\hline Case 2 & 0.03 & 0.1 & 0.01 & 317 & 2284 & 5471 & 9606 \\
\hline
\end{tabular}

Regarding the local refinement in the area of the expected crack path, Figure 6.2 depicts the mesh used for the test cases.
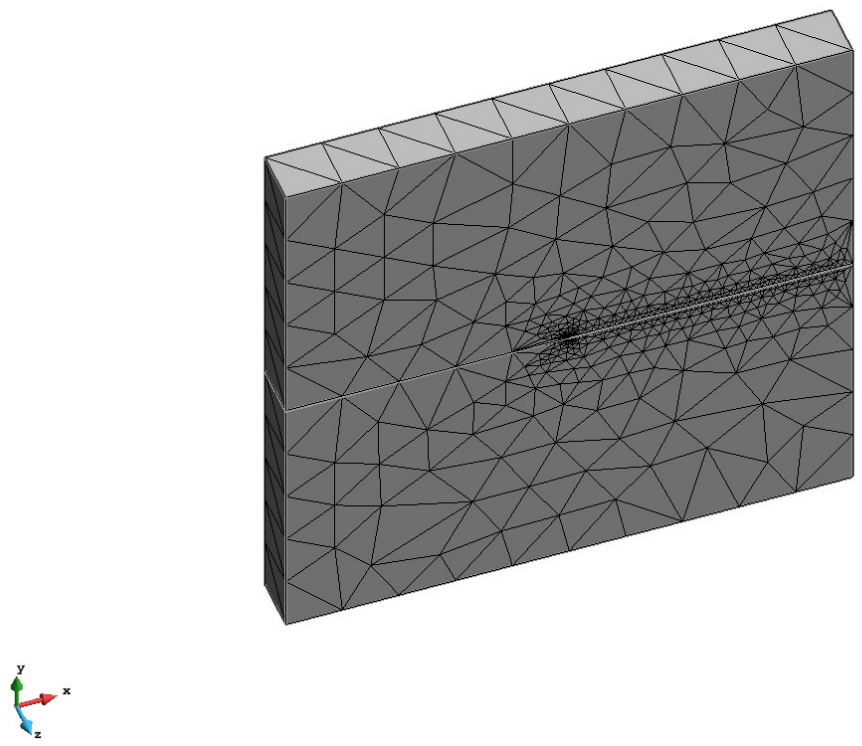

Figure 6.2: Generated mesh of single-edge notched block under tension.

For a similar problem, Hesch et al. [45] presented the phase field contour in Figure $6.3_{(\mathbf{b})}$. Departing from the problem in this study, they used a fourth-order phase field approximation functional, a cubic degradation function, a different anisotropic split and a Mooney-Rivlin model with a thickness of $0.2 \mathrm{~m}$. A finite element mesh was also locally refined and resulted in 46288 elements with 275 354 nodes. Despite these differences, this contour plot can be used for a qualitative comparison of the crack path as it is in agreement with several findings in literature; see Miehe et al. [78], Borden [11], Ambati et al. [2] and Steinke and Kaliske [110]. 


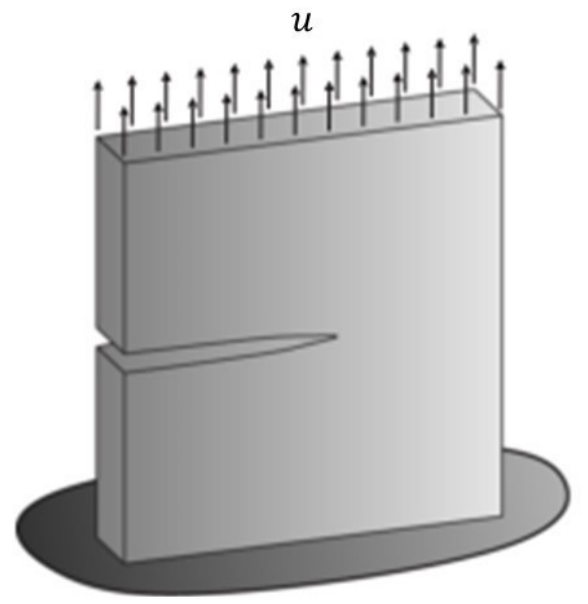

(a)

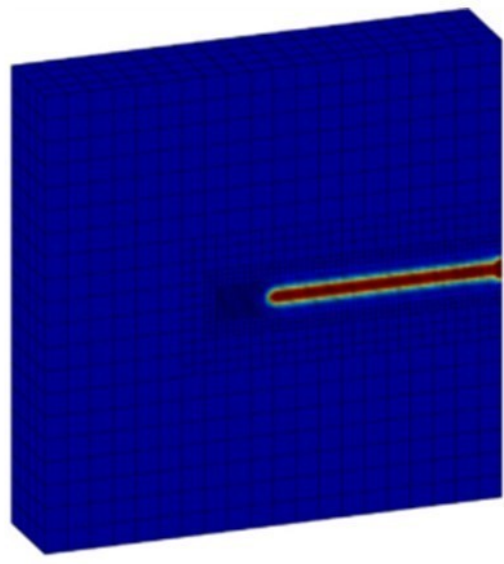

(b)

Figure 6.3: (a) Boundary conditions for tension case used in Hesch et al. [45]. (b) Phase field contour plot for tension case obtained by Hesch et al. [45].

For the tension test in this study, the following contour plots of the phase field for cases 1,2 and 3 were obtained.

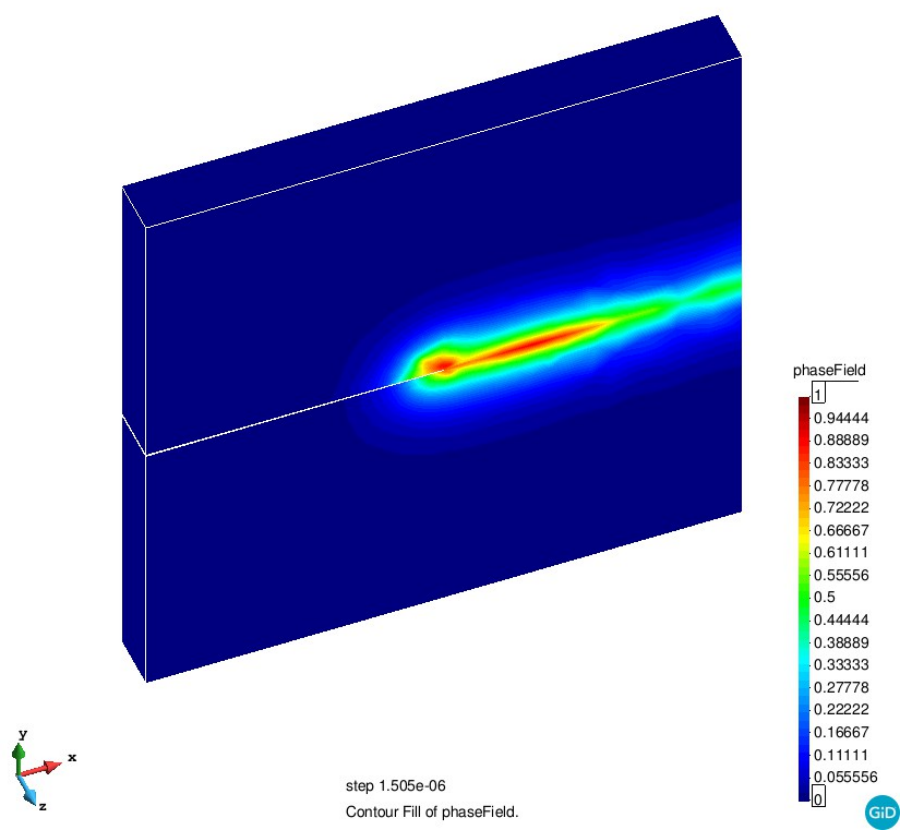

Figure 6.4: Phase field contour plot for tension case 1 at $l=0.05 \mathrm{~m}$. 


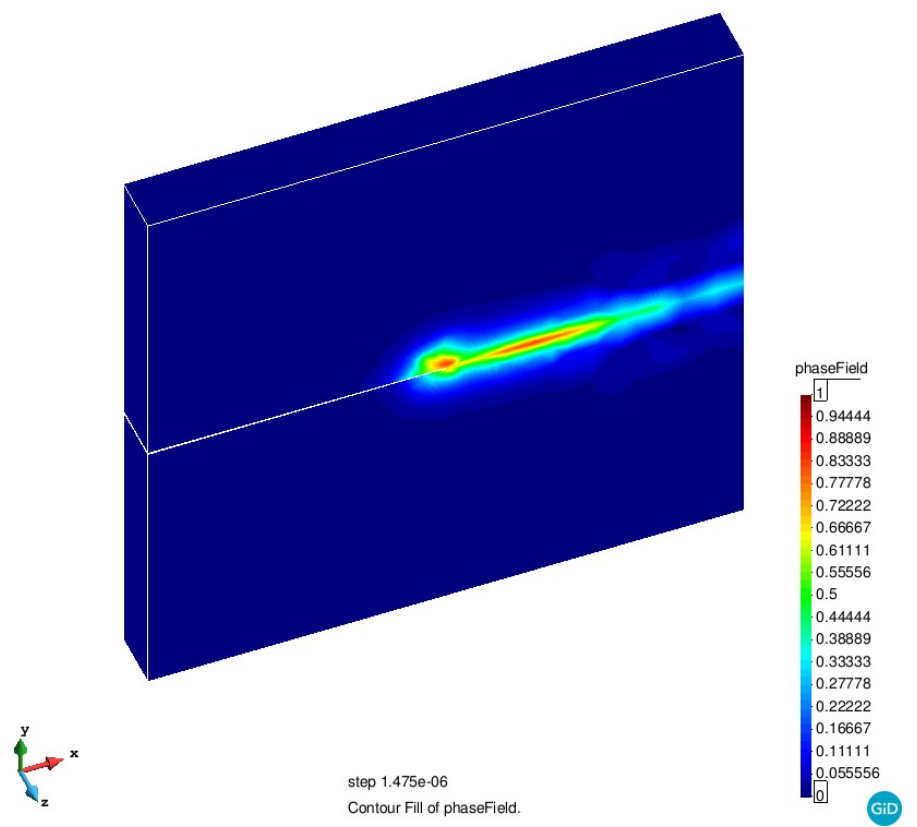

Figure 6.5: Phase field contour plot for tension case 2 at $l=0.03 \mathrm{~m}$.

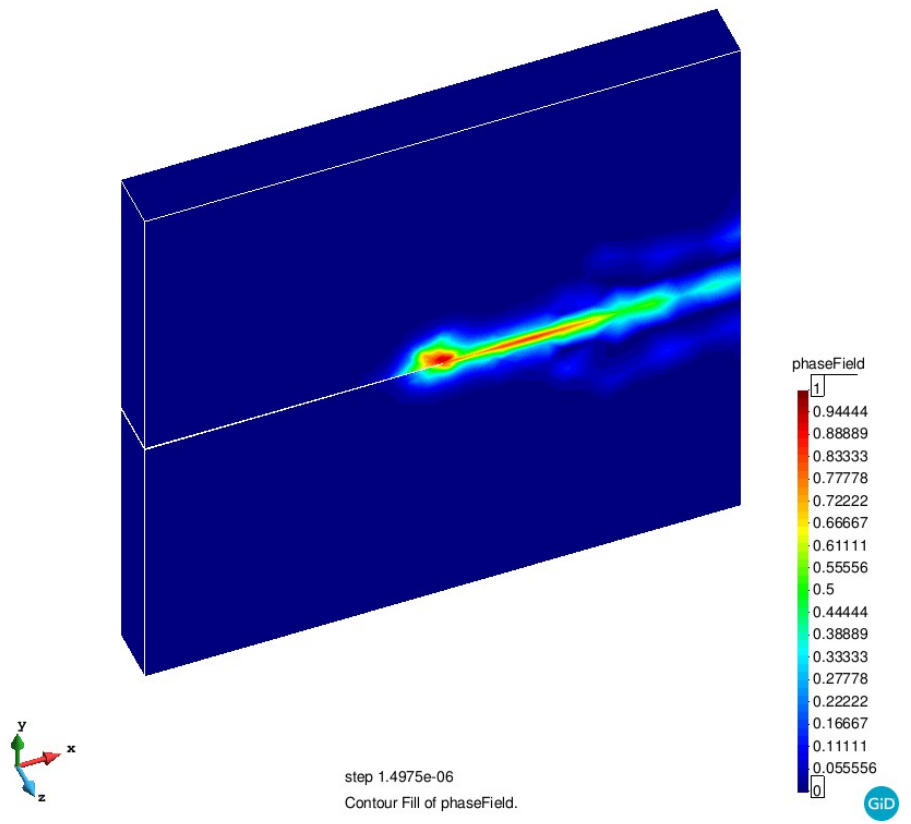

Figure 6.6: Phase field contour plot for tension case 3 at $l=0.02 \mathrm{~m}$. 
The contour plots in Figure 6.4, Figure 6.5 and Figure 6.6 show the crack patterns at different length-scales. In all plots, the crack propagates along the expected crack path towards the middle right edge of the specimen. The direction of crack propagation is in agreement with the plot of Hesch et al. [45] in Figure 6.3(b) .

Furthermore, these plots illustrate that the length-scale parameter governs the size of the diffusive area, as mentioned in section 4.1. As the parameter decreases, the diffusive area decreases. One can observe that the sharpest crack pattern is obtained for the smallest length-scale parameter $l=0.02$ m. Thus, smaller length-scale parameters yield sharper crack topologies. This observation agrees with the expected impact of the length-scale parameter on the crack topology.

Additionally, these plots show that the propagation of cracks is hampered and does not reach the right edge of the block, as expected. One reason for this effect is that the mesh used in this section is relatively too coarse. On this subject, Schlüter et al. [101] reported that a too coarse mesh can not only strongly influence the crack path but can also restrict its propagation. Borden et al. [12] further reported that this restriction results in the overestimation of the bulk energy. Thus, the size of elements must be sufficiently small. However, smaller mesh sizes are not investigated in this work due to time constraints, but they can be considered in future studies.

The results of the tension test are also provided at a notch point using the graphs in Figure 6.7 and Figure 6.8. The former figure is in terms of the Cauchy stress $\boldsymbol{\sigma}_{y y}$ (Eq. 2.18) and Almansi strain $\boldsymbol{e}_{y y}$ (Eq. 2.12), while the latter shows the relationship between the Cauchy stress $\boldsymbol{\sigma}_{y y}$ (Eq. 2.18) and length-scale parameter $l$.

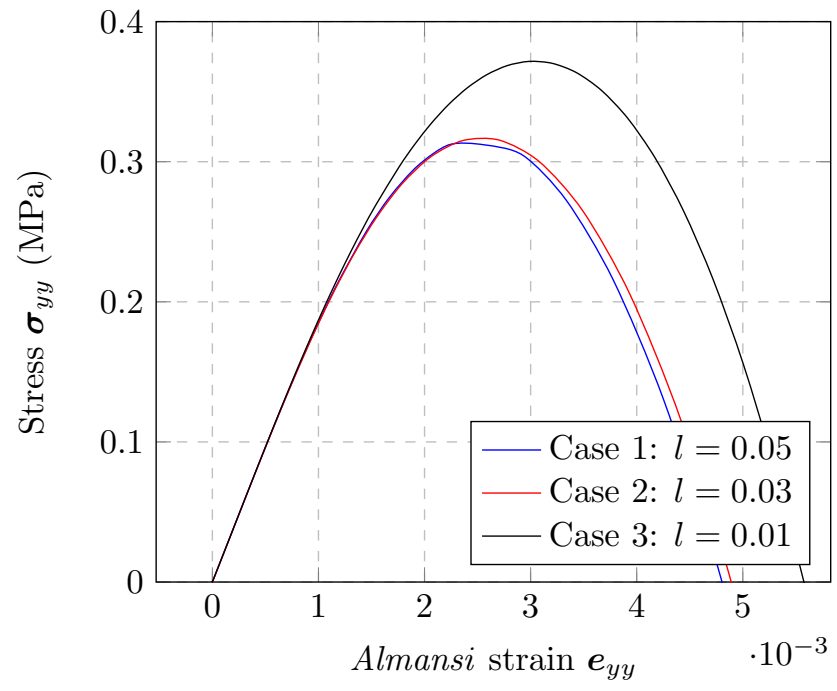

Figure 6.7: Stress $\boldsymbol{\sigma}_{y y}$ vs. Almansi strain $\boldsymbol{e}_{y y}$ at notch point for cases of block under tension. 


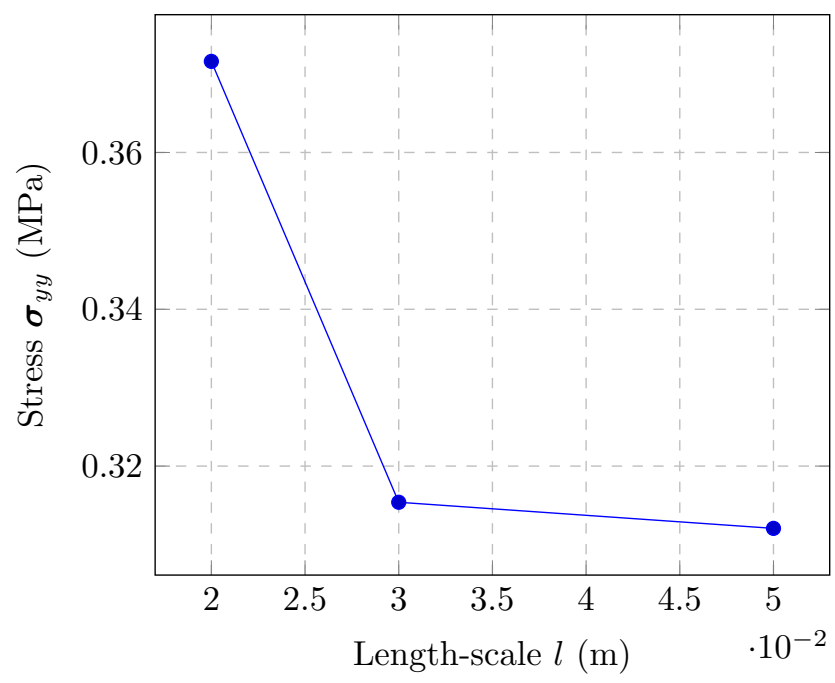

Figure 6.8: Stress $\boldsymbol{\sigma}_{y y}$ vs. Length-scale $l$ at notch point for cases of block under tension.

Figure 6.7 shows that the system response tends to be linear until the occurrence of brittle crack growth, which induces a post-peak softening branch. Figure 6.8 further indicates that the critical stress (failure stress) of the system depends on the length-scale. In this regard, this stress increases with reduced length-scales. It is the largest at the smallest length-scale $l=0.02 \mathrm{~m}$. These results are consistent with the findings reported by Amor et al. [5], Nguyen et al. [88] and Paggi and Reinoso [89]. 


\subsection{Single-edge notched block under shear}

This problem consists of a pure shear test and has the same model parameters (Table 6.1) and boundary conditions as that of the tension test above. The only departing condition from the tension test is that the non-zero driving displacement is applied pointing towards the positive $x$ direction, as shown in Figure 6.9. Thus, this problem is the classical fracture mode II introduced in section 3.1.

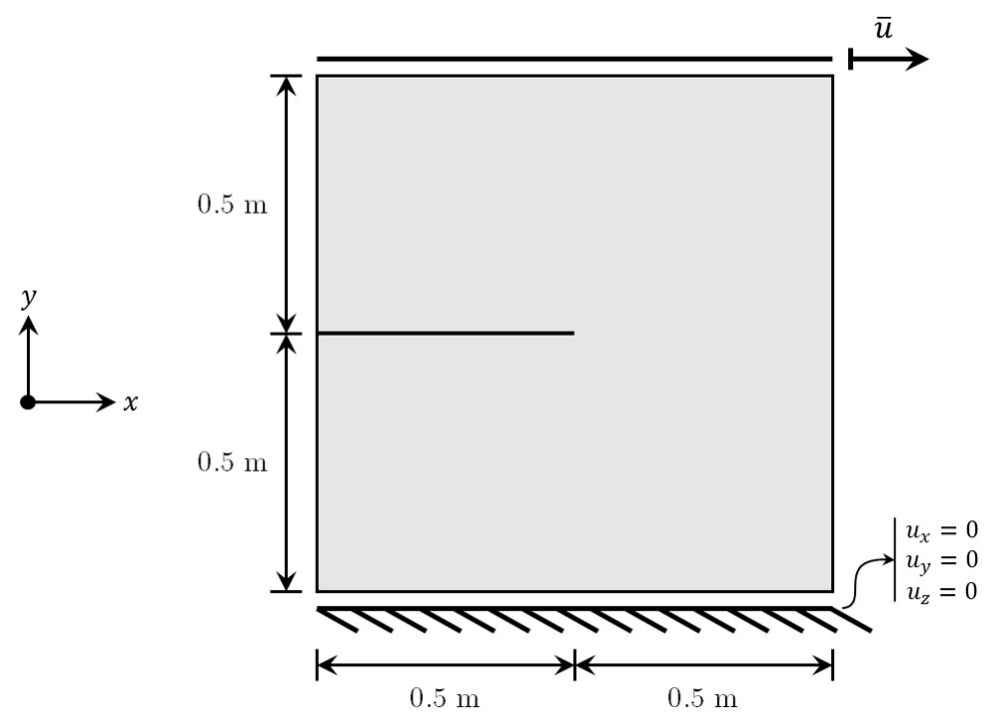

Figure 6.9: Geometry and boundary conditions of single-edge notched block under shear.

Following the a priori knowledge of the crack path, a local refinement mesh is also applied within the area of expected crack growth as shown in Figure 6.10. 

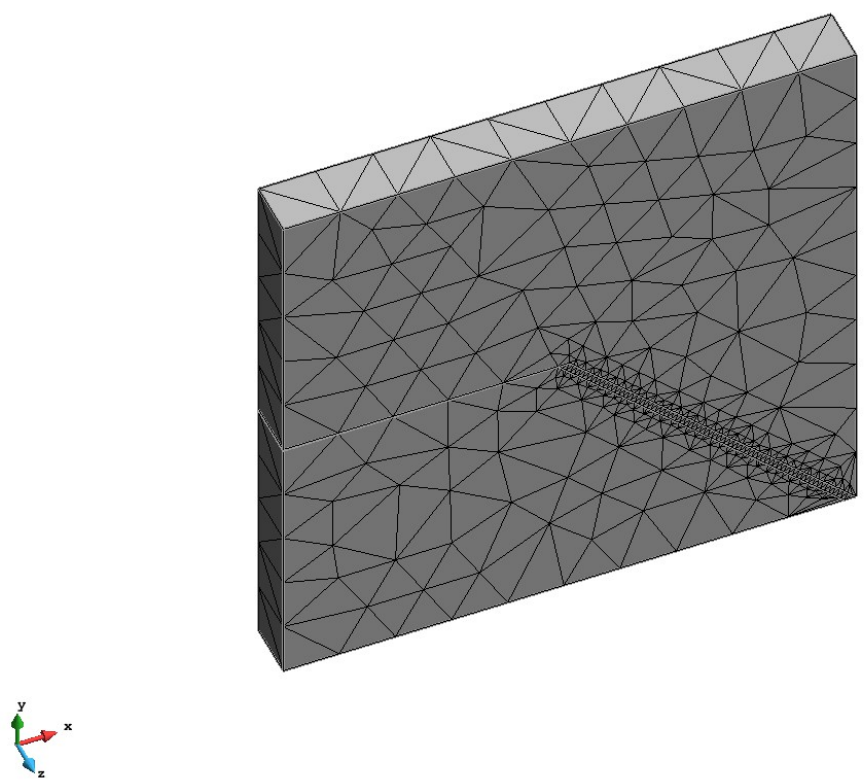

Figure 6.10: Generated mesh of single-edge notched block under shear.

Three test cases are considered in this section for the discussion on the effect of the length-scale $l$ and mesh size $h$ on the solutions. The length-scale of case 1 is chosen such that the accuracy condition is not met with $l=h$. This choice aims to show that the size of the mesh and length-scale could yield unrealistic results. On the other hand, the length-scale in cases 2 and 3 meet the accuracy condition as $l=5 h$ and $l=2 h$, respectively. The study parameters for each case are summarized in Table 6.3.

Table 6.3: Study parameters for single-edge notched block under shear.

\begin{tabular}{|c|c|c|c|c|c|c|c|}
\cline { 2 - 9 } \multicolumn{1}{c|}{} & $\begin{array}{c}\text { Length- } \\
\text { scale } \\
l(\mathrm{~m})\end{array}$ & $\begin{array}{c}\text { Mesh size } \\
\text { away from } \\
\text { the crack } \\
h_{a}\end{array}$ & $\begin{array}{c}\text { Mesh size } \\
\text { within the } \\
\text { expected } \\
\text { crack area } \\
h\end{array}$ & $\begin{array}{c}\text { Linear } \\
\text { elements }\end{array}$ & $\begin{array}{c}\text { Triangular } \\
\text { elements }\end{array}$ & $\begin{array}{c}\text { Tetrahedral } \\
\text { elements }\end{array}$ & $\begin{array}{c}\text { Number } \\
\text { of } \\
\text { nodes }\end{array}$ \\
\hline Case 1 & 0.10 & 0.1 & 0.10 & 141 & 590 & 750 & 1640 \\
\hline Case 2 & 0.05 & 0.1 & 0.01 & 397 & 1788 & 4533 & 7868 \\
\hline Case 3 & 0.02 & 0.1 & 0.01 & 397 & 1788 & 4533 & 7868 \\
\hline
\end{tabular}

Similar to the tension test above, Hesch et al. [45] presented the phase field contour result shown in Figure $6.11_{(\mathbf{b})}$ for the shear test. In this test, the mesh consisted of 21900 elements with 102807 nodes. This contour plot also corresponds qualitatively to several findings with respect to the crack propagation being towards the bottom right corner; see Miehe et al. [78], Borden [11], Ambati et al. 
[2] and Steinke and Kaliske [110].

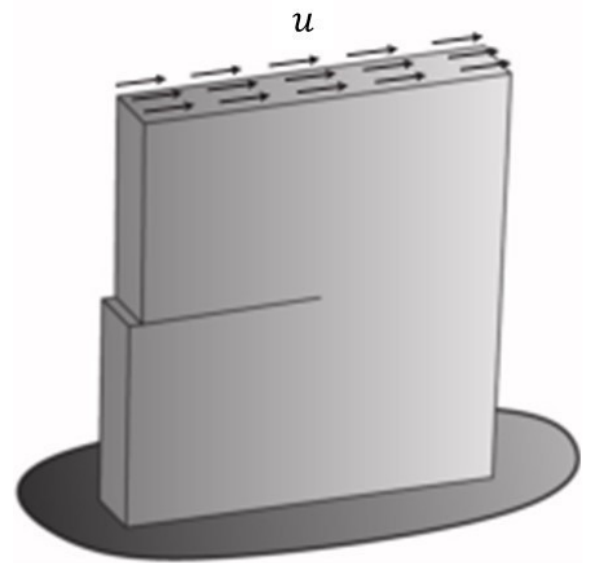

(a)

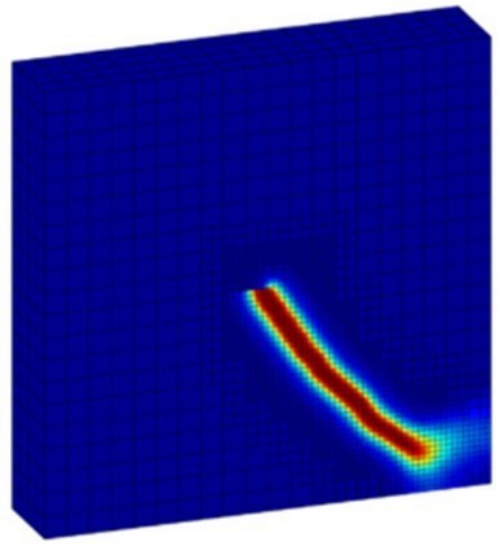

(b)

Figure 6.11: (a) Boundary conditions for shear case used in Hesch et al. [45]. (b) Phase field contour plot for shear case obtained by Hesch et al. [45].

For the shear test in this thesis, the following contour plots of the phase field were obtained for cases 1,2 and 3.

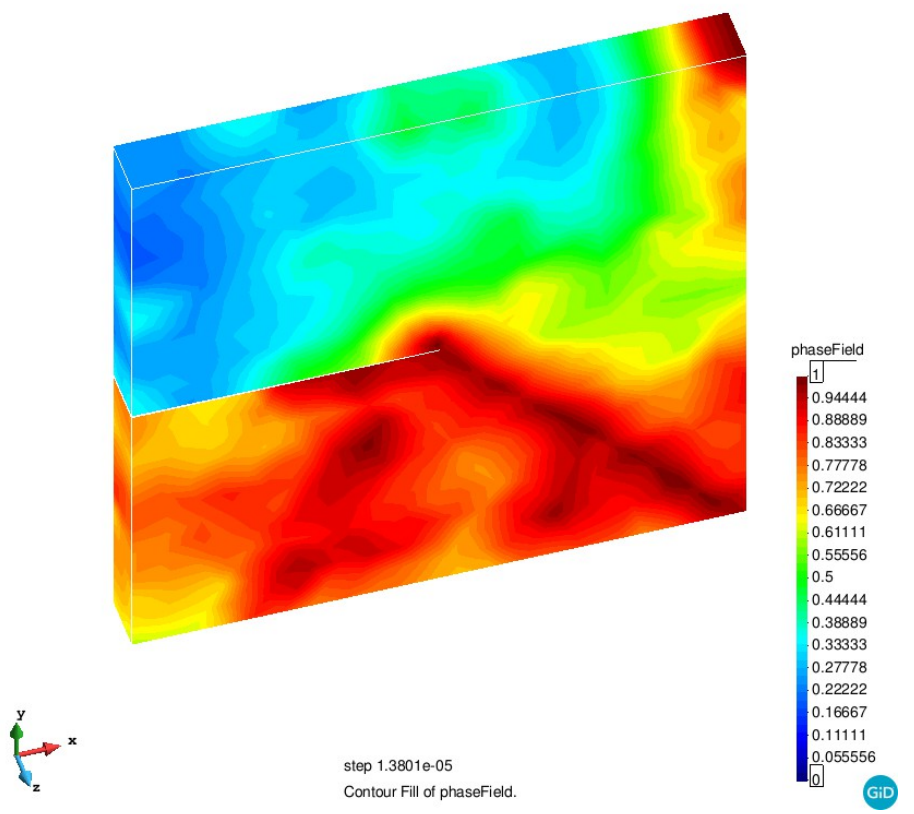

Figure 6.12: Phase field contour plot for shear case 1 at $l=0.10 \mathrm{~m}$. 


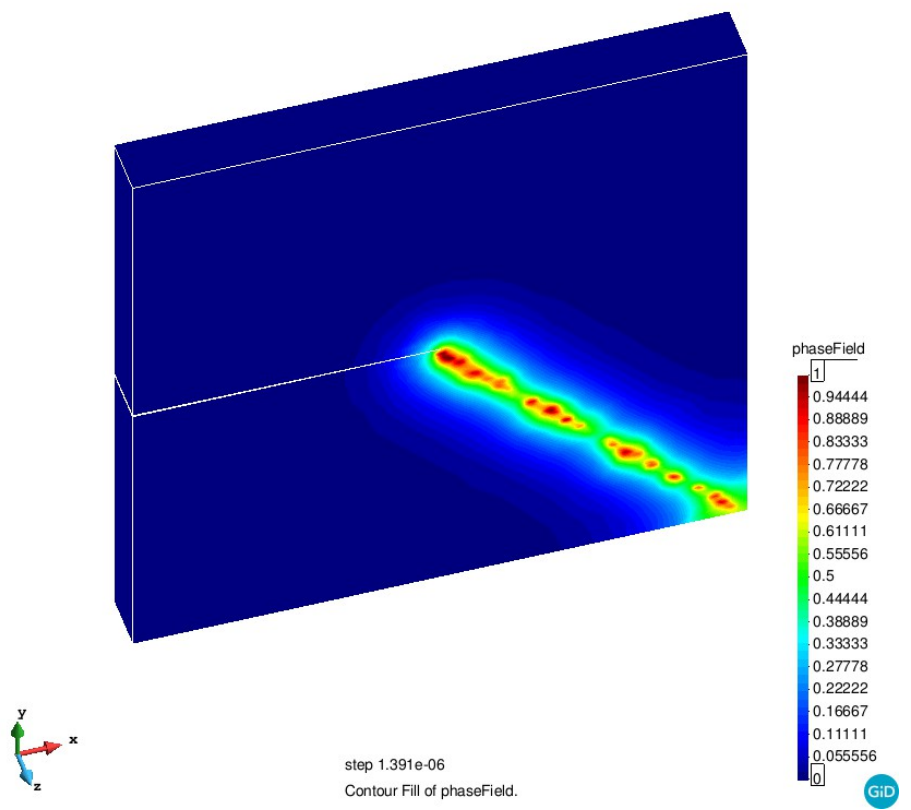

Figure 6.13: Phase field contour plot for shear case 2 at $l=0.05 \mathrm{~m}$.

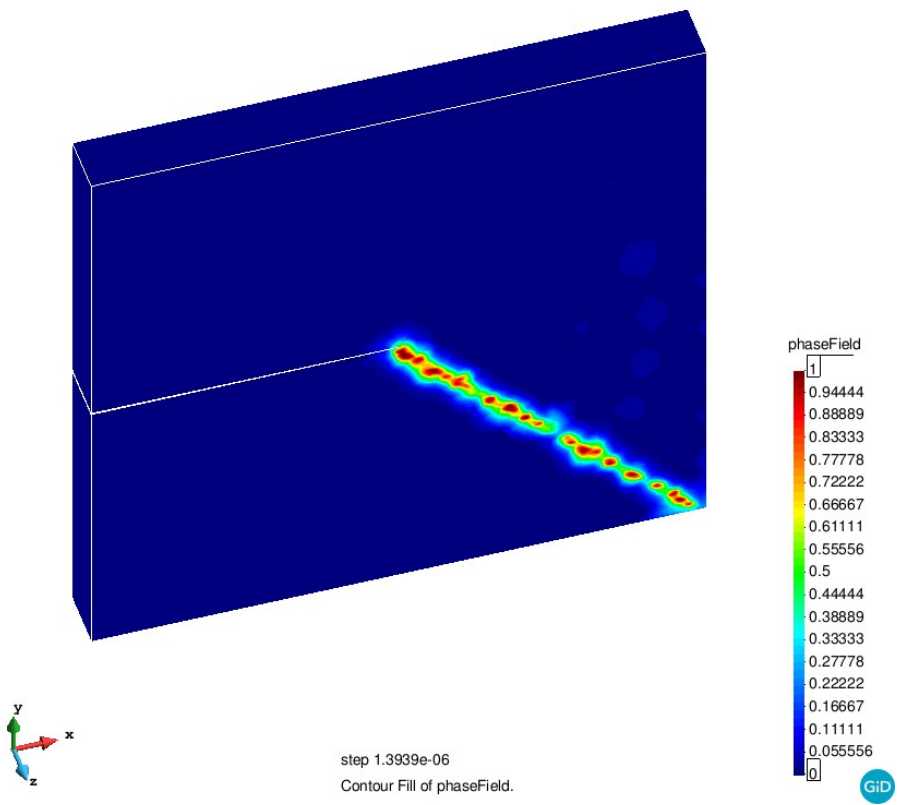

Figure 6.14: Phase field contour plot for shear case 3 at $l=0.02 \mathrm{~m}$. 
These contour plots above further show the influence of the length-scale parameter on the solutions. Figure 6.12 shows the results obtained using a ratio of the length-scale to the mesh size not meeting the accuracy condition $l \geq 2 h$. In this case, the contour plot provides unrealistic solutions. The latter is caused by the ratio of the length-scale to the mesh size $l=h$ and their large values. This case is purposely provided to demonstrate that large values of the length-scale and mesh size cannot only hamper the crack propagation but can also provide physically unrealistic results. This finding is consistent with the conclusions reported by Pundir [93]. Due to time constraints, further investigation of the effect of small values of the length-scale and mesh size on the crack topology and propagation are reserved for future works.

In contrast to Figure 6.12, Figure 6.13 and Figure 6.14 show the expected crack topology, albeit not the sharpest crack topology. These plots confirm that a small length-scale converges to the sharp crack topology, as reported by Miehe et al. [78], Borden [11] and Ambati et al. [2]. Additionally, the plots show that the mesh size also affects the accuracy of the solutions. In this regard, the crack does not completely evolve down to the bottom edge of the block, and the propagation of the crack is not entirely continuous through the crack path. Thus, fine mesh sizes associated with small length-scales would provide a sharp crack topology that could extend to the bottom face.

Furthermore, the effect of the local mesh refinement is apparent when comparing the results in Figure 6.12, Figure 6.13 and Figure 6.14 to those of Hesch et al. [45] in Figure $6.11_{(\mathbf{b})}$. One can see that the refinement area in this work differs with the one of Hesch et al. [45]. As a result, the crack path differs. This difference is expected because crack propagates in locally refined areas. Thus, the choice of the refinement area is valid in cases where the expected crack path is known. For cases where the crack path is hard to predict, adaptive refinement strategies can be considered [48]. 


\section{Chapter 7}

\section{Conclusions and future work}

The primary objective of this study was to implement a phase field model for dynamic brittle fracture at finite strains and to investigate the effect of the length-scale parameter and mesh size on the results of simulations. To this end, two representative problems were solved, and their results discussed. This chapter summarises the significant findings, acknowledge any shortcomings and outline future studies.

\subsection{Conclusions}

This thesis addressed the modeling of dynamic brittle fracture at finite strains using the phase field method proposed by Miehe and Schänzel [77]. One attribute of this model is that it smoothly represents a crack and does not require the algorithmic treatment of the crack growth. Before addressing the phase field model, this study reviewed relevant concepts of classical continuum mechanics and definitions of fracture mechanics. Subsequently, the discussion on the phase field method started with the approximation of a sharp crack topology first in one dimension and then generalized to two and three dimensions.

This work further outlined the decomposition of the bulk energy to account for the different fracture behavior in tension and compression. This decomposition aims to prevent the crack growth due to compression and to model crack closure. Hereof, two methods, the isotropic split and anisotropic split based on the volumetric-deviatoric decomposition of Amor et al. [5], were outlined. The first method allows the crack to grow under tension, shear and compression, while the second assumes 
that crack propagates as a result of volumetric expansion and deviatoric deformation but not of volumetric compression. Furthermore, the irreversibility of the fracture process was modeled by a local history field. The latter ensures that the maximum bulk energy during the full process history determines the present stiffness. This condition, in turn, prevents crack healing from occurring.

The variational formulation of the phase field model was herein discussed. Additionally, the equation of motion and the evolution equation of the phase field were explicitly provided. Due to the nonlinearity of the coupled system of equations, the Newton-Raphson method was used to solve this system while controlling the time step size automatically. The implementation of this model was carried out using a monolithic scheme within the in-house software SESKA. This scheme consists of computing the displacement and phase field simultaneously. Furthermore, the Newmark time integration scheme was employed.

After the implementation of the phase field model, this work evaluated the performance of the model and investigated the effect of the length-scale parameter and mesh size on the results of simulations by solving the tension and shear tests on a single-edge notched block. A local refinement was further applied in the area of expected crack growth to reduce the rather high computational costs associated with phase field models, and no adaptive remeshing technique was employed. Additionally, small values of the length-scale, which are associated with extremely fine mesh, were not considered for simulations due to time limitations. Thus, the investigation only aims to ascertain the trend due to the length-scale parameter and mesh size.

The results of the investigation showed that the dynamic phase field model could reproduce the propagation of cracks without any additional algorithmic treatment. Furthermore, it was observed that the choice of the length-scale and mesh size influences the results. As expected, a small value of the length-scale converges to the sharp crack topology. In contrast, a large value of the length-scale combined with a too coarse mesh size can yield unrealistic results. Thus, the length-scale parameter should be chosen sufficiently small in conjunction with the mesh size to obtain accurate results. Additionally, the length-scale also influences the failure stress. On this point, a small value of the length-scale yields a larger stress value. 


\subsection{Future work}

This work is part of a project aimed at the modeling of Antarctic sea ice dynamics. One aspect of this project is the modeling of the gradual break-up of the consolidated ice in spring. In this regard, the contribution of this work is the first step in addressing this aspect. Due to time constraints, this thesis only focused on the implementation of the phase field model of dynamic brittle fracture at finite strains and investigated the effect of the length-scale parameter and mesh size on results of simulations. Thus, several studies can be suggested for future work. These studies can be summarized in a two-pronged approach provided below. Note that the list of future studies presented below is by no means exhaustive, but gives an indication of future studies to consider.

\subsubsection{Extension of the implemented phase field model for dynamic frac- ture}

This first approach aims to extend the implemented phase field model with the following proposed future studies:

- Adaptive remeshing.

A disadvantage of the implemented phase field model is that this model can only provide reasonable results if the length-scale parameter is chosen sufficiently small in conjunction with the mesh size. Thus, this model requires an extremely dense finite element discretization to resolve the continuum interpolation of the phase field accurately. This requirement is numerical demanding regarding computational time and memory. Hence, the implemented model should be extended to account for adaptive remeshing concepts in future studies, as suggested by Hofacker and Miehe [48].

- Higher-order degradation function.

Kuhn et al. [64] presented a cubic degradation function. They found that this function provided more accurate results than the quadratic function used in this study. Furthermore, Steinke and Kaliske [110] introduced an enhanced type of the degradation function which leads to a more brittle behavior of the phase field model. Thus, an investigation into the effect of higher-order degradation functions and the enhanced degradation function of Steinke and Kaliske [110] on the accuracy of results constitutes a suitable research undertaking. 
- Higher-order fracture functional.

This study used a second-order fracture energy functional. In contrast, Borden et al. [13] and [45] presented a fourth-order functional. These authors reported that higher-order fracture energy functionals provide better accuracy and convergence rates. Hence, future studies can be undertaken to validate these findings.

- Anisotropic split methods.

A comparison of the different anisotropic spit methods available in literature can constitute a suitable future work. In addition to the volumetric-deviatoric split of Amor et al. [5], the methods proposed by Miehe et al. [78, 79], Hesch et al. [45] and Steinke and Kaliske [110] can be considered for investigation.

- Algorithmic implementation method.

This study implemented the monolithic algorithmic scheme to solve the displacement and phase fields simultaneously. In the future, the staggered scheme can also be implemented and investigated. This scheme solves the displacement and phase fields successively, as presented in e.g., Borden et al. [12], Hofacker and Miehe [47], and Liu et al. [73]. Another scheme to be considered is the line search assisted monolithic approach proposed by Gerasimov and De Lorenzis [35]. The authors reported that their scheme provides a more robust implementation.

- Irreversibility approach.

Linse et al. [72] found that the irreversibility approached adopted in this study could lead to error in the results of simulations. They determined that this error may amount to up to $100 \%$. Hence, they questioned whether the irreversibility should be ensured in any way. They further suggested that the preferable approach is to fix the phase field value to one when a threshold value is attained. Thus, an investigation into the irreversibility model of the fracture process should be considered.

- Modeling of pre-existing cracks

This study modeled pre-existing cracks by incorporating a discrete crack in the geometry. Alternative approaches can also be considered in future studies. Schlüter et al. [101] proposed an explicit inclusion of the crack in the domain through of the use of the phase field. On the other hand, Borden [11] proposed the use of an initial strain-history field to model pre-existing cracks at any location of the domain. 
- Ductile and thermal fracture.

The implemented model can be extended to account for ductile and thermal fracture. Ductile fracture has already been investigated by Ambati and De Lorenzis [1], Kuhn and Noll [63] and Miehe et al. [80]. On thermal fracture, Schlüter et al. [102] presented qualitative results of dynamic thermal fracture in the context of irradiation damage.

- Additional inclusions to the implemented phase field model.

Several inclusions can be considered for the implemented model. For example, the inclusion of a shell-framework can be considered to address thin problems, as proposed by Reinoso et al. [97] and Kiendl et al. [55]. An anisotropic crack driving force could be included to model fracture in soft biological tissues as introduced by Raina and Miehe [94]. The implemented model can also be extended to fracture in rubbery polymers with the inclusion of a micromechanical modeling of bulk elasticity and failure as presented by Miehe and Schänzel [77]. Another extension could be the model developed by Zhou et al. [121] to address fracture in poroelastic media. Furthermore, Shanthraj et al. [105] proposed a finite-strain anisotropic method to model the localization of damage on a defined family of crystallographic planes, which is characteristic of cleavage fracture.

\subsubsection{Application of the phase field model to the fracture of Antarctic ice}

After the studies proposed above, the phase field model can then be used to address the fracture of Antarctic sea ice. In this regard, the following studies are proposed:

- Modeling of fracture in Antarctic sea ice.

Fracture in Antarctic sea ice can be brittle or ductile, subject to the loading rates, and thermal due to the seasonal change of temperature in spring and summer [46] [104]. Additionally, the ice can be considered as a porous material [114]. Thus, the phase field model for Antarctic ice should be able to address these different fracture types. Another important aspect of this proposed study is the identification of the adequate material model of the sea ice.

- Benchmarking and calibration of the phase field model.

This study involves the calibration of the phase field model to experimental sea ice data. The objective of this study is to model the Antarctic sea ice break-up on a regional scale during 
springtime. 


\section{Appendix A}

\section{SESKA}

SESKA is an in-house $\mathrm{C}++$ software package developed by A. Prof S. Skatulla with the purpose of solving continuum mechanics problems. This hybrid package uses both the finite element and element-free Galerkin methods for the calculation process, while the pre- and post- processing commercial $\mathrm{GiD}^{1}$ handles the geometry definition and solution visualization. The parallelization of SESKA processes using the OpenMPI library enables to solve large problems using high-performance computers. SESKA solves problems by first constructing shape functions and assembling the contribution of particles to the global stiffness, damping and mass matrices. Based on the problem description, SESKA then uses a linear or Newton-Raphson scheme to solve the set of equations [99].

\footnotetext{
${ }^{1}$ More details on the commercial software GiD are provided in https://www.gidhome.com.
} 


\section{Appendix B}

\section{Ethics approval}

EBE Faculty: Assesement of Ethics In Research Projects

Any persen pianning to undertake research in the Faculty of Englneering and the Bullt Environment at the Univereily of Cape Town is required to complate this form belore collociting or analyaing data. When completed it should be submittied to the supervisor (Where applicabile) and from there to the Hoad of Deparment. If any of the quastions bolow have been answered YS, and the appllcal

. compioted form with the disesertationithesis when it is submitted for exsmination.

Name of Princlpal Researcherlstudent:

EMMANUEL OMATUKU NGONGO

If a student: Degree:

MSC CIVL ENGINEERING
Department:

CIVIL ENGINEERING

Supervisor:

SEBASTIAN SKATULLLA

If a Research Contract indicate source of funding/eponsorehlp:

NONE

Research Project Titte:

MODELLING OF CRACKS USING THE PHASE FIELD METHOD. APPLICATIONS TO SEA ICE IN ANTARCTICA.

Overview of ethlcs issues in your research project:

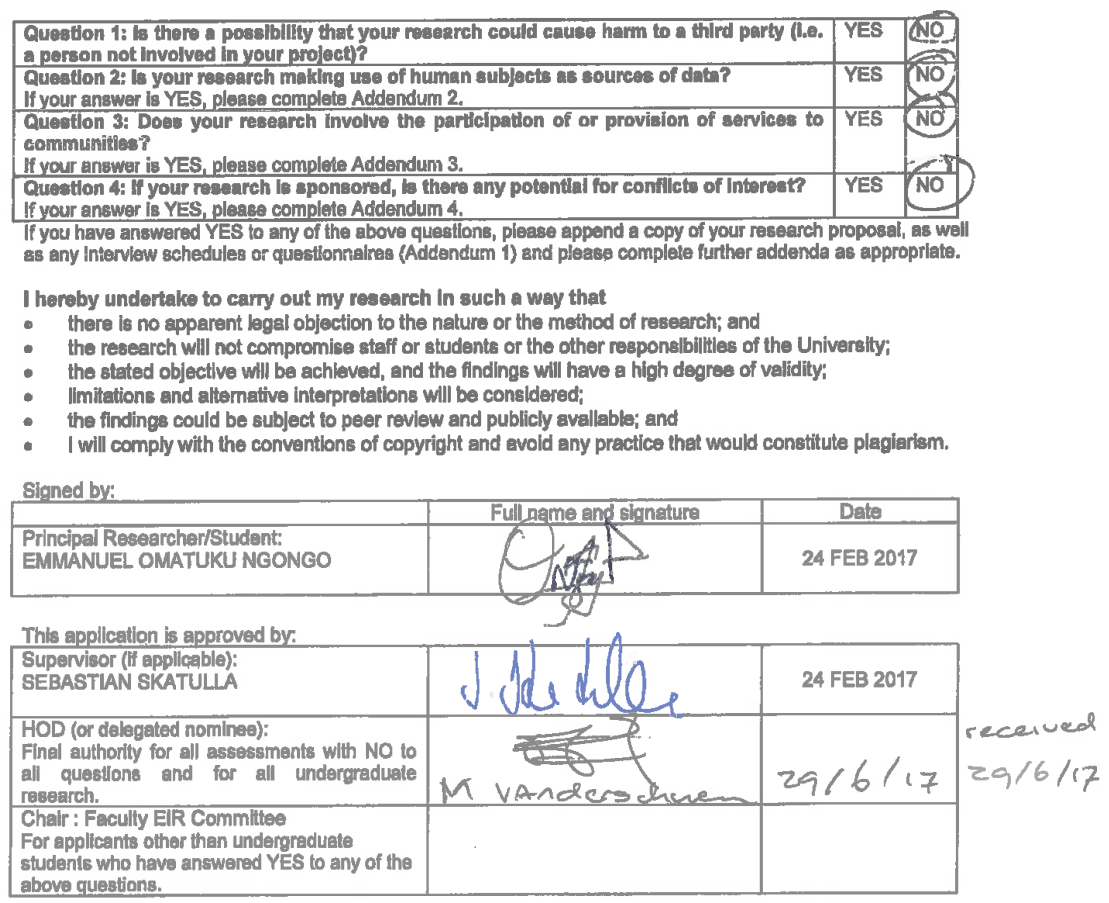




\section{Bibliography}

[1] M. Ambati and L. De Lorenzis. Phase-field modeling of brittle and ductile fracture in shells with isogeometric NURBS-based solid-shell elements, volume 312. Elsevier B.V., 2016. doi: 10.1016/j.cma.2016.02.017.

[2] M. Ambati, T. Gerasimov, and L. De Lorenzis. A review on phase-field models of brittle fracture and a new fast hybrid formulation, volume 57. 2014. ISBN 0178-7675. doi: 10.1007/ s00466-014-1109-y.

[3] M. Ambati, R. Kruse, and L. De Lorenzis. A phase-field model for ductile fracture at finite strains and its experimental verification. Computational Mechanics, 57(1):149-167, 2016. ISSN 01787675. doi: 10.1007/s00466-015-1225-3.

[4] L. Ambrosio and V. M. Tortorelli. Approximation of functional depending on jumps by elliptic functional via t-convergence. Communications on Pure and Applied Mathematics, 43(8):9991036, dec 1990. ISSN 00103640. doi: 10.1002/cpa.3160430805.

[5] H. Amor, J. J. Marigo, and C. Maurini. Regularized formulation of the variational brittle fracture with unilateral contact: Numerical experiments, volume 57. 2009. ISBN 0022-5096. doi: 10.1016/j.jmps.2009.04.011.

[6] I. S. Aranson, V. A. Kalatsky, and V. M. Vinokur. Continuum field description of crack propagation. Physical Review Letters, 85(1):118-121, 2000. ISSN 00319007. doi: 10.1103/ PhysRevLett.85.118.

[7] F. Armero and C. Linder. Numerical simulation of dynamic fracture using finite elements with embedded discontinuities. International Journal of Fracture, 160(2):119-141, 2009. ISSN 03769429. doi: 10.1007/s10704-009-9413-9. 
[8] T. Belytschko and T. Black. Elastic crack growth in finite elements with minimal remeshing. International Journal for Numerical Methods in Engineering, 45(July 1998):601-620, 1999. ISSN 0029-5981.

[9] T. Belytschko, H. Chen, J. Xu, and G. Zi. Dynamic crack propagation based on loss of hyperbolicity and a new discontinuous enrichment. International Journal for Numerical Methods in Engineering, 58(12):1873-1905, 2003. ISSN 00295981. doi: 10.1002/nme.941.

[10] J. Bonet and R. Wood. Nonlinear Continuum Mechanics for Finite Element Analysis (2nd Edition). 2008. ISBN 9780511755446 - 9780521838702. doi: 10.1017/CBO9780511755446.

[11] M. J. Borden. Isogeometric analysis of phase-field models for dynamic brittle and ductile fracture. Thesis, 2012.

[12] M. J. Borden, C. V. Verhoosel, M. A. Scott, T. J. R. Hughes, and C. M. Landis. A phase-field description of dynamic brittle fracture, volume 217-220. Elsevier B.V., 2012. ISBN 0045-7825. doi: $10.1016 /$ j.cma.2012.01.008.

[13] M. J. Borden, T. J. R. Hughes, C. M. Landis, C. V. Verhoosel, and R. M. J. Borden. A higherorder phase-field model for brittle fracture: Formulation and analysis within the isogeometric analysis framework by A higher-order phase-field model for brittle fracture : Formulation and analysis within the isogeome. Number July. 2013.

[14] B. Bourdin, G. A. Francfort, and J. J. Marigo. Numerical experiments in revisited brittle fracture, volume 48. 2000. ISBN 0022-5096. doi: 10.1016/S0022-5096(99)00028-9.

[15] B. Bourdin, G. A. Francfort, and J. J. Marigo. The variational approach to fracture. 2008. ISBN 9781402063947. doi: 10.1007/978-1-4020-6395-4.

[16] B. Bourdin, C. Maurini, and M. Knepley. Numerical Simulation of Reservoir Stimulation - a Variational. Thirty-Sixth Workshop on Geothermal reservior Engineering, 2011.

[17] G. Caginalp and J. Jones. A derivation and analysis of phase field models of thermal alloys, 1995. ISSN 1096035X.

[18] G. Caginalp and E. A. Socolovsky. Computation of sharp phase boundaries by spreading: The planar and spherically symmetric cases. Journal of Computational Physics, 95(1):85-100, 1991. ISSN 0021-9991. doi: https://doi.org/10.1016/0021-9991(91)90254-I. 
[19] J. W. Cahn and J. E. Hilliard. Free Energy of a Nonuniform System. I. Interfacial Free Energy. The Journal of Chemical Physics, 28(2):258-267, 1958.

[20] G. Camacho and M. Ortiz. Computational modelling of impact damage in brittle materials. International Journal of Solids and Structures, 33(20-22):2899-2938, aug 1996. ISSN 00207683. doi: 10.1016/0020-7683(95)00255-3.

[21] J. Carlsson and P. Isaksson. Dynamic crack propagation in wood fibre composites analysed by high speed photography and a dynamic phase field model. International Journal of Solids and Structures, apr 2018. ISSN 00207683. doi: 10.1016/j.ijsolstr.2018.04.015.

[22] A. Chambolle, G. a. Francfort, and J.-J. Marigo. Revisiting Energy Release Rates in Brittle Fracture. Journal of Nonlinear Science, 20(4):395-424, 2010. ISSN 0938-8974. doi: 10.1007/ s00332-010-9061-2.

[23] V. Chiaruttini, V. Riolo, and F. Feyel. Advanced remeshing techniques for complex 3D crack propagation. Icf13, pages 1-9, 2013.

[24] J. B. Collins and H. Levine. Diffuse interface model of diffusion-limited crystal growth. Phys. Rev. B, 31(9):6119-6122, may 1985. doi: 10.1103/PhysRevB.31.6119.

[25] F. Corson, M. Adda-Bedia, H. Henry, and E. Katzav. Thermal fracture as a framework for quasi-static crack propagation. International Journal of Fracture, 158(1):1-14, 2009. ISSN 03769429. doi: 10.1007/s10704-009-9361-4.

[26] D. Doyen, A. Ern, and S. Piperno. Time-Integration Schemes for the Finite Element Dynamic Signorini Problem. SIAM Journal on Scientific Computing, 33(1):223-249, 2011. ISSN 10648275. doi: $10.1137 / 100791440$.

[27] R. Duddu and H. Waisman. A nonlocal continuum damage mechanics approach to simulation of creep fracture in ice sheets, volume 51. 2013. doi: 10.1007/s00466-012-0778-7.

[28] L. O. Eastgate, J. P. Sethna, M. Rauscher, T. Cretegny, C. S. Chen, and C. R. Myers. Fracture in mode I using a conserved phase-field model. Physical Review E - Statistical Physics, Plasmas, Fluids, and Related Interdisciplinary Topics, 65(3):1-10, 2002. ISSN 1063651X. doi: 10.1103/PhysRevE.65.036117.

[29] B. Echebarria, R. Folch, A. Karma, and M. Plapp. Quantitative phase-field model of alloy solidification. Physical Review E - Statistical Physics, Plasmas, Fluids, and Related Interdisciplinary Topics, 70(6):22, 2004. ISSN 1063651X. doi: 10.1103/PhysRevE.70.061604. 
[30] F. Erdogan and G. C. Sih. On the Crack Extension in Plates Under Plane Loading and Transverse Shear. Journal of Basic Engineering, 85(4):519-525, 1963.

[31] M. Fagerström and R. Larsson. Theory and numerics for finite deformation fracture modelling using strong discontinuities. International Journal for Numerical Methods in Engineering, 66 (6):911-948, 2006. ISSN 00295981. doi: 10.1002/nme.1573.

[32] G. J. Fix. Phase field models for free boundary problems. Applied Math. Modelling., in press,, 1982

[33] G. Francfort and J.-J. Marigo. Revisiting brittle fracture as an energy minimization problem. Journal of the Mechanics and Physics of Solids, 46(8):1319-1342, 1998. ISSN 00225096. doi: 10.1016/S0022-5096(98)00034-9.

[34] G. Gaginalp, P. Fife, G. Caginalp, and P. Fife. Phase-field methods for interfacial boundaries. Physical Review B, 33(11):7792-7794, jun 1986. doi: 10.1021/j100783a023.

[35] T. Gerasimov and L. De Lorenzis. A line search assisted monolithic approach for phase-field computing of brittle fracture. Computer Methods in Applied Mechanics and Engineering, 312: 276-303, 2016. ISSN 00457825. doi: 10.1016/j.cma.2015.12.017.

[36] A. Giacomini. Ambrosio-Tortorelli approximation of quasi-static evolution of brittle fractures. Calculus of Variations and Partial Differential Equations, 22(2):129-172, feb 2005. ISSN 14320835. doi: 10.1007/s00526-004-0269-6.

[37] V. L. Ginzburg and L. D. Landau. On the Theory of superconductivity. Zh. Eksp. Teor. Fiz., 20:1064-1082, 1959 .

[38] R. V. Gol'dstein and R. L. Salganik. Brittle fracture of solids with arbitrary cracks. International Journal of Fracture, 10(4):507-523, 1974. ISSN 03769429. doi: 10.1007/BF00155254.

[39] A. A. Griffith. The Phenomena of Rupture and Flow in Solids, volume 221. 1921. ISBN 0264-3952. doi: 10.1098/rsta.1921.0006.

[40] D. Gross and T. Seelig. Fracture mechanics: with an introduction to micromechanics. Springer,, Berlin, 2010. ISBN 9780387263960. doi: 10.1007/b22134.

[41] E. Gürses and C. Miehe. A computational framework of three-dimensional configurationalforce-driven brittle crack propagation. Computer Methods in Applied Mechanics and Engineering, 198(15-16):1413-1428, mar 2009. ISSN 0045-7825. doi: 10.1016/J.CMA.2008.12.028. 
[42] V. Hakim and A. Karma. Crack Path Prediction in Anisotropic Brittle Materials. Phys. Rev. Lett., 95(23):235501, dec 2005. doi: 10.1103/PhysRevLett.95.235501.

[43] H. Henry and H. Levine. Dynamic Instabilities of Fracture under Biaxial Strain Using a Phase Field Model. Phys. Rev. Lett., 93(10):105504, sep 2004. doi: 10.1103/PhysRevLett.93.105504.

[44] C. Hesch and K. Weinberg. Thermodynamically consistent algorithms for a finite-deformation phase-field approach to fracture. International Journal for Numerical Methods in Engineering, 99:906-924, 2014. ISSN 0743-1619. doi: 10.1002/nme.

[45] C. Hesch, A. Gil, R. Ortigosa, M. Dittmann, C. Bilgen, P. Betsch, M. Franke, A. Janz, and K. Weinberg. A framework for polyconvex large strain phase-field methods to fracture. Computer Methods in Applied Mechanics and Engineering, 317:649-683, apr 2017. ISSN 00457825. doi: 10.1016/J.CMA.2016.12.035.

[46] W. D. Hibler. Sea ice fracturing on the large scale, volume 68. 2001. doi: 10.1016/ S0013-7944(01)00035-2.

[47] M. Hofacker and C. Miehe. Continuum phase field modeling of dynamic fracture: Variational principles and staggered FE implementation, volume 178. 2012. ISBN 0376-9429. doi: 10. 1007/s10704-012-9753-8.

[48] M. Hofacker and C. Miehe. A phase field model of dynamic fracture: Robust field updates for the analysis of complex crack patterns. Number 93. 2013. ISBN 978-1-4577-0079-8. doi: $10.1002 /$ nme.

[49] G. A. Holzapfel. Nonlinear Solid Mechanics: A Continuum Approach for Engineering. John Wiley \& Sons, Ltd, 2000.

[50] T. J. R. Hughes. The Finite Element Method: Linear Static and Dynamic Finite Element Analysis (Dover Civil and Mechanical Engineering). Dover Publications, aug 2000. ISBN 0486411818 .

[51] G. R. Irwin. Analysis of Stresses and Strains Near the End of a Crack Traversing a Plate. J. Appl. Mech., 1957.

[52] A. Karma and A. E. Lobkovsky. Unsteady Crack Motion and Branching in a Phase-Field Model of Brittle Fracture. Phys. Rev. Lett., 92(24):245510, jun 2004. doi: 10.1103/PhysRevLett.92. 245510. 
[53] A. Karma and W.-J. Rappel. Quantitative phase-field modeling of dendritic growth in two and three dimensions. Phys. Rev. E, 57(4):4323-4349, apr 1998. doi: 10.1103/PhysRevE.57.4323.

[54] A. Karma, D. A. Kessler, and H. Levine. Phase-Field Model of Mode III Dynamic Fracture. Phys. Rev. Lett., 87(4):45501, jul 2001. doi: 10.1103/PhysRevLett.87.045501.

[55] J. Kiendl, M. Ambati, L. De Lorenzis, H. Gomez, and A. Reali. Phase-field description of brittle fracture in plates and shells. Computer Methods in Applied Mechanics and Engineering, 312: 374-394, 2016. ISSN 00457825. doi: 10.1016/j.cma.2016.09.011.

[56] R. Kobayashi. Modeling and numerical simulations of dendritic crystal growth. Physica D: Nonlinear Phenomena, 63(3-4):410-423, mar 1993. ISSN 0167-2789. doi: 10.1016/ 0167-2789(93)90120-P.

[57] R. Kobayashi. Phase Field Simulations of Dendritic Solidification. Advanced Materials '93, pages 529-532, 1994. doi: 10.1016/B978-0-444-81993-2.50128-1.

[58] R. Kobayashi. A Numerical Approach to Three-Dimensional Dendritic Solidification. Experimental Mathematics, 3(1):59-81, 1994. doi: 10.1080/10586458.1994.10504577.

[59] R. Kobayashi and J. A. Warren. Modeling the formation and dynamics of polycrystals in 3D. Physica A: Statistical Mechanics and its Applications, 356(1):127-132, 2005. ISSN 03784371. doi: 10.1016/j.physa.2005.05.024.

[60] C. Koenke, R. Harte, W. Krätzig, and O. Rosenstein. On adaptive remeshing techniques for crack simulation problems. Engineering Computations, 15(1):74-88, feb 1998. ISSN 0264-4401. doi: 10.1108/02644409810200695.

[61] C. Kuhn. Numerical and Analytical Investigation of a Phase Field Model for Fracture. 2013. ISBN 9783942695060.

[62] C. Kuhn and R. Müller. A continuum phase field model for fracture, volume 77. 2010.

[63] C. Kuhn and T. Noll. On phase field modeling of ductile fracture, volume 54. 2016. doi: 10.1002/gamm.201610003.

[64] C. Kuhn, A. Schlüter, and R. Müller. On degradation functions in phase field fracture models, volume 108. Elsevier B.V., 2015. doi: 10.1016/j.commatsci.2015.05.034.

[65] L. D. Landau and E. M. Lifshitz. Statistical Physics, 3rd. Edition (Part 1 Oxford: Pergamon Press. 1980), 1980. 
[66] J. S. Langer. Models of pattern formation in first-order phase transitions, pages 165-186. 1986. doi: 10.1142/9789814415309_0005.

[67] C. J. Larsen. Models for Dynamic Fracture Based on Griffith's Criterion. In IUTAM Symposium on Variational Concepts with Applications to the Mechanics of Materials, volume 21, pages 131-140. Springer Netherlands, 2010. ISBN 978-90-481-9194-9. doi: 10.1007/ 978-90-481-9195-6.

[68] C. J. Larsen, C. Orton, and E. Süli. Existence of solutions to a regularized model of dynamic fracture. Mathematical Models and Methods in Applied Sciences, 20(07):1021-1048, 2010. doi: $10.1142 / \mathrm{S} 0218202510004520$.

[69] D. Leguillon. Strength or toughness? A criterion for crack onset at a notch. European Journal of Mechanics, A/Solids, 21(1):61-72, 2002. ISSN 09977538. doi: 10.1016/S0997-7538(01) 01184-6.

[70] J. Li and X. B. Zhang. A criterion study for non-singular stress concentrations in brittle or quasi-brittle materials. Engineering Fracture Mechanics, 73(4):505-523, 2006. ISSN 00137944. doi: $10.1016 /$ j.engfracmech.2005.09.001.

[71] C. Linder and F. Armero. Finite Elements with Embedded Branching. Finite Elem. Anal. Des., 45(4):280-293, mar 2009. ISSN 0168-874X. doi: 10.1016/j.finel.2008.10.012.

[72] T. Linse, P. Hennig, M. Kästner, and R. de Borst. A convergence study of phase-field models for brittle fracture. Engineering Fracture Mechanics, 184(September):307-318, 2017. ISSN 00137944. doi: 10.1016/j.engfracmech.2017.09.013.

[73] G. Liu, Q. Li, M. A. Msekh, and Z. Zuo. Abaqus implementation of monolithic and staggered schemes for quasi-static and dynamic fracture phase-field model, volume 121. Elsevier B.V., 2016. doi: 10.1016/j.commatsci.2016.04.009.

[74] D. Logan. A first course in the finite element method. 2012. ISBN 9780495668251.

[75] J. M. Melenk and I. Babuška. The partition of unity finite element method: Basic theory and applications. Computer Methods in Applied Mechanics and Engineering, 139(1-4):289-314, 1996. ISSN 0374-2830.

[76] C. Miehe and E. Gürses. A robust algorithm for configurational-force-driven brittle crack propagation with R-adaptive mesh alignment. International Journal for Numerical Methods in Engineering, 72:127-155, 2007. ISSN 0743-1619. doi: 10.1002/nme. 
[77] C. Miehe and L. M. Schänzel. Phase field modeling of fracture in rubbery polymers. Part I: Finite elasticity coupled with brittle failure. Journal of the Mechanics and Physics of Solids, 65(1):93-113, 2014. ISSN 00225096. doi: 10.1016/j.jmps.2013.06.007.

[78] C. Miehe, M. Hofacker, and F. Welschinger. A phase field model for rate-independent crack propagation: Robust algorithmic implementation based on operator splits, volume 199. Elsevier B.V., 2010. ISBN 0045-7825. doi: 10.1016/j.cma.2010.04.011.

[79] C. Miehe, F. Welschinger, and M. Hofacker. Thermodynamically consistent phase-field models of fracture: Variational principles and multi-field FE implementations. Number 83. 2010. ISBN 978-1-4577-0079-8. doi: 10.1002/nme.

[80] C. Miehe, F. Aldakheel, and A. Raina. Phase field modeling of ductile fracture at finite strains: A variational gradient-extended plasticity-damage theory. International Journal of Plasticity, 84:1-32, 2016. ISSN 07496419. doi: 10.1016/j.ijplas.2016.04.011.

[81] C. Miehe, F. Aldakheel, and S. Teichtmeister. Phase-field modeling of ductile fracture at finite strains: A robust variational-based numerical implementation of a gradient-extended theory by micromorphic regularization. International Journal for Numerical Methods in Engineering, 111(9):816-863, 2017. ISSN 10970207. doi: 10.1002/nme.5484.

[82] N. Moës and T. Belytschko. Extended finite element method for cohesive crack growth. Engineering Fracture Mechanics, 69(7):813-833, 2002. ISSN 00137944. doi: 10.1016/ S0013-7944(01)00128-X.

[83] N. Moës, J. Dolbow, and T. Belytschko. A finite element method for crack growth without remeshing. 150(February):131-150, 1999.

[84] S. Mohammadi. Extended Finite Element Method. Blackwell Publishing Ltd, Tehran, 2008. ISBN 1405170603. doi: 10.1002/9780470697795.

[85] R. Müller, D. Gross, D. Schrade, and B. X. Xu. Phase field simulation of domain structures in ferroelectric materials within the context of inhomogeneity evolution. International Journal of Fracture, 147(1-4):173-180, 2007. ISSN 03769429. doi: 10.1007/s10704-007-9153-7.

[86] D. Mumford and J. Shah. Optimal approximations by piecewise smooth functions and associated variational problems. Communications on Pure and Applied Mathematics, 42(5):577-685, 1989. ISSN 10970312. doi: 10.1002/cpa.3160420503. 
[87] N. M. Newmark. A method of computation for structural dynamics. American Society of Civil Engineers, 1959.

[88] T. T. Nguyen, J. Yvonnet, M. Bornert, C. Chateau, K. Sab, R. Romani, and R. Le Roy. On the choice of parameters in the phase field method for simulating crack initiation with experimental validation. International Journal of Fracture, 2016. ISSN 15732673. doi: 10. 1007/s10704-016-0082-1.

[89] M. Paggi and J. Reinoso. Revisiting the problem of a crack impinging on an interface: A modeling framework for the interaction between the phase field approach for brittle fracture and the interface cohesive zone model. Computer Methods in Applied Mechanics and Engineering, 321:145-172, 2017. ISSN 00457825. doi: 10.1016/j.cma.2017.04.004.

[90] A. Pandolfi and M. Ortiz. An Efficient Adaptive Procedure for Three-Dimensional Fragmentation Simulations. Engineering with Computers, 18(2):148-159, aug 2002. ISSN 1435-5663. doi: $10.1007 / \mathrm{s} 003660200013$.

[91] O. Penrose and P. C. Fife. Thermodynamically consistent models of phase-field type for the kinetic of phase transitions. Physica D: Nonlinear Phenomena, 43(1):44-62, may 1990. ISSN 0167-2789. doi: 10.1016/0167-2789(90)90015-H.

[92] O. Penrose and P. C. Fife. On the relation between the standard phase-field model and a "thermodynamically consistent" phase-field model. Physica D: Nonlinear Phenomena, 69 (1-2):107-113, nov 1993. ISSN 0167-2789. doi: 10.1016/0167-2789(93)90183-2.

[93] M. Pundir. Masters Thesis: Crack propagation analysis using Phase-Field Model in the framework of Isogeometric Analysis and Finite Cell Method. Master of science, Delft University of Technology, 2016.

[94] A. Raina and C. Miehe. A phase-field model for fracture in biological tissues. Biomechanics and Modeling in Mechanobiology, 2016.

[95] P. Rampal, J. Weiss, C. Dubois, and J. M. Campin. IPCC climate models do not capture Arctic sea ice drift acceleration: Consequences in terms of projected sea ice thinning and decline, volume 116. 2011. ISBN 0148-0227. doi: 10.1029/2011JC007110.

[96] J. N. Reddy. An Introduction to Continuum Mechanics. 2013. ISBN 9781107025431. 
[97] J. Reinoso, M. Paggi, and C. Linder. Phase field modeling of brittle fracture for enhanced assumed strain shells at large deformations: formulation and finite element implementation. Computational Mechanics, 59(6):981-1001, 2017. ISSN 01787675. doi: 10.1007/s00466-017-1386-3.

[98] J. Reinoso, M. Paggi, and C. Linder. Phase field modeling of brittle fracture for enhanced assumed strain shells at large deformations: formulation and finite element implementation. Computational Mechanics, 59(6):981-1001, 2017. ISSN 01787675. doi: 10.1007/s00466-017-1386-3.

[99] K. Sack. Biological tissue mechanics with fibres modelled as one dimensional Cosserat continua. Applications to cardiac tissue in healthy and diseased states. Masters, University of Cape Town, 2014.

[100] C. Sansour, W. Wagner, P. Wriggers, and J. Sansour. An energy-momentum integration scheme and enhanced strain finite elements for the non-linear dynamics of shells. International Journal of Non-Linear Mechanics, 37(4):951-966, 2002. ISSN 0020-7462. doi: https://doi.org/ 10.1016/S0020-7462(01)00108-1.

[101] A. Schlüter, A. Willenbücher, C. Kuhn, and R. Müller. Phase field approximation of dynamic brittle fracture, volume 54. 2014. ISBN 0178-7675. doi: 10.1007/s00466-014-1045-x.

[102] A. Schlüter, C. Kuhn, R. Müller, M. Tomut, C. Trautmann, H. Weick, and C. Plate. Phase field modelling of dynamic thermal fracture in the context of irradiation damage. Continuum Mechanics and Thermodynamics, 29(4):977-988, 2017. ISSN 09351175. doi: 10.1007/s00161-015-0456-z.

[103] D. Schrade, R. Mueller, D. Gross, T. Utschig, V. Y. Shur, and D. C. Lupascu. Interaction of domain walls with defects in ferroelectric materials. Mechanics of Materials, 39(2):161-174, 2007. ISSN 01676636. doi: 10.1016/j.mechmat.2006.04.002.

[104] E. M. Schulson and P. Duval. Creep and Fracture of Ice. 2009. ISBN 0521806208. doi: 10.1017/CBO9780511581397.

[105] P. Shanthraj, B. Svendsen, L. Sharma, F. Roters, and D. Raabe. Elasto-viscoplastic phase field modelling of anisotropic cleavage fracture, volume 99. Elsevier, 2017. doi: 10.1016/j. jmps.2016.10.012. 
[106] J.-H. Song and T. Belytschko. Geometric numerical integration for complex dynamics of tethered spacecraft. International Journal for Numerical Methods in Engineering, 77:360-385, 2009. ISSN 0743-1619. doi: 10.1002/nme.

[107] J. H. Song, P. M. Areias, and T. Belytschko. A method for dynamic crack and shear band propagation with phantom nodes. International Journal for Numerical Methods in Engineering, 67(6):868-893, 2006. ISSN 00295981. doi: 10.1002/nme.1652.

[108] J.-H. Song, H. Wang, and T. Belytschko. A comparative study on finite element methods for dynamic fracture. Computational Mechanics, 42(2):239-250, 2008. ISSN 0178-7675. doi: 10.1007/s00466-007-0210-x.

[109] R. Spatschek, E. Brener, and A. Karma. Phase field modeling of crack propagation. Philosophical Magazine, 91(1):75-95, 2011. doi: 10.1080/14786431003773015.

[110] C. Steinke and M. Kaliske. A phase-field crack model based on directional stress decomposition. Computational Mechanics, 2018. ISSN 0178-7675. doi: 10.1007/s00466-018-1635-0.

[111] M. Strobl and T. Seelig. A novel treatment of crack boundary conditions in phase field models of fracture. Pamm, 15(1):155-156, 2015. ISSN 16177061. doi: 10.1002/pamm.201510068.

[112] E. B. Tadmor, R. F. Miller, and R. S. Elliott. Continuum Mechanics and Thermodynamics. 2012. ISBN 9781107008267. doi: 10.1017/CBO9781139017657.

[113] S. Teichtmeister, D. Kienle, F. Aldakheel, and M. A. Keip. Phase field modeling of fracture in anisotropic brittle solids. International Journal of Non-Linear Mechanics, 97(June):1-21, 2017. ISSN 00207462. doi: 10.1016/j.ijnonlinmec.2017.06.018.

[114] D. N. Thomas and G. S. Dieckmann. Sea Ice. Wiley-Blackwell, 2010. ISBN 9781405185806. doi: $10.1002 / 9780470757161$.

[115] M. Thomas. Analysis and Simulations for a Phase Field Fracture Model at Finite Strains. pages $1-25,2017$.

[116] C. V. Verhoosel and R. de Borst. A phase-field model for cohesive fracture. International Journal for Numerical Methods in Engineering, 96:43-62, 2013. ISSN 0743-1619. doi: 10. $1002 /$ nme. 
[117] K. Weinberg and C. Hesch. A high-order finite deformation phase-field approach to fracture. Continuum Mechanics and Thermodynamics, 29(4):935-945, 2017. ISSN 09351175. doi: 10. 1007/s00161-015-0440-7.

[118] P. D. P. Wriggers. Nonlinear Finite Element Methods. 2001. ISBN 9783540710004. doi: 10.1007/978-3-540-71001-1.

[119] C. H. Wu. Maximum-energy-release-rate criterion applied to a tension-compression specimen with crack. Journal of Elasticity, 8(3):235-257, 1978. ISSN 15732681. doi: 10.1007/ BF00130464.

[120] X.-P. Xu and A. Needleman. Numerical simulations of fast crack growth in brittle solids. Journal of the Mechanics and Physics of Solids, 42(9):1397-1434, sep 1994. ISSN 0022-5096. doi: 10.1016/0022-5096(94)90003-5.

[121] S. Zhou, X. Zhuang, and T. Rabczuk. A phase-field modeling approach of fracture propagation in poroelastic media. Engineering Geology, pages 1-18, 2018. ISSN 0013-7952. doi: 10.1016/ J.ENGGEO.2018.04.008.

[122] O. C. Zienkiewicz. The Finite Element Method for Solid and Structural Mechanics. 2000.

[123] O. C. Zienkiewicz, R. L. Taylor, and D. Fox. The Finite Element Method for Solid and Structural Mechanics. 2014. ISBN 9781856176347. doi: 10.1016/B978-1-85617-634-7.00007-7. 